\title{
Development of the Galaxy Chronic Obstructive Pulmonary Disease (COPD) Model Using Data from ECLIPSE: Internal Validation of a Linked Equations Cohort Model
}

Andrew Briggs, DPhil*, Timothy Baker, BSc, Nancy A. Risebrough, BA, Mike Chambers, MSc, Sebastian Gonzalez-McQuire, MSc ${ }^{\dagger}$, Afisi S. Ismaila, PhD, Alex Exuzides, PhD, Chris Colby, PhD, Maggie Tabberer, MSc, Hana Muellerova, PhD, Nicholas Locantore, PhD, Maureen P.M.H. Rutten van-Mölken, PhD, David A. Lomas, PhD FRCP

Institute of Health and Wellbeing, University of Glasgow, Glasgow, United Kingdom (AB); ICON Plc, Morristown, NJ, USA (AB, TB); ICON Plc, Toronto, ON, Canada (NAR); Global Market Access and Healthcare Solutions, GSK, Brentford, United Kingdom (MC); Value Evidence and Outcomes, GSK R\&D, Uxbridge, United Kingdom (SGM, MT); Value Evidence and Outcomes, GSK R\&D, Research Triangle Park, NC, USA (ASI, NL); Clinical Epidemiology and Biostatistics, McMaster University, Hamilton, ON, Canada (ASI); ICON Plc, San Francisco, CA, USA (AE, CC); Worldwide Epidemiology, GSK R\&D, Uxbridge, United Kingdom (HM); Institute for Medical Technology Assessment, Erasmus University, Rotterdam, The Netherlands (MRvM); University College London, London, United Kingdom (DAL)

†Was employed by GSK at the time the research was conducted (current affiliation: Health Economics, Amgen, Zug, Switzerland)

*Address correspondence to:

Andrew H Briggs

William R Lindsay Chair of Health Economics, Health Economics \& Health Technology Assessment, Institute of Health \& Wellbeing, University of Glasgow

1 Lilybank Gardens, Glasgow, G12 8RZ, United Kingdom

Tel: +44 (0)141330 5017; Fax: +44(0)141330 5018

Email: andrew.briggs@glasgow.ac.uk

Funding: This study was funded by GSK, study number GHO-11-5102. 
This work has been published in abstract form, and was presented at the European International

Pharmacoeconomics and Outcomes Research Annual Congress, Amsterdam, 2014.

Keywords: COPD, QALY, cost, risk, model

\section{Running title: A Linked Equations Cohort Model in COPD}

Abbreviated title: Development of a COPD Model: Validation of a Linked Equations Cohort Model

\section{Word count: 3320}

Financial support for this study was provided entirely by a contract with GSK. The funding agreement ensured the authors' independence in designing the study, interpreting the data, writing, and publishing the report. The following authors are employed by the sponsor: MC, MT, ASI, NL, HM; SGM is a former employee of the sponsor. 


\section{ABSTRACT}

Background. The recent ISPOR-SMDM Modeling Good Research Practices Task Force on Modeling emphasized the importance of conceptualizing and validating models. We report a new model of chronic obstructive pulmonary disease (COPD) that was part of the Galaxy project. It is founded on a conceptual model, implemented using a novel linked-equation approach, and internally validated.

Methods. An expert panel developed a conceptual model including causal relationships between disease attributes, progression, and final outcomes. Risk equations describing these relationships were estimated using data from the Evaluation of COPD Longitudinally to Identify Predictive Surrogate Endpoints (ECLIPSE) study, with costs estimated using data from the TOwards a Revolution in COPD Health (TORCH) study. Implementation as a linked-equation model enabled direct estimation of health service costs and quality adjusted life-years (QALY) for COPD patients over their lifetimes. Internal validation compared three years of predicted cohort experience with ECLIPSE results.

Results. At three years, the Galaxy COPD model predicted annual exacerbation rate, and annual decline in forced expiratory volume in one second, all fell within the ECLIPSE data confidence limits, although overall survival was outside the observed confidence limits at three years. Projections of the risk equations over time permitted extrapolation to patient lifetimes. Averaging the predicted cost and QALY outcomes for the different patients within the ECLIPSE cohort gives an estimated lifetime cost of $£ 25,214$ (undiscounted)/£20,318 (discounted) and lifetime QALY of 6.45 (undiscounted/5.24 (discounted) per patient in ECLIPSE.

Conclusions. A new form of model for COPD was conceptualized, implemented, and internally validated, based on a series of linked equations using epidemiological data from ECLIPSE and cost data from TORCH. This Galaxy model may predict COPD outcomes from treatment effects on disease attributes such as lung function, exacerbations, symptoms or exercise capacity; however, further external validation is required. 


\section{INTRODUCTION}

Chronic obstructive pulmonary disease (COPD) is a respiratory disorder characterized by airflow obstruction, leading to shortness of breath, acute exacerbations, and death [1]. Globally, COPD is the fourth leading cause of death [2]. In England, more than 860,000 individuals were diagnosed with COPD in 20092010, corresponding to a prevalence of $1.6 \%$ [3]. However, it is estimated that most cases remain undiagnosed and that the true prevalence of COPD is much higher, potentially affecting more than 3 million people [1]. Smoking is the primary risk factor for developing COPD, and exposure to noxious fumes, dust, and gas are also risk factors [4]. The economic burden of COPD is high; it is one of the most costly inpatient conditions treated by the National Health Service in the United Kingdom, with an estimated direct cost of £810-930 million per year [1].

A number of models of COPD exist, which have been used for economic analysis for Health Technology Assessment (HTA). The majority of these are structured around disease 'states' that are usually defined using threshold values of $\%$ predicted forced expiratory volume in one second $\left(\mathrm{FEV}_{1}\right)$, adjusted for patients according to age, gender, and height [5,6]. However, recent prognostic modeling has shown that other aspects of the disease, such as symptoms and exercise capacity, are also important factors for survival [7-9], and clinical guidelines are beginning to acknowledge the multifaceted nature of COPD [2], such that there is inherent heterogeneity in health outcomes for COPD patients that depends on more than just $\mathrm{FEV}_{1}[8]$.

The Galaxy project was undertaken to develop a new disease/economic model for COPD, accounting for recent developments in the understanding of COPD as a multifaceted disease. The aim was to develop a model with sufficient flexibility to reflect different patient groups, based on an understanding of relationships between disease characteristics, risk factors, biomarkers, and surrogates, on relevant clinical outcomes and disease progression over time. This allows for a better capture of the inherent heterogeneity and corresponding adjustments made for different COPD patient populations, which facilitates comparisons of disease progression between different COPD subgroups. The development of the new Galaxy COPD model has adhered as closely as possible to best practice guidelines for medical decision modeling [10], which emphasize the importance of conceptual modeling [11]. The modeling and analysis we report in this paper was founded upon a conceptual model designed by a panel of experts prior to data analysis [12] and a series of linked risk equations that were 
developed from the Evaluation of COPD Longitudinally to Identify Predictive Surrogate Endpoints (ECLIPSE) data-set based on the relationships from the conceptual modeling exercise [13]. This paper brings the previous work together in a cohesive whole and internally validates the model using the same data that were used to generate model parameters. The model will also enable estimation of the cost effectiveness of a wide range of treatment interventions, acting through different mechanisms, used for the management of COPD.

\section{METHODS}

\section{Data sources}

The ECLIPSE study was a non-interventional, observational, multicenter, three-year study designed to determine mechanisms of disease progression and identify biomarkers that could serve as endpoints to measure progression of COPD [14]. This study included 2164 COPD patients, with an average age of 63.4 years, an average of 0.9 exacerbations in the year prior to study entry, and baseline \% predicted $\mathrm{FEV}_{1}$ of $48.3 \%$. Patients for whom full information on baseline characteristics were available $(n=1957)$ were included in the present model.

The TOwards a Revolution in COPD Health (TORCH) study, was a randomized, double-blind study ( $n$ =6112) comparing the effectiveness of salmeterol alone, fluticasone proprionate alone, a combination of salmeterol and fluticasone proprionate, and placebo, over a three-year period [15]. At baseline, the average age was 65.0 years; patients had an average of 1.0 exacerbation in the year prior to study entry, and baseline $\%$ predicted $\mathrm{FEV}_{1}$ was $44.1 \%$.

Patients included in the model had baseline characteristics that were slightly different from the original published studies, due to small amounts of missing information in the baseline covariates used in the modeling. However, there were no important differences between characteristics of the cohorts analyzed here to the original studies. Summary information for the analysis cohorts is given in the supplementary appendix in Table A1. 


\section{Model validation}

The conceptualization of the new model of COPD disease progression was reported separately [12], and led to the development of a conceptual model of COPD prognosis. Internal validation of the outputs predicted by the model was conducted by comparing them with published estimates of decline in reported lung function (measured as $\mathrm{FEV}_{1}$ ) and exercise capacity (measured as six-minute walking distance [6MWD]) within the ECLIPSE dataset. In addition, we compared the predicted exacerbation count, survival, and HRQoL with the observed data during each of the three years of ECLIPSE. ECLIPSE data were presented as point estimates with associated $95 \%$ confidence intervals (CIs) at the relevant time points. Estimated covariance matrices are reported in Appendix B (moderate exacerbations, fixed effects [Table A8]; severe exacerbations, fixed effects [Table A9]; $\mathrm{FEV}_{1}$, fixed effects [Table A10]; dyspnea, most versus none or several days, fixed effects [Table A11]; dyspnea (none vs most or several days), fixed effects [Table A12]; cough and sputum, fixed effects [Table A13]; 6MWD, fixed effects [Table A14]; SGRQ, fixed effects [Table A15]; mortality, fixed effects [Table A16]; survival, fixed effects [Table A17]; probability of hospitalization [Table A18]; ward days [Table A19]; intensive care unit [Table A20]; emergency room visits [Table A21]; days and nights at home [Table A22]; office visits [Table A13]; outpatient visits [Table A24]).

\section{Structure of the model}

The model described the important prognostic factors for COPD disease progression at baseline; the disease progression attributes that evolve over the lifetime of the model, such as exacerbations, lung function, exercise capacity, and symptoms; and the final outcomes of mortality, health-related quality of life (HRQoL), and health service resource use. In particular, lung function and exacerbations were assumed to affect each other in the conceptual model, with lung function affecting symptoms, symptoms affecting exacerbations and all three of these attributes affecting exercise capacity [12]. These relationships from the final conceptual model development were used as the basis for estimating a series of linked statistical equations based on data from ECLIPSE, which formed the epidemiological foundation of the disease model [13]. 
The statistical equations were brought together to estimate the course of COPD for a patient cohort in terms of the disease progression attributes defined in the conceptual model, through to final health outcomes of (quality adjusted) life expectancy, and health service cost. Observational data from ECLIPSE were used to estimate associations between disease progression attributes, together with mortality and HRQoL (measured by the St George's Respiratory Questionnaire for COPD [SGRQ-C]) [16], while adjusting for prognostic baseline covariates. However, because ECLIPSE did not record health service resource use, the resource use equations were estimated using data from TORCH.

Figure 1a illustrates the links between the statistical risk equations from the baseline prognostic variables through to the disease progression attributes of COPD [13] representing the relationships described in the conceptual model [12] above. Figure 1b shows the associations from the baseline covariates and the disease progression attributes through to the final health outcomes.

The Galaxy COPD model starts with the baseline covariates recorded in ECLIPSE (Appendix Table A1) and then builds on these by predicting how the baseline covariates affect the disease progression attributes of exacerbations, lung function, exercise capacity, and symptoms). Not all baseline variables were used in every equation, though all variables from Table A1 appeared in at least one of the equations. The baseline variables and longitudinal disease progression attributes are then used together to predict the final health outcomes of HRQoL/utility, survival, and health service resource use and cost over lifetime.

Health service resource use and cost. Compared with previously reported statistical analyses [13], the model represented in Figures 1a and $1 \mathrm{~b}$ includes health service resource use added to the list of final health outcomes. COPD-related health service resource use was based on an analysis of the TORCH data from the following health service resource use categories: hospital bed days (general ward, intensive care unit), emergency room visits, and outpatient visits (hospital, physician office, home day/night). Negative binomial count data models with a log link were used for the resource counts, adjusted for the baseline variables from the TORCH study, and are reported in full in Appendix A. Indicator variables for the treatment arm of the TORCH study were not used, so the intercept of the models (along with the rest of the coefficients) effectively represents a pooling across all treatment arms of TORCH. 
Weighting-predicted health service resource use in each category computed using the relevant United Kingdom unit costs for that category [17-19] enabled the annual health service costs associated with the progression of COPD to be calculated, taking into account the baseline factors as well as the central disease attributes on costs. These costs were then discounted at an annual rate of 3.5\% [20] to provide the net present value of COPD costs, predicted over the lifetime of each patient who enters the model.

Mapping SGRQ score to EuroQol-5 dimension (EQ-5D) utility. The previously reported statistical equations predict the patient-reported outcome of SGRQ-C, as collected in ECLIPSE [13]. However, for health economic modeling, a cardinal utility measure of HRQoL, such as the EQ-5D, is required as a basis for estimating quality-adjusted life years (QALYs). This was achieved firstly by converting SGRQ-C score to SGRQ Total score, as described previously [13], based on guidance from the user manual [15]. A mapping function, estimated from data available in TORCH where both instruments had been collected, suggested the following relationship between EQ-5D and SGRQ Total score [21]:

$$
\text { EQ-5D }=0.9617-0.0013 \times \text { SGRQ }-0.0001 \times \mathrm{SGRQ}^{2}+0.0231 \times \text { male }
$$

The longitudinal survival, lung function, exacerbations, symptoms and exercise capacity equations included a time trend term in the regression models [13]. These equations were projected from the observed three years of the ECLIPSE study to estimate lifetime outcomes. Weighting-predicted survival each year by predicted EQ-5D gave a yearly estimate of quality-adjusted survival. Summing this over the lifetime of the model, and applying a discount rate of 3.5\% [20] gave the net present value of estimated QALYs over the lifetime of each patient entering the model.

\section{Cohort estimation and baseline values}

In order to generate predicted values for the ECLIPSE cohort (employing the statistical equations reported previously [13] and the resource use equations described above and reported in the Appendix) the baseline characteristics of each of the 1957 patients forming the estimation sample of ECLIPSE were entered into the model. Not all equations include all baseline characteristics as covariates, but the set of baseline characteristics reported indicate that they were important predictors of outcome in at least one of the statistical 
equations (see [13] for full details). The resulting predictions of lifetime health service resource use cost and QALYs for each patient were estimated based on the predicted symptoms, exacerbations, lung function, and exercise capacity as presented in Figures $1 \mathrm{~A}$ and B. The distribution of costs and QALYs gave an indication of the heterogeneity of outcomes predicted by the model, for cohort members with different characteristics. Averaging these estimates gave the predicted average lifetime costs and QALYs for the entire ECLIPSE cohort. There has been much interest in the role of past exacerbations as a predictor of future exacerbations [22]. Therefore the importance of exacerbation history in the model is also explored as a prognostic variable.

\section{Probabilistic sensitivity analysis}

Uncertainty in each of the statistical model equations was represented through the variance covariance matrix of the estimated statistical model. This represents not only the variance of the estimated coefficients, but also their covariance, allowing the probabilistic sensitivity analysis to account for both the parameter uncertainty, and the potential correlation between input parameters. The method of Cholesky decomposition [23] was employed to correlate draws from a multivariate normal distribution of parameter values within each equation. This uncertainty was propagated through the model structure, in order to estimate the uncertainty associated with the lifetime cost and QALY predictions of the model. This estimation of uncertainty is conditional on the individual characteristics of the patient and provides a patient-characteristic specific estimate of uncertainty in the predictions of the model. Heterogeneity is represented by the differing predictions for patients with different characteristics, while uncertainty is estimated given a set of patient characteristics. Uncertainty estimates are therefore nested within the estimated heterogeneity and are handled separately by the model.

\section{RESULTS}




\section{Validating model projections at three years}

Table 1 shows the predicted results for each of the three years of the model, for overall survival, disease progression attributes, and HRQoL. Figure 5a compares the predicted and observed survival for the three years of ECLIPSE. For FEV ${ }_{1}$, the estimated annual decline based on the ECLIPSE study has previously been reported as $33 \mathrm{~mL}(95 \% \mathrm{CI}: 29,37)$ [24]. This is a relatively good fit with the estimated $27 \mathrm{~mL}$ annual decline estimated using the statistical equations that control for changes in other predictors of the disease progression attributes [13], which becomes a 39mL decline once the effects of moderate and severe exacerbations on $\mathrm{FEV}_{1}$ are taken into account. Figure 5 also shows the observed and predicted values of $\%$ predicted $\mathrm{FEV}_{1}$, moderate and severe exacerbations, symptoms, and HRQoL as measured by SGRQ Total score over the first three years of the model. The annual rate of total and severe exacerbations in the first year was previously reported as 1.21 and 0.22 , respectively (CI not reported) [22]. This is comparable with 1.20 and 0.21 total and severe exacerbations per patient as predicted by the model. The annual decline in 6MWD for ECLIPSE patients was previously reported as $5.7 \mathrm{~m}$ (CIs not reported) [25]. This is comparable with the $7.8 \mathrm{~m}$ decline estimated using the statistical equation [13], which becomes $6.1 \mathrm{~m}$ when the effects of lung function, exacerbations and symptoms over time are taken into account. The predicted SGRQ-C values were significantly different from the observed values in years 2 and 3. In general terms, the predictions of lung function and severe exacerbations were closer to the reported values for ECLIPSE than the predicted values for moderate exacerbations, symptoms, mortality, and quality of life.

\section{Heterogeneity of costs and QALYs}

Averaging over the predicted cost and QALY outcomes for the different patients within the ECLIPSE cohort gives an estimated lifetime cost of $£ 25,214$ (undiscounted) and $£ 20,318$ (discounted) and lifetime QALY of 6.45 (undiscounted) and 5.24 (discounted) per patient in the ECLIPSE cohort. As described above, results from the model predictions are individualized based on the characteristics of the patient. However, the average per-patient lifetime costs and QALYs presented above mask the differences in the individual patient predictions. Figure 2 shows the joint distribution of predicted lifetime costs and QALYs for the 1957 patients from ECLIPSE, based on the data for those patients with a full dataset available at baseline. In addition to the joint distribution on the cost-effectiveness plane, the univariate distributions for lifetime cost and QALYs are shown. 
As is commonly observed in practice, both health outcomes and costs are predicted to be positively skewed across patients.. The correlation between predicted cost and QALY outcomes was modest, with an estimated correlation coefficient of 0.08 , reflecting the competing influences that patients who live longer generally incur more costs, but that those with shorter survival are generally in more advanced stages of disease and cost more.

\section{Lifetime projections by history of exacerbation}

Figure 3 shows the projected survival (undiscounted, Figure 3a) and total accumulated cost (discounted, Figure 3b) for an average patient in ECLIPSE within two subgroups: those with no history of exacerbation at study entry, and those with a history of exacerbation at study entry. It is clear from the two panels of Figure 3 that history of exacerbation is associated with lower survival and increased cost over the lifetime of the patient.

\section{Analysis of uncertainty}

A probabilistic sensitivity analysis was employed to explore the uncertainty in lifetime costs and QALYs for two types of patient based on ECLIPSE equations: those with no history of exacerbation at study entry, and those with a history of exacerbation at study entry. These results are presented on the costeffectiveness plane in Figure 4. Summary results of the uncertainty analysis reveal uncertainty in estimates for these two patient subgroups: the additional QALYs was 0.48 for the no-exacerbation history group (95\% CI: $1.15,1.91)$ with the additional costs for the exacerbation group being $£ 4961$ (95\% CI: $-£ 491, £ 11,607)$ more expensive.

\section{DISCUSSION}


We have presented a new model of COPD that was constructed from an initial conceptualization of the disease, and validated using the data source that was used to estimate model parameters. To date, most other models have employed state transition models based on $\%$ predicted $\mathrm{FEV}_{1}$ as a measure of disease progression. In the present analysis, we have broadened the definition of COPD progression to incorporate exacerbations, lung function, exercise capacity, and symptoms as central attributes of the condition. This approach is consistent with a more contemporary definition of disease severity based on the multifactorial understanding of COPD [79]. Although all previous models have incorporated exacerbations they have tended to do so based on the consideration that this is a state that is dependent only on \% predicted FEV, and without estimating the relationship between exacerbations and disease progression. One exception is the Dutch population model of COPD, where each exacerbation causes the $\mathrm{FEV}_{1} \%$ predicted to decline with $0.19 \%$ (95\% CI: 0.092, 0.29) [26].

The implementation of the model is also novel. The use of a linked equations approach is not commonly used in HTA modeling, though it has clear links to the epidemiological literature of causal modeling. The approach was made possible through the existence of the ECLIPSE study, which provided a rich source of data from which to estimate the interconnected relationships that defined our conceptual model. Furthermore, we were able to show that the model was able to predict back the outcomes of the ECLIPSE study - although the less than perfect fit in some cases was due to the fact that the structure imposed by the conceptual model required all equations to be fit in a particular way, with particular interdependencies. For example, the conceptual model suggested that exacerbation history was expected to impact lung function [12], while lung function was expected to in turn impact the chance of exacerbation, and the empirical results confirmed this to be the case [13]. The resulting model estimated both jointly, adding structure to the analysis. An unrestricted prediction model of each outcome independently without representing those interdependencies would undoubtedly have provided a better fit to the data, but would have lost the richness of the interdependencies the current model is able to estimate. It is these interdependencies that allow the estimation of the impact of treatment that is shown to affect a surrogate endpoint (e.g., symptoms and/or FEV $1 \%$ predicted) on final outcomes.

ECLIPSE was an observational study, designed to gain a better understanding of COPD as a disease. This made it ideal in many respects for developing a new model of COPD disease progression. The purpose of developing the present model was to provide a method for estimating long-term treatment effects where new and existing treatments for COPD are observed to impact on a variety of prognostic markers. We anticipate that 
the model is most likely to be used when treatment is shown to impact a combination of the disease progression attributes of exacerbations, lung function, exercise capacity, or symptoms.

One of the features of the Galaxy COPD model is the way in which it estimates patient-characteristic specific uncertainty in a manner that captures uncertainty in prognosis for individual patients with different characteristics. In this way, the model is able to represent uncertainty in the predicted costs and QALY outcomes based on the estimated parameters, which include parameters describing heterogeneity between individuals. The uncertainty analysis is therefore nested within the analysis of heterogeneity with the consequence that while uncertainty can be quantified for individual patient predictions (or equivalently for homogeneous cohorts) it is not clear how uncertainty can be represented in the mean outcomes of heterogeneous cohorts.

The strengths of our approach include explicit documenting of an underlying conceptual model, and validation of the model constructed according to best practice guidelines $[10,11,27]$. Furthermore, the model incorporates not only the effect of lung function, but also exacerbations, exercise capacity, and symptoms. When combined with baseline prognostic variables, this results in a highly flexible model that can generate predicted changes in QALYs and health service resource costs associated with a wide variety of potential treatments or disease management strategies for COPD, as well as explore potential subgroup analyses. Treatment effects can be incorporated into the model in two ways. Firstly, if there is evidence of the treatment effect on a given surrogate endpoint included in the model, the effect of changes to this endpoint on the final cost, survival, and HRQoL (QALY) outcomes can be estimated. Secondly, if direct evidence of the treatment effect on both surrogate and final outcomes is available, then the model can estimate the central association corrected treatment effect that acts independently of the central associations.

Nevertheless, there are limitations to the modeling approach adopted. Firstly, the model is based on a single, albeit large, cohort study of COPD, and therefore the generalizability of the model is dependent on the generalizability of the ECLIPSE study cohort. The linked equations approach to modeling is likely to be unfamiliar to many potential users of the model and this may limit its acceptance by decision makers. For this reason, an extensive validation of the model predictions is required. An internal validation against the ECLIPSE data was presented, and revealed that in seeking to predict a number of different outcomes simultaneously, the fit achieved was not as good as if a single model had been used to fit each of these outcomes independently. Nevertheless, the ability of a model to fit the data used to estimate its parameters is a necessary, but not 
sufficient, condition for validation. In practice, external validation (i.e., the ability of the model to predict data not used in its construction) is much more important than internal validation. In the next paper of the series, we will report the ability of the model to predict outcomes in two large clinical trials that included COPD patients: the TORCH study [15] and the Understanding Potential Long-term Impacts on Function with Tiotropium (UPLIFT) study [28].

\section{CONCLUSIONS}

We have reported the conceptualization, implementation, and internal validation of a new form of model for COPD that is based on a series of linked equations estimated from a longitudinal cohort study of COPD patients. This Galaxy COPD model is capable of predicting COPD outcomes for a variety of potential treatment effects on intermediate risk factors in both the short and long term. Further external validation of short- and long-term predictions of the model is required.

\section{ACKNOWLEDGMENTS}

Editorial support in the form of editorial suggestions to draft versions of this paper, , copyediting, referencing and graphic services was provided by Laura Maguire, MChem at Gardiner-Caldwell Communications (Macclesfield, UK) and was funded by GSK.

\section{AUTHOR CONTRIBUTIONS}

AB, MC, NL, DL, MT, and HM were involved in the conception and design of the study, data analysis, and data interpretation; TB, NR, SG, AE, and CC contributed to the conception and design of the 
study/model and performed programming, data analysis, and reporting; MRvM was involved in developing the conceptual model, designing the study, and interpreting the data. She reviewed the article critically and approved the final version that was submitted; ASI contributed to the design, analysis, and data interpretation.

\section{AUTHOR CONFLICTS OF INTEREST}

MC, AI, MT, HM, NL are employees of and hold stock in GSK. SGM was an employee of GSK at the time of the research and analyses of this project, and is currently an employee of Amgen.

AB, TB, NR, AE, CC have received funding as a consultant and through Oxford Outcomes Ltd (now known as ICON Plc) from GSK related to the development of this model.

MRvM has published on other economic models of chronic obstructive pulmonary disease, either developed with public funding or pharmaceutical industry funding. She has received a fee for participating in the Steering Group. In recent years she has received unrestricted research grants from Astellas (Nycomed/Takeda), Boehringer Ingelheim, and GSK. She also received speaker's fees from these companies.

DAL has received grant support, honoraria and consultancy fees from GSK. He is the Chair of the GSK Respiratory Therapy Area Board. 


\section{REFERENCES}

1. National Clinical Guideline Centre. Chronic obstructive pulmonary disease. http://www.ncgc.ac.uk/Guidelines/Published/20. Accessed 4 March 2015.

2. Global Initiative for Chronic Obstructive Lung Disease (GOLD). Global Strategy for the Diagnosis, Management and Prevention of COPD. http://www.goldcopd.org/guidelines-global-strategy-for-diagnosismanagement.html. Accessed 4 March 2015.

3. NHS Information Centre for Health and Social Care. NHS Information Centre for Health and Social Care, London. http://www.hscic.gov.uk/. Accessed 4 March 2015.

4. British Thoracic Society. The Burden of Lung Disease. 2nd ed. London: British Thoracic Society; 2006.

5. Starkie HJ, Briggs AH, Chambers MG. Pharmacoeconomics in COPD: lessons for the future. Int J Chron Obstruct Pulmon Dis. 2008;3(1):71-88.

6. Hoogendoorn M, Feenstra TL, Asukai Y, Borg S, Hansen RN, Jansson S, et al. Cost-effectiveness models for chronic obstructive pulmonary disease: cross-model comparison of hypothetical treatment scenarios. Value Health. 2014;17(5):525-36.

7. Agustí AG, Noguera A, Sauleda J, Sala E, Pons J, Busquets X. Systemic effects of chronic obstructive pulmonary disease. Eur Respir J. 2003;21(2):347-60.

8. Celli BR, Cote CG, Marin JM, Casanova C, Montes de Oca M, Mendez RA, et al. The body-mass index, airflow obstruction, dyspnea, and exercise capacity index in chronic obstructive pulmonary disease. $\mathrm{N}$ Engl J Med. 2004;350(10):1005-12.

9. Briggs A, Spencer M, Wang H, Mannino D, Sin DD. Development and validation of a prognostic index for health outcomes in chronic obstructive pulmonary disease. Arch Intern Med. 2008;168(1):71-9.

10. Caro JJ, Briggs AH, Siebert U, Kuntz KM; ISPOR-SMDM Modeling Good Research Practices Task Force. Modeling good research practices overview: a report of the ISPOR-SMDM Modeling Good Research Practices Task Force-1. Med Decis Making. 2012;32(5):667-77.

11. Roberts M, Russell LB, Paltiel AD, Chambers M, McEwan P, Krahn M; ISPOR-SMDM Modeling Good Research Practices Task Force. Conceptualizing a model: a report of the ISPOR-SMDM Modeling Good Research Practices Task Force-2. Med Decis Making. 2012;32(5):678-89.

12. Tabberer M, Gonzalez McGuire S, Muellerova H, Briggs AH, Rutten van-Molken M, Chambers M, et al. Development of a conceptual model for use in disease progression and economic modeling of chronic 
obstructive pulmonary disease. Medicial Decision Making. 2015 (companion paper submitted 20 February 2015; manuscript ID: MDM-15-059).

13. Exuzides A, Colby C, Briggs A, Lomas DA, Rutten van-Mölken M, Tabberer M, et al. Statistical modeling of disease progression for chronic obstructive pulmonary disease using data from the ECLIPSE study. Medicial Decision Making. 2015 (Epub ahead of print).

14. Agusti A, Calverley PM, Celli B, Coxson HO, Edwards LD, Lomas DA, et al.; Evaluation of COPD Longitudinally to Identify Predictive Surrogate Endpoints (ECLIPSE) investigators. Characterisation of COPD heterogeneity in the ECLIPSE cohort. Respir Res. 2010;11:122.

15. Calverley PM, Anderson JA, Celli B, Ferguson GT, Jenkins C, Jones PW, et al.; TORCH investigators. Salmeterol and fluticasone propionate and survival in chronic obstructive pulmonary disease. N Engl J Med. 2007;356(8):775-89.

16. Jones PW, Forde Y. St George's Respiratory Questionnaire for COPD Patients (SGRQ-C): Manual. London: University of London; 2012.

17. British National Formulary (BNF). http://www.bnf.org/bnf/index.htm. Accessed 4 March 2015.

18. Personal Social Services Research Unit (PSSRU). Unit Costs of Health \& Social Care 2011. http://www.pssru.ac.uk/pdf/uc/uc2011/uc2011.pdf . Accessed 4 March 2015.

19. Department of Health. 2010-11 reference costs. http://www.dh.gov.uk/en/Publicationsandstatistics/Publications/PublicationsPolicyAndGuidance/DH_1311 40. Accessed 4 March 2015.

20. National Institute of Health and Care Excellence (NICE). Guide to the methods of technology appraisal. http://www.nice.org.uk/article/PMG9/chapter/Foreword. Accessed 4 March 2015.

21. Starkie HJ, Briggs AH, Chambers MG, Jones P. Predicting EQ-5D values using the SGRQ. Value Health. $2011 ; 14(2): 354-60$.

22. Hurst JR, Vestbo J, Anzueto A, Locantore N, Müllerova H, Tal-Singer R, et al.; Evaluation of COPD Longitudinally to Identify Predictive Surrogate Endpoints (ECLIPSE) investigators. Susceptibility to exacerbation in chronic obstructive pulmonary disease. N Engl J Med. 2010;363(12):1128-38.

23. Briggs AH, Claxton K, Sculpher MJ. Decision Modelling for Health Economic Evaluation. Oxford: Oxford University Press; 2006.

24. Vestbo J, Edwards LD, Scanlon PD, Yates JC, Agusti A, Bakke P, et al.; ECLIPSE Investigators. Changes in forced expiratory volume in 1 second over time in COPD. N Engl J Med. 2011;365(13):1184-92. 
25. Spruit MA, Polkey MI, Celli B, Edwards LD, Watkins ML, Pinto-Plata V, et al.; Evaluation of COPD Longitudinally to Identify Predictive Surrogate Endpoints (ECLIPSE) study investigators. Predicting outcomes from 6-minute walk distance in chronic obstructive pulmonary disease. J Am Med Dir Assoc. 2012;13(3):291-7.

26. Hoogendoorn M, Rutten-van Mölken MP, Hoogenveen RT, Al MJ, Feenstra TL. Developing and applying a stochastic dynamic population model for chronic obstructive pulmonary disease. Value Health. 2011;14(8):1039-47.

27. Eddy DM, Hollingworth W, Caro JJ, Tsevat J, McDonald KM, Wong JB; ISPOR-SMDM Modeling Good Research Practices Task Force. Model transparency and validation: a report of the ISPOR-SMDM Modeling Good Research Practices Task Force-7. Med Decis Making. 2012;32(5):733-43.

28. Tashkin DP, Celli B, Senn S, Burkhart D, Kesten S, Menjoge S, et al.; UPLIFT Study Investigators. A 4year trial of tiotropium in chronic obstructive pulmonary disease. N Engl J Med. 2008;359(15):1543-54. 
Table 1 Model Predictions Over the 3-Year Timeframe of ECLIPSE

\begin{tabular}{|c|c|c|c|c|c|c|c|c|}
\hline \multirow[b]{2}{*}{$\begin{array}{l}\text { Individual simulations } \\
n=1957\end{array}$} & \multicolumn{2}{|c|}{ Baseline } & \multicolumn{2}{|c|}{ Year 1} & \multicolumn{2}{|c|}{ Year 2} & \multicolumn{2}{|c|}{ Year 3} \\
\hline & $\begin{array}{c}\text { Mean } \\
(95 \% \mathrm{CI})\end{array}$ & SD & $\begin{array}{c}\text { Mean } \\
(95 \% \mathrm{CI})\end{array}$ & SD & $\begin{array}{c}\text { Mean } \\
(95 \% \mathrm{CI})\end{array}$ & SD & $\begin{array}{c}\text { Mean } \\
(95 \% \mathrm{CI})\end{array}$ & SD \\
\hline Predicted survival, \% & 100.0 & & $\begin{array}{c}96.9 \\
(96.1,97.6)\end{array}$ & 2.8 & $\begin{array}{c}92.2 \\
(91,93.4)\end{array}$ & 6.5 & $\begin{array}{c}86.9 \\
(85.4,88.4)\end{array}$ & 10.3 \\
\hline FEV $_{1} \%$ predicted, \% & $\begin{array}{c}48.1 \\
(47.4,48.8)\end{array}$ & 15.6 & $\begin{array}{c}47.0 \\
(46.3,47.7)\end{array}$ & 15.5 & $\begin{array}{c}46.1 \\
(45.4,46.8)\end{array}$ & 15.3 & $\begin{array}{c}45.2 \\
(44.5,45.8)\end{array}$ & 15.2 \\
\hline $\mathrm{FEV}_{1}, \mathbf{m L}$ & $\begin{array}{c}1343 \\
(1320,1365)\end{array}$ & 513 & $\begin{array}{c}1302 \\
(1280,1324)\end{array}$ & 507 & $\begin{array}{c}1265 \\
(1243,1287)\end{array}$ & 499 & $\begin{array}{c}1227 \\
(1206,1249)\end{array}$ & 492 \\
\hline $\begin{array}{l}\text { Total exacerbations in } \\
\text { previous } 12 \text { months }\end{array}$ & & & $\begin{array}{c}1.236 \\
(1.204,1.267)\end{array}$ & 0.711 & $\begin{array}{c}1.215 \\
(1.184,1.247)\end{array}$ & 0.713 & $\begin{array}{c}1.205 \\
(1.173,1.237)\end{array}$ & 0.720 \\
\hline Moderate, count & & & $\begin{array}{c}1.032 \\
(1.007,1.057)\end{array}$ & 0.570 & $\begin{array}{c}0.968 \\
(0.945,0.992)\end{array}$ & 0.537 & $\begin{array}{c}0.908 \\
(0.885,0.930)\end{array}$ & 0.503 \\
\hline Severe, count & & & $\begin{array}{c}0.204 \\
(0.196,0.212)\end{array}$ & 0.182 & $\begin{array}{c}0.247 \\
(0.237,0.257)\end{array}$ & 0.222 & $\begin{array}{c}0.297 \\
(0.286,0.309)\end{array}$ & 0.269 \\
\hline
\end{tabular}




\section{Dyspnea symptoms}

Most days, \%

Several days, \%

None, \%

\section{Cough and sputum}

With criteria, *\%

48.3

$(46.1,50.5)$

Without criteria, ${ }^{\dagger} \%$

\section{SGRQ-C score}

$(55.9,60.3)$

$$
\begin{gathered}
35.3 \\
(33.2,37.4)
\end{gathered}
$$

$$
51.7
$$

50
(49.1, 50.9)

6MWD, m

$$
\begin{gathered}
369.9 \\
(364.5,375.3)
\end{gathered}
$$

29.4

58.8

$(56.6,60.9)$

22.3

34.7

$(32.6,36.8)$

6.5

$$
47.5
$$

$(45.3,49.7)$

52.5

20.164

51.2

$(50.3,52.2)$

121.346

362.6

$(357.3,368.0)$
29.4

60.1

$(57.9,62.2)$

33.5

$(31.4,35.6)$

6.4

46.9

$(44.6,49.1)$

53.1

52.2

$(51.3,53.2)$

121.393
29.3

61.3

$(59.2,63.5)$

32.4

$(30.4,34.5)$

6.3

46.2

$(44,48.4)$

53.8

53.2

20.755

$(52.3,54.2)$

$$
343.4
$$

117.986

6MWD, six-minute walk distance; $\mathrm{FEV}_{1}$, forced expiratory volume in one second; SD, standard deviation; SGRQ-C, St. George's Respiratory Questionnaire for COPD.

*With criteria: cough and/or sputum most days per week; ${ }^{\dagger}$ Without criteria: cough and/or sputum not at all or several days per week 
Figure 1. Linking baseline prognostic variables, through disease progression attributes, to final COPD outcomes. Panel (a) shows the relationship between the central attributes in the different time periods. Panel (b) shows the relationship between the central attributes and the final health outcomes.

a)

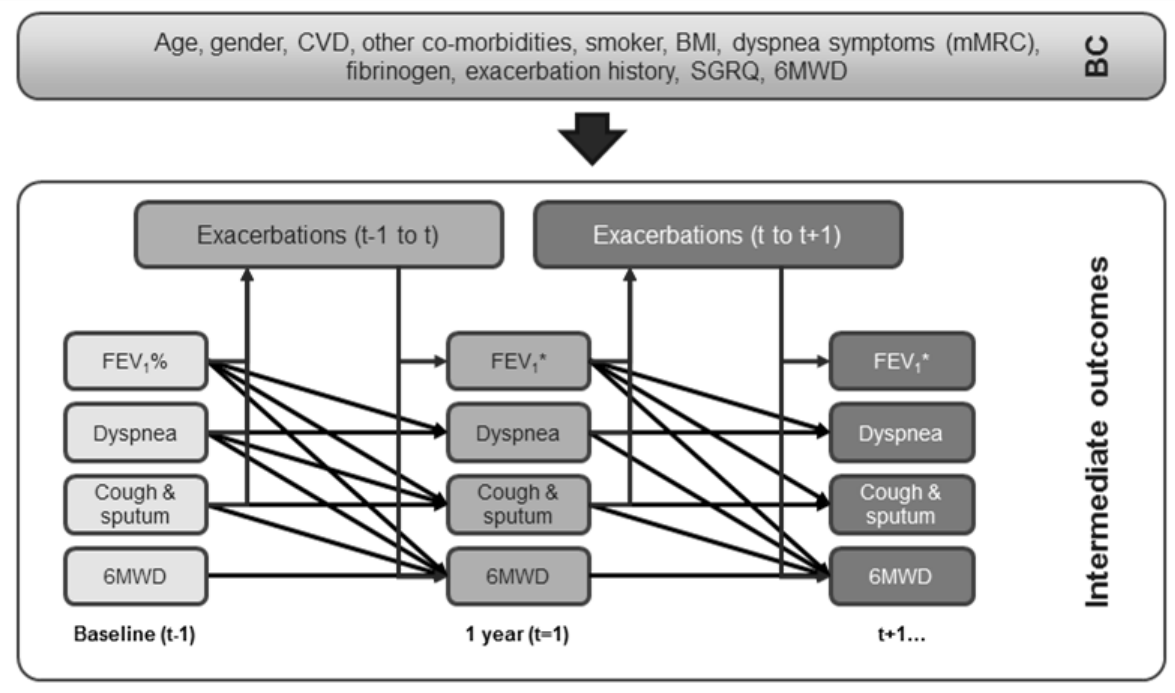

b) Age, gender, CVD, other co-morbidities, smoker, BMl, dyspnea symptoms (mMRC),
fibrinogen, exacerbation history, SGRQ, 6MWD
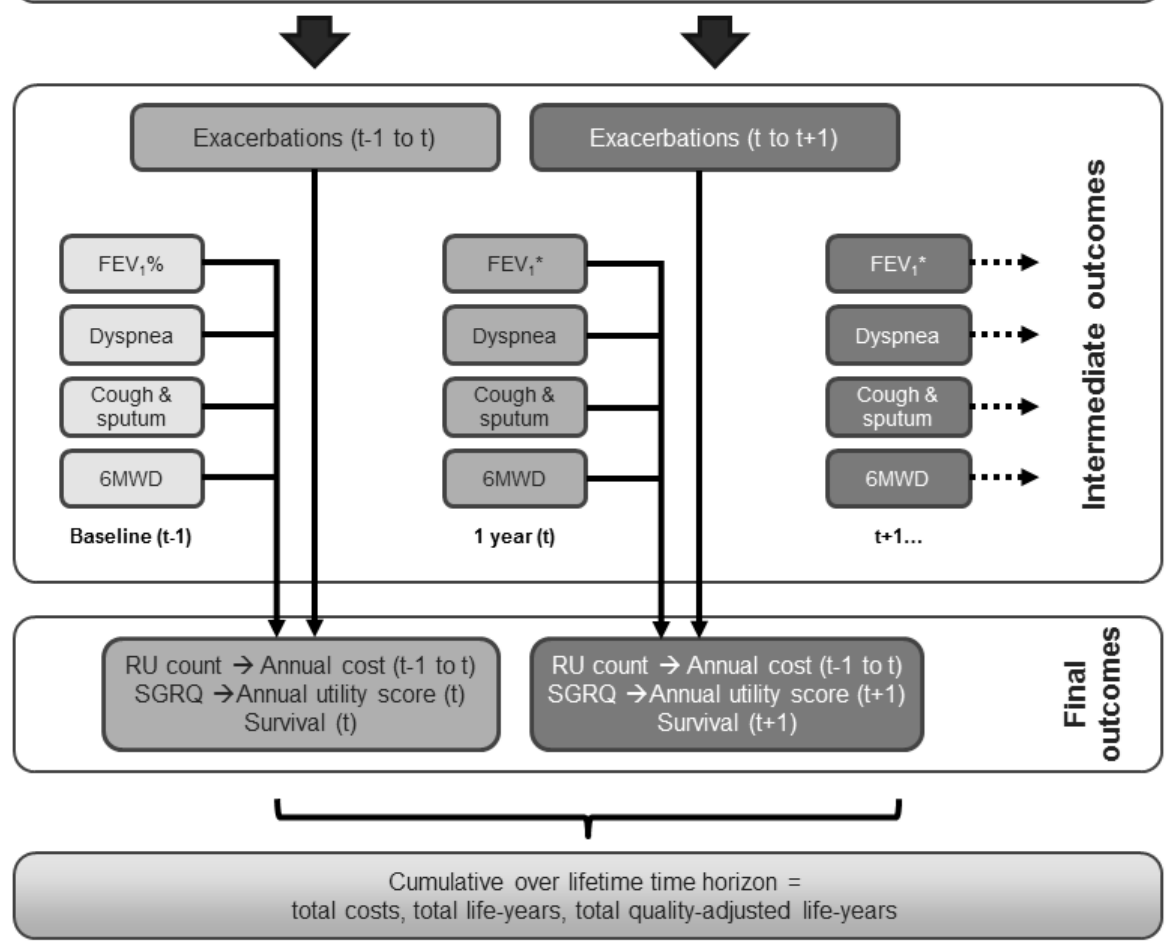
The associations between disease progression attributes shown in Figure 1a are omitted to aid clarity of presentation. COPD, chronic obstructive pulmonary disease; CVD, cardiovascular disease; BMI, body mass index; mMRC, modified Medical Research Council questionnaire; SGRQ, St George's Respiratory Questionnaire score; $6 M W D$, six-minute walking distance; $B C$, baseline covariates; $F E V_{1}$, forced expiratory volume in one second; $R U$, resource utilization; $t$, time; $Q A L Y$, quality-adjusted life years. *FEV $(m L)$ was calculated using the risk equation at year ' $t$ ' and converted to $F E V_{1} \%$ predicted based on the cohort profile. 
Figure 2. Joint distribution of predicted lifetime costs and QALYs for individual patients on the cost-

effectiveness plane together with histograms of the cost and QALY histograms showing heterogeneity across the 1957 patients with complete data in ECLIPSE.

Lifetime costs (in $£ 000$ s) on natural log scale. QALY, quality-adjusted life years.
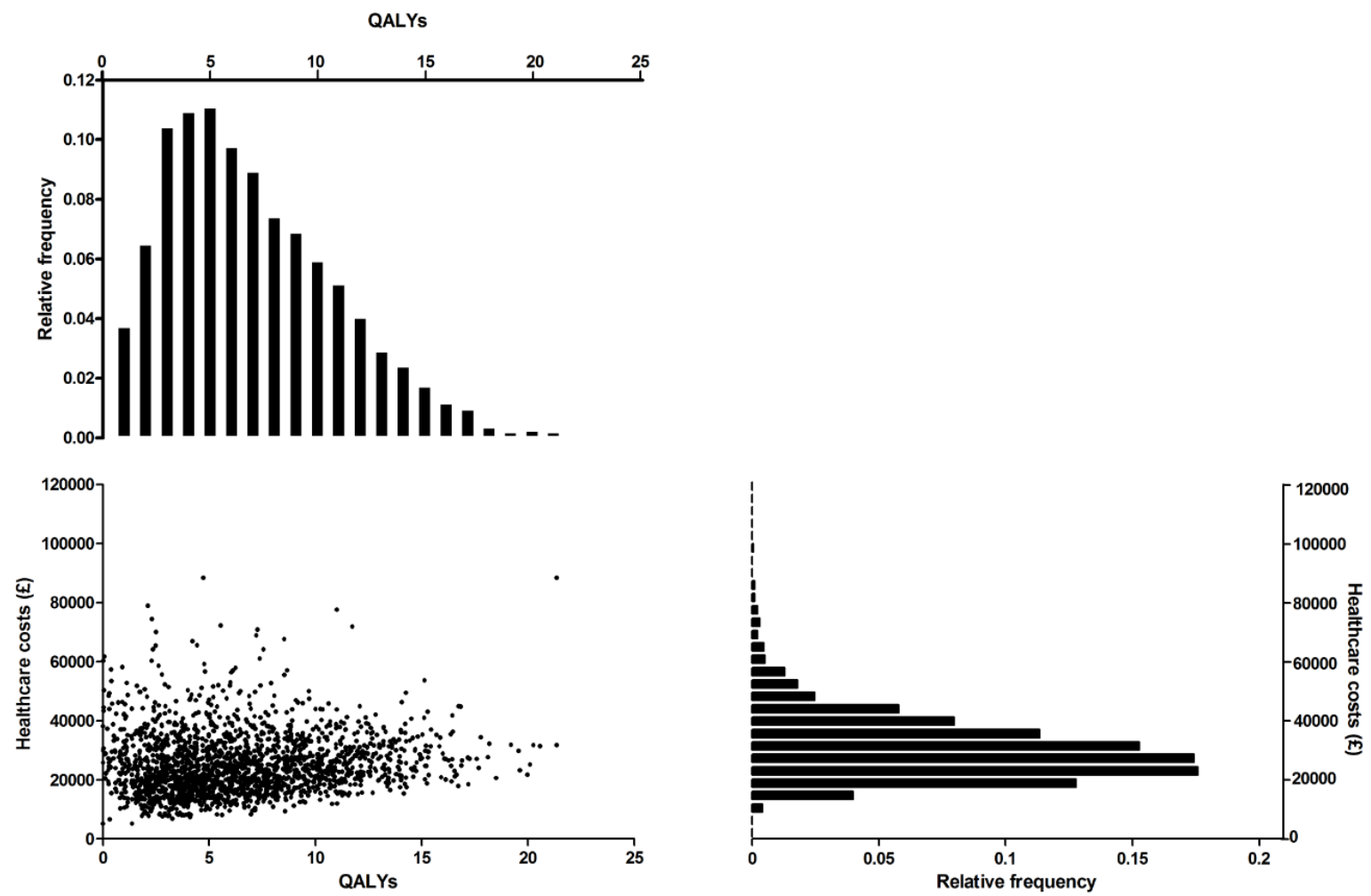
Figure 3. Lifetime projections for ECLIPSE cohort of (a) survival and $(b)$ cumulative costs for patients with and without prior exacerbations.

(a)

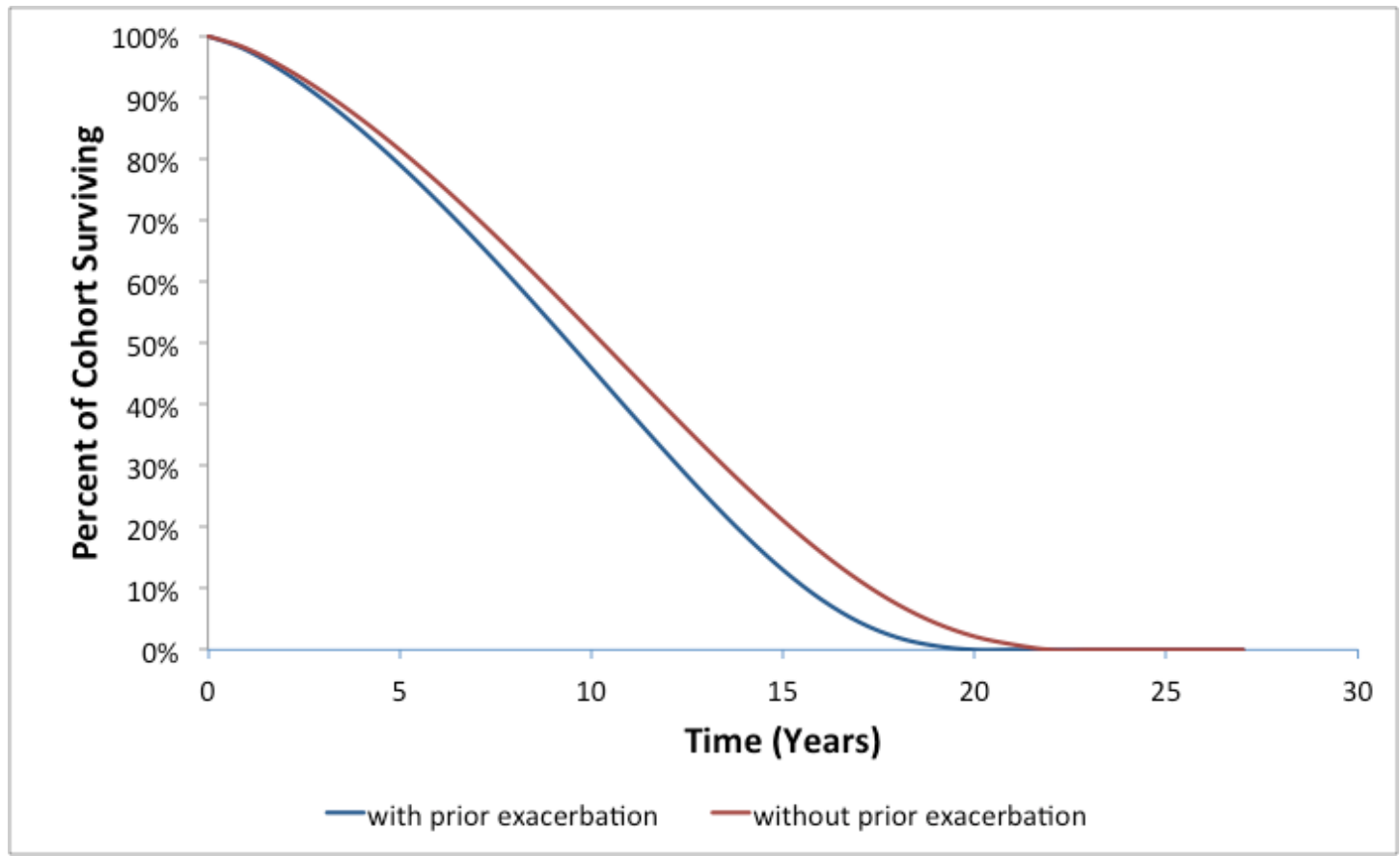

(b)

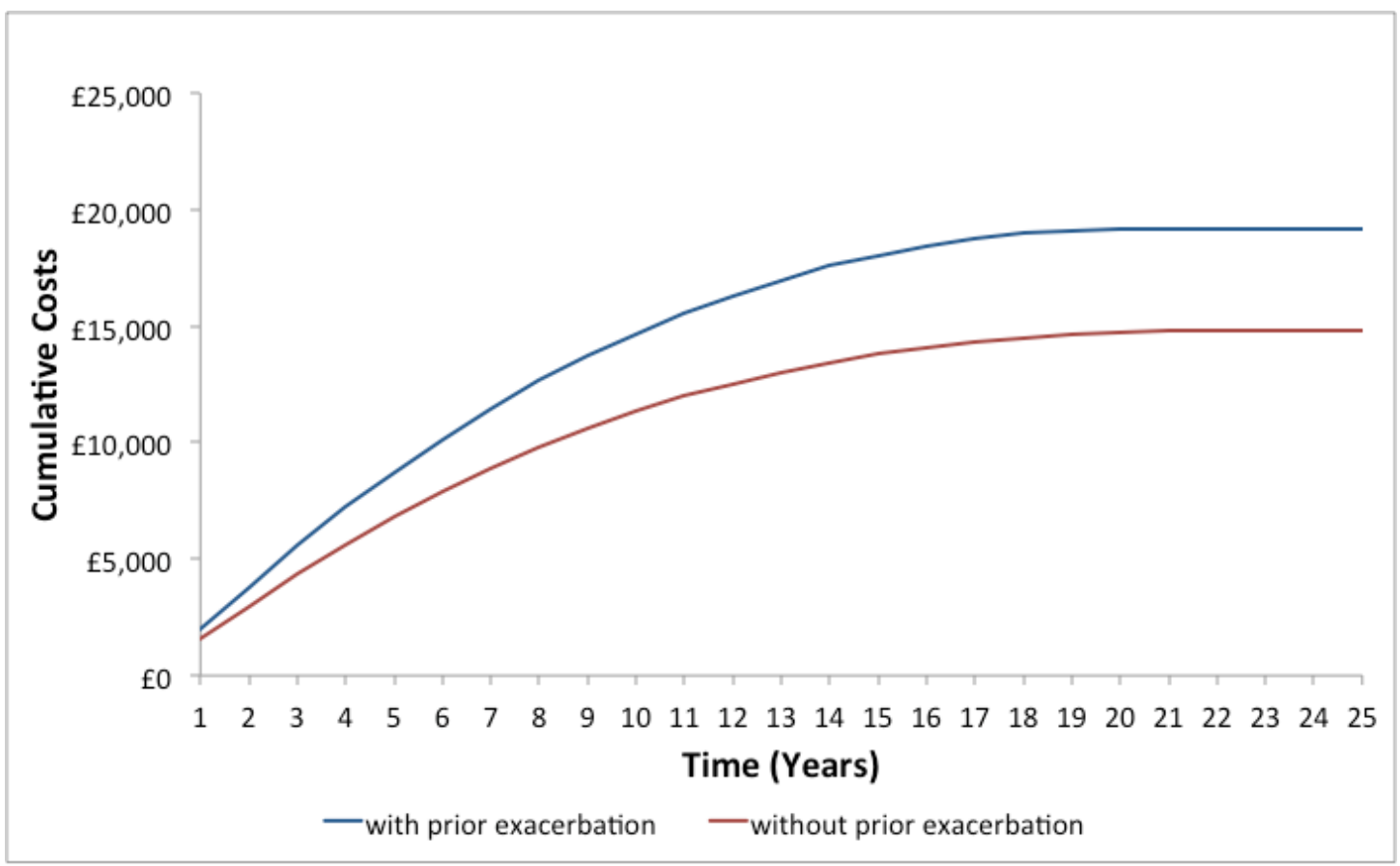


Figure 4. Illustration of probabilistic uncertainty for ECLIPSE subgroups on the cost-effectiveness plane for (a) patients with no history of exacerbation and $(b)$ patients with a history of exacerbations.

(a)

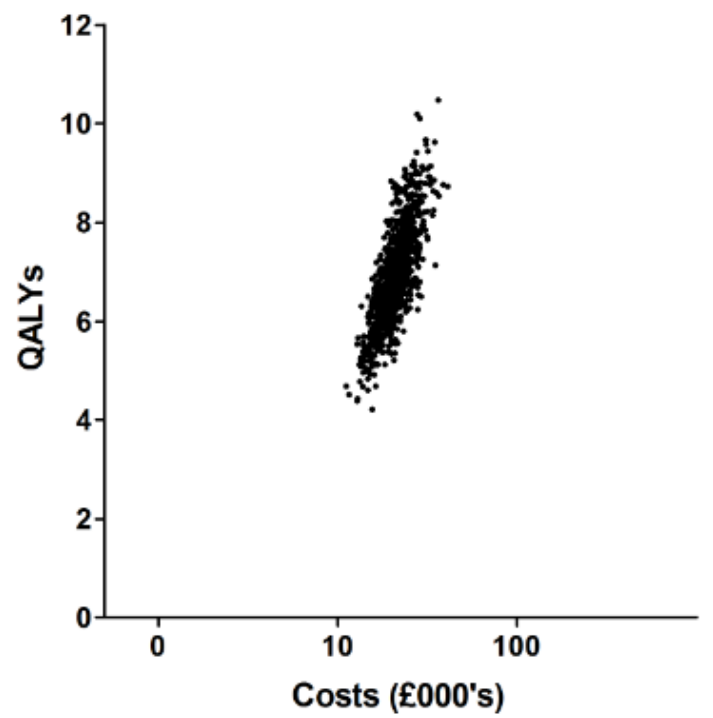

(b)

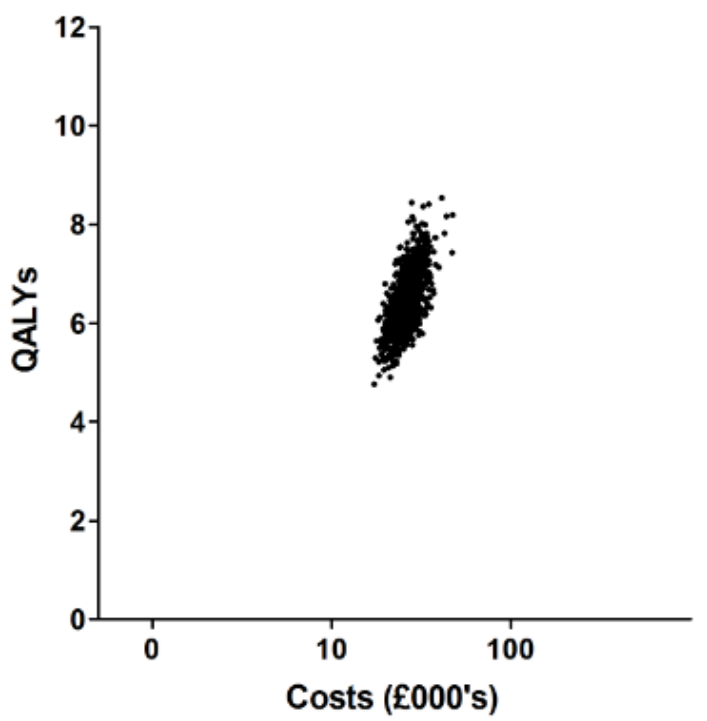

QALY, quality-adjusted life years. 
Figure 5. Observed versus predicted outcomes from ECLIPSE over three years for (a) survival (b) \% predicted $F E V_{l}(c)$ moderate and severe exacerbations $(d)$ dyspnea symptoms $(e)$ cough and/or sputum symptoms and $(f)$ SGRQ-C score.

a)

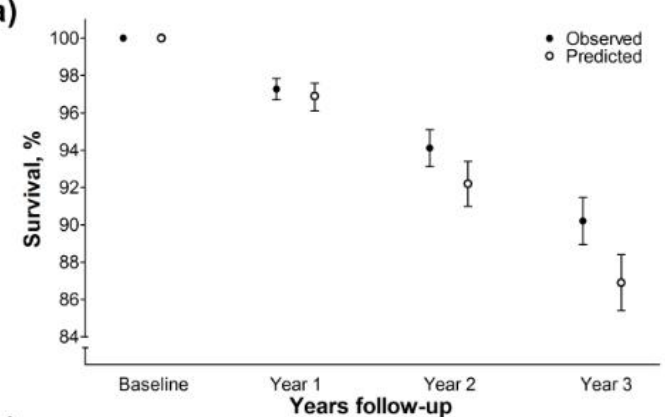

c)
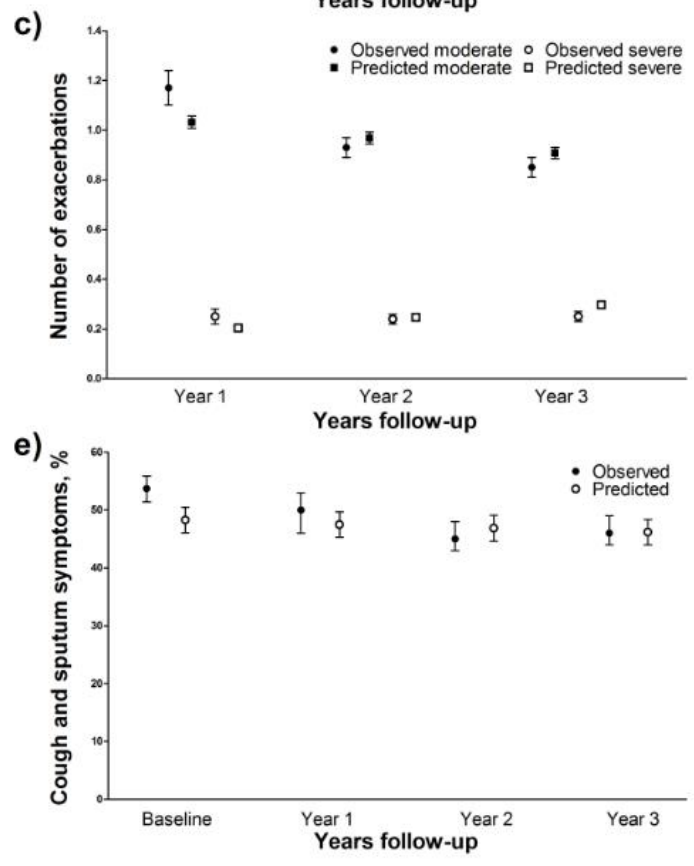

b)

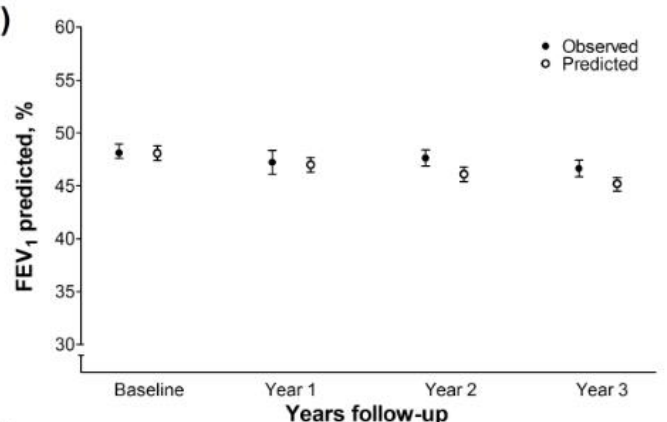

d)

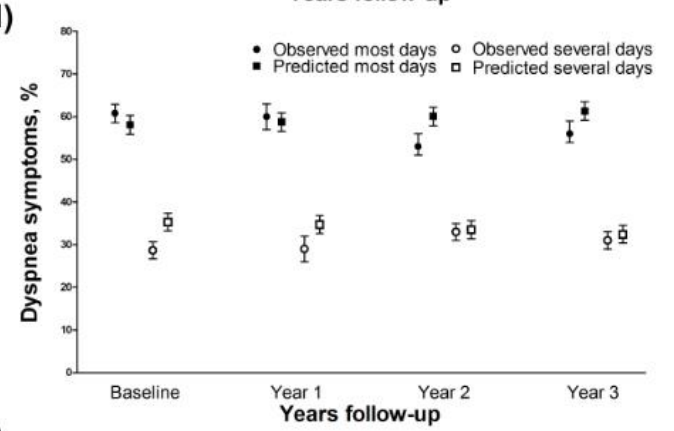

f)

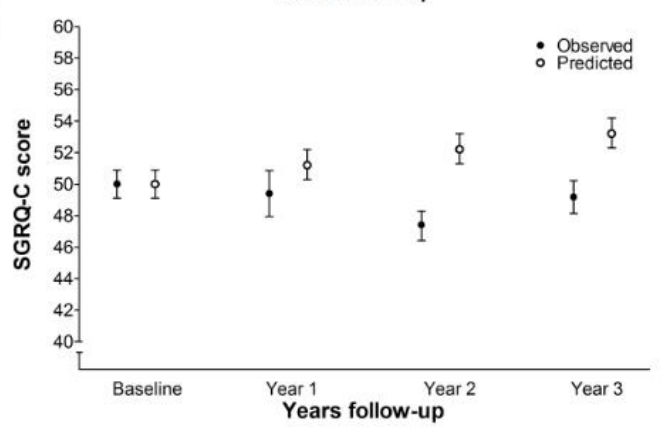

$F E V_{1}$, forced expiratory volume in one second; SGRQ-C, St George's Respiratry Questionnaire for COPD. 
Development of the Galaxy Chronic Obstructive Pulmonary Disease

(COPD) Model Using Data from ECLIPSE: Internal Validation of a

\section{Linked Equations Cohort Model}

Andrew Briggs, DPhil*, Timothy Baker, BSc, Nancy A. Risebrough, BA, Mike Chambers, MSc, Sebastian Gonzalez-McQuire, MSc', Afisi S. Ismaila, PhD, Alex Exuzides, PhD, Chris Colby, PhD, Maggie Tabberer, MSc, Hana Muellerova, PhD, Nicholas Locantore, PhD, Maureen Rutten vanMölken, PhD, David A. Lomas, PhD FRCP

\section{Online supplementary material}

The TORCH study included three years'follow-up for every patient without censoring. The equations reported represent the estimated yearly resource use estimated from the three years of resource use data collected in TORCH 
Table A1 Baseline Patient Characteristics for ECLIPSE and TORCH

ECLIPSE

$(n=1957)$

$(n=6108)$

\section{Baseline characteristics}

Mean age (SD), yrs

$63.4(7.1)$

$65.0(8.3)$

Gender (male), \%

65.5

76.0

Mean height (SD), cm

$169.4(8.8)$

$168.7(8.8)$

Body mass index, \%

Low $\leq 20 \mathrm{~kg} / \mathrm{m}^{2}$ 
CVD comorbidity, \%

'Other' comorbidity, * \%

$\geq 1$ exacerbation in prior year, $\%$

$0.78(0.88)$

$460.5(104.8)$

Mean fibrinogen (SD), $\mathrm{mcg} / \mathrm{mL}$

mMRC score, \%

0
47.1

32.9

73.3

22.0

79.0

57.0

$0.89(0.86)$

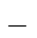

34.0

53.2
7.0

42.5

2.7

50.5 
FEV 1, mL

Mean (SD)

$95 \% \mathrm{CI}$

\section{$\mathrm{FEV}_{1} \%$ predicted}

Mean (SD)

$48.1(15.5)$

$47.4,48.8$

$95 \% \mathrm{CI}$

Dyspnea symptoms (most days per week), \%

$95 \%$ CI

Dyspnea symptoms (several days per week), \%
$1226(444)$

1215,1237

$44.0(12.4)$

$43.7,44.3$

48.0

$46.0,49.0$
45.0 
Dyspnea symptoms (none), \%

Mean

$95 \% \mathrm{CI}$

Cough or sputum (most days per week), \%

Mean

$95 \% \mathrm{CI}$

6MWD, m

Mean (SD)

$95 \% \mathrm{CI}$

\section{SGRQ-C score}

Mean (SD)
10.6

$9.22,11.9$

$7.0,8.0$
49.0

$51.4,55.9$

$369(121)$

365,375
$48.0,50.0$

$48.1(15.5)$

$49.3(17.1)$ 
SD, standard deviation; 6MWD, six-minute walking distance; CI, confidence interval; $\mathrm{CVD}$, cardiovascular disease; FEV 1 , forced expiratory volume in one second; mMRC, modified Medical Research Council questionnaire; SD, standard deviation; SGRQ-C, St George’s Respiratory Questionnaire for COPD. *`Other’ comorbidity includes any reported non-CVD comorbidity. 
APPENDIX A TORCH Resource Utilization Risk Equations

Table A2 Resource Utilization - Ward Days (excluding ICU wards)

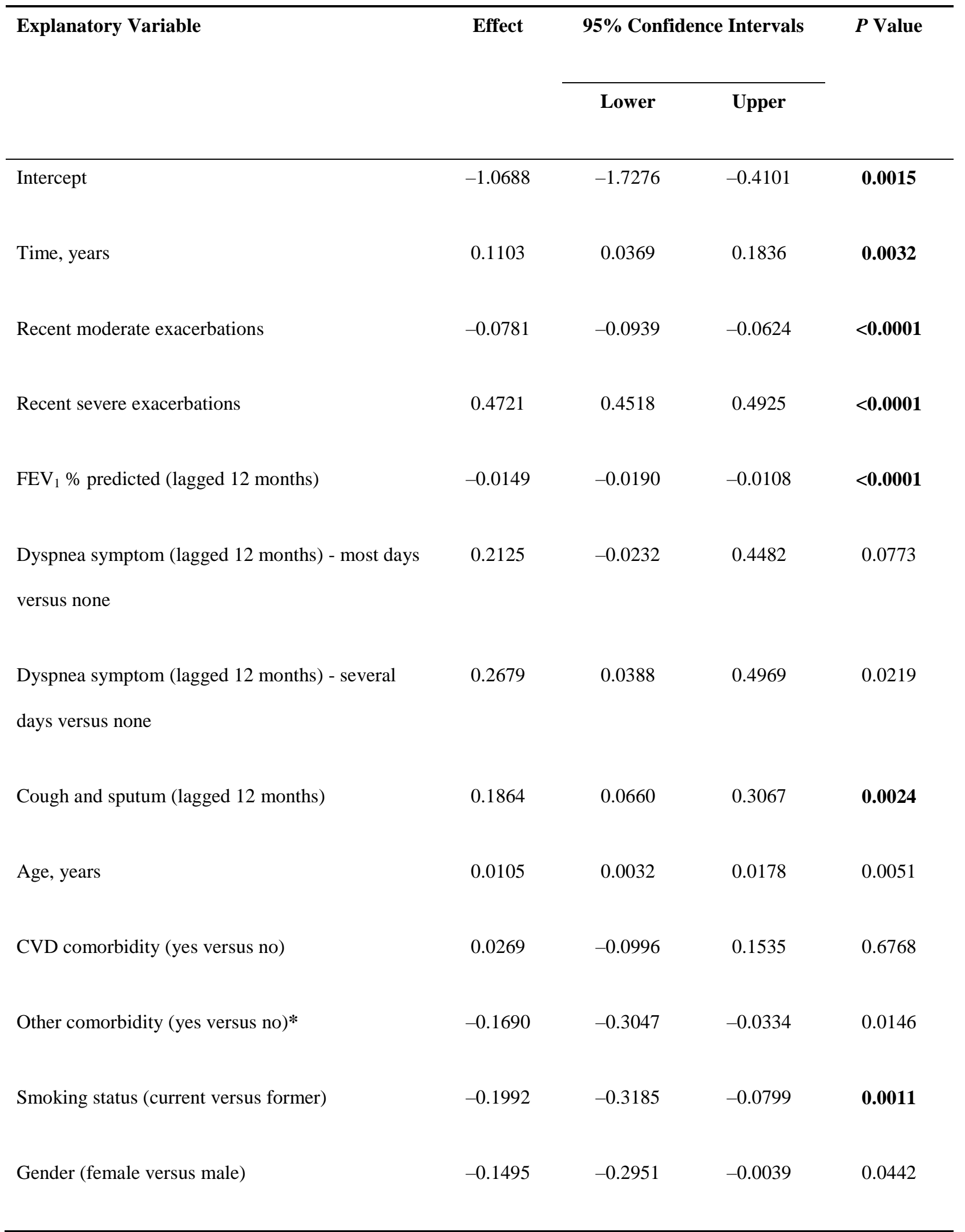




\begin{tabular}{lcccr}
\hline BMI (high versus normal) & -0.3781 & -0.5557 & -0.2005 & $<\mathbf{0 . 0 0 0 1}$ \\
BMI (low versus normal) & 0.2960 & 0.1678 & 0.4241 & $<\mathbf{0 . 0 0 0 1}$ \\
Baseline mMRC (mMRC $\geq 2)$ & & & & \\
Baseline SGRQ & -0.0046 & -0.2553 & 0.2461 & 0.9715 \\
& & & & \\
Prior exacerbations $(\geq 1$ versus 0) & 0.0147 & 0.0109 & 0.0185 & $<\mathbf{0 . 0 0 0 1}$ \\
& 0.4613 & 0.3383 & 0.5843 & $<\mathbf{0 . 0 0 0 1}$
\end{tabular}

Number of patients $=4669$

Number of observations $=11,463$

$\mathrm{AIC}=63,631.99$

AIC, Akaike information criterion; BMI, body-mass index; $\mathrm{CVD}$, cardiovascular disease; $\mathrm{FEV}_{1}$, forced expiratory volume in one second; ICU, intensive care unit; mMRC, modified Medical Research Council score;

SGRQ, St George's respiratory questionnaire. *Other, any reported non-CVD comorbidity. Bold $=P<0.05$. 
Table A3 Resource Utilization - ICU Days

Explanatory Variable
Effect

95\% Confidence Intervals

Lower Upper

$-2.1133$

0.5465

0.0245

0.4223

$-0.0238$

$-0.5286$

$-1.2178$

0.1607

0.1328

days versus none

Dyspnea symptoms (lagged 12 months) - several days versus none

Cough and sputum (lagged 12 months)

Age, years

CVD comorbidity (yes versus no)

Other comorbidity (yes versus no)*

Smoking status (current versus former)

Gender (female versus male)

BMI (high versus normal)

$-0.0624 \quad-0.6960$

0.5712

0.8470

$-0.0344$

$-0.4437$

0.3750

0.8693

$-0.0029$

$-0.0275$

0.0216

0.8153

0.6546

0.2688

1.0404

0.0009

0.3295

$-0.1954$

0.8544

0.2186

0.3026

$-0.0922$

0.6974

0.1330

$-0.9816$

$-1.5658$

$-0.3974$

0.0010

0.0403

$-0.4761$

0.5567

0.8784 
BMI (low versus normal)

Baseline mMRC $(m M R C \geq 2)$

Baseline SGRQ

Prior exacerbations ( $\geq 1$ versus 0 )

Number of patients $=4669$

Number of observations $=11,463$

$\mathrm{AIC}=10,959.29$
0.2310

$-0.7611$

$-1.3680$

$-0.1543$

0.0140

0.0142

0.0015

0.0268

0.0282

$-0.0076$

$-0.3889$

0.3737

0.9688

0.3154 
Table A4 Resource Utilization - Emergency Room Visits

Explanatory Variable
Effect

95\% Confidence Intervals

$P$ Value

\section{Lower Upper}

Intercept

Time, years

$-0.087$

$-0.2969$

0.1229

0.4168

Recent moderate exacerbations

0.076

0.0427

0.1093

$<0.0001$

Recent severe exacerbations

0.2387

0.1736

0.3038

$<0.0001$

$\mathrm{FEV}_{1} \%$ predicted (lagged 12 months)

Dyspnea symptoms (lagged 12 months) - most

0.1244

$-0.5732$

0.8219

0.7268

days versus none

Dyspnea symptoms (lagged 12 months) - several

0.1653

$-0.4980$

0.8286

0.6252

days versus none

Cough and sputum (lagged 12 months)

Age, years

CVD comorbidity (yes versus no)

Other comorbidity (yes versus no)*

Smoking status (current versus former)

Gender (female versus male)

BMI (high versus normal)
$-0.0627$

$-0.4055$

0.2801

0.7200

0.0157

$-0.0056$

0.0370

0.1489

$-0.1036$

$-0.4877$

0.2806

0.5973

$-0.2442$

$-0.6403$

0.1520

0.2270

$-0.1766$

$-0.5235$

0.1703

0.3185

$-0.4055$

$-0.8142$

0.0032

0.0518

$-0.6200$

$-1.1691$

$-0.0709$

0.0269 
BMI (low versus normal)

Baseline mMRC $(m M R C \geq 2)$

Baseline SGRQ

Prior exacerbations ( $\geq 1$ versus 0 )

Number of patients $=4669$

Number of observations $=11,463$

$\mathrm{AIC}=8856.77$
0.3220

$-0.4203$

$-0.9795$

0.1390

0.1408

0.0046

$-0.0061$

0.0152

0.4013

0.2226

$-0.1104$

0.5557

0.1901

AIC, Akaike information criterion; BMI, body-mass index; CVD, cardiovascular disease; FEV ${ }_{1}$, forced expiratory volume in one second; mMRC, modified Medical Research Council score; SGRQ, St George's respiratory questionnaire. *Other, any reported non-CVD comorbidity. Bold $=P<0.05$. 
Table A5 Resource Utilization - Day and Night Home Visits

Explanatory Variable
Effect
95\% Confidence Intervals

Lower Upper

Intercept

Time, years

Recent moderate exacerbations

Recent severe exacerbations

$\mathrm{FEV}_{1} \%$ predicted (lagged 12 months)

Dyspnea symptoms (lagged 12 months) - most

days versus none

Dyspnea symptoms (lagged 12 months) - several

days versus none

Cough and sputum (lagged 12 months)

Age, years

CVD comorbidity (yes versus no)

Other comorbidity (yes versus no)*

Smoking status (current versus former)

Gender (female versus male)

BMI (high versus normal)
$-0.3437$

$-1.1905$

0.5031

0.4263

$-0.0562$

$-0.6063$

0.4940

0.8414

0.0297

$-0.0053$

0.0648

0.0965

0.1027

$-0.4805$

0.6859

0.7300

0.3729

$-0.3412$

1.0869

0.3061

$-0.1645$

$-0.7229$

0.3938

0.5635

$-0.1454$

$-0.7972$

0.5063

0.6618

$-0.7955$

$-1.7509$

0.1599

0.1027 
BMI (low versus normal)

Baseline mMRC $(m M R C \geq 2)$

Baseline SGRQ

Prior Exacerbations ( $\geq 1$ versus 0 )

Number of patients $=4669$

Number of observations $=11,463$

$\mathrm{AIC}=3025.99$
0.2669

0.9875

0.0127

1.3104

0.6188

2.0019

0.0002

AIC, Akaike information criterion; BMI, body-mass index; CVD, cardiovascular disease; $\mathrm{FEV}_{1}$, forced expiratory volume in one second; mMRC, modified Medical Research Council score; SGRQ, St George's respiratory questionnaire. *Other, any reported non-CVD comorbidity. Bold $=P<0.05$. Note: includes day and night time home visits by a health care worker. 
Table A6 Resource Utilization - Outpatient Physician Office Visits

Explanatory Variable
Effect
95\% Confidence Intervals

$P$ Value

\begin{tabular}{|c|c|c|c|c|}
\hline & & \multirow[b]{2}{*}{ Lower } & \multirow[b]{2}{*}{ Upper } & \\
\hline & & & & \\
\hline Intercept & -0.3691 & -1.1591 & 0.4209 & 0.3598 \\
\hline Time, years & 0.0531 & -0.0369 & 0.1430 & 0.2476 \\
\hline Recent moderate exacerbations & 0.1105 & 0.0981 & 0.1230 & $<0.0001$ \\
\hline Recent severe exacerbations & 0.0391 & -0.0129 & 0.0912 & 0.1405 \\
\hline $\mathrm{FEV}_{1} \%$ predicted (lagged 12 months) & -0.0108 & -0.0158 & -0.0058 & $<0.0001$ \\
\hline Dyspnea symptoms (lagged 12 months) - most & 0.6740 & 0.3144 & 1.0335 & 0.0002 \\
\hline Dyspnea symptom (lagged 12 months) - several & 0.4651 & 0.1161 & 0.8142 & 0.0090 \\
\hline Cough and sputum (lagged 12 months) & 0.1559 & 0.0074 & 0.3044 & 0.0396 \\
\hline Age, years & -0.0114 & -0.0201 & -0.0027 & 0.0100 \\
\hline CVD comorbidity (yes versus no) & -0.0645 & -0.2312 & 0.1022 & 0.4480 \\
\hline Other comorbidity (yes versus no)* & 0.0262 & -0.1691 & 0.2214 & 0.7928 \\
\hline Smoking status (current versus former) & -0.2163 & -0.3653 & -0.0673 & 0.0044 \\
\hline Gender (female versus male) & 0.1847 & 0.0287 & 0.3407 & 0.0203 \\
\hline BMI (high versus normal) & -0.2485 & -0.4379 & -0.0591 & 0.0101 \\
\hline
\end{tabular}


BMI (low versus normal)

Baseline mMRC $(\mathrm{mMRC} \geq 2)$

Baseline SGRQ

Prior Exacerbations ( $\geq 1$ versus 0$)$

Number of Patients $=4669$

Number of Observations $=11,463$

$\mathrm{AIC}=25,644.62$

$-0.2544 \quad-0.4428 \quad-0.0661$

0.0081

$-0.1588$

$-0.4185$

0.1009

0.2307

$-0.0016$

$-0.0063$

0.0030

0.4930

0.3351

0.1898

0.4805

$<0.0001$

AIC, Akaike information criterion; BMI, body-mass index; CVD, cardiovascular disease; $\mathrm{FEV}_{1}$, forced expiratory volume in one second; mMRC, modified Medical Research Council score; SGRQ, St George's respiratory questionnaire. *Other, any reported non-CVD comorbidity. 
Table A7 Resource Utilization - Hospital Outpatient Visits

\begin{tabular}{|c|c|c|c|c|}
\hline \multirow[t]{2}{*}{ Explanatory Variable } & \multirow[t]{2}{*}{ Effect } & \multicolumn{2}{|c|}{ 95\% Confidence Intervals } & \multirow[t]{2}{*}{$P$ Value } \\
\hline & & Lower & Upper & \\
\hline Intercept & -0.9087 & -2.1694 & 0.3519 & 0.1577 \\
\hline Time, years & -0.1898 & -0.3425 & -0.0371 & 0.0148 \\
\hline Recent moderate exacerbations & 0.0844 & 0.0589 & 0.1100 & $<0.0001$ \\
\hline Recent severe exacerbations & 0.0406 & -0.0485 & 0.1298 & 0.3715 \\
\hline $\mathrm{FEV}_{1} \%$ predicted (lagged 12 lonths) & -0.0123 & -0.0202 & -0.0044 & 0.0023 \\
\hline Dyspnea symptoms (lagged 12 months) - most & -0.576 & -1.0130 & -0.1390 & 0.0098 \\
\hline Dyspnea symptoms (lagged 12 months) - several & -0.1533 & -0.5544 & 0.2479 & 0.4539 \\
\hline Cough and sputum (lagged 12 months) & 0.1369 & -0.1030 & 0.3768 & 0.2635 \\
\hline Age, years & -0.0011 & -0.0156 & 0.0134 & 0.8816 \\
\hline CVD comorbidity (yes versus no) & 0.0388 & -0.2226 & 0.3003 & 0.7709 \\
\hline Other comorbidity (yes versus no)* & -0.2584 & -0.5279 & 0.0110 & 0.0601 \\
\hline Smoking status (current versus former) & -0.4398 & -0.6877 & -0.1918 & 0.0005 \\
\hline Gender (female versus male) & -0.6256 & -0.9357 & -0.3155 & $<0.0001$ \\
\hline BMI (high versus normal) & -0.6665 & -1.0561 & -0.2768 & 0.0008 \\
\hline
\end{tabular}


BMI (low versus normal)

Baseline mMRC $(\mathrm{mMRC} \geq 2)$

Baseline SGRQ

Prior Exacerbations ( $\geq 1$ versus 0$)$

Number of Patients $=4669$

Number of Observations $=11463$

$\mathrm{AIC}=22427.22$

$\begin{array}{llll}0.2183 & -0.0504 & 0.4869 & 0.1113\end{array}$

$-0.3818$

0.5769

0.6900

0.0140

0.0065

0.0214

0.0002

0.4280

0.1844

0.6717

0.0006

AIC, Akaike information criterion; BMI, body-mass index; CVD, cardiovascular disease; $\mathrm{FEV}_{1}$, forced expiratory volume in one second; mMRC, modified Medical Research Council score; SGRQ, St George's respiratory questionnaire. *Other, any reported non-CVD comorbidity. 
APPENDIX B Covariance matrices

Table A8 Covariance matrix (moderate exacerbations; fixed effects)

\begin{tabular}{|c|c|c|c|c|c|c|c|c|c|c|c|c|c|c|c|c|c|c|c|}
\hline & Intercept & $\begin{array}{l}\text { Study } \\
\text { years }\end{array}$ & $\begin{array}{c}\text { Time* } \\
\text { prior exac. }\end{array}$ & $\begin{array}{c}\text { Any } \mathbf{F E V}_{1} \\
\text { predicted }\end{array}$ & $\begin{array}{l}\text { Dyspnea } \\
\text { (lagged 12 } \\
\text { months), } \\
\text { most days }\end{array}$ & $\begin{array}{c}\text { Dyspnea } \\
\text { (lagged 12 } \\
\text { months), } \\
\text { several } \\
\text { days } \\
\end{array}$ & $\begin{array}{c}\text { Any } \\
\text { symptom }\end{array}$ & Age & $\begin{array}{l}\text { CVD co- } \\
\text { morbidity } \\
\text { (yes) }\end{array}$ & $\begin{array}{l}\text { Other co- } \\
\text { morbidity } \\
\text { (yes) }\end{array}$ & $\begin{array}{l}\text { Current } \\
\text { smoker }\end{array}$ & Female & $\begin{array}{l}\text { High BMI } \\
\text { category }\end{array}$ & $\begin{array}{l}\text { Low BMI } \\
\text { category }\end{array}$ & $\begin{array}{l}\text { Baseline } \\
\text { 6MWD }\end{array}$ & $\begin{array}{c}\text { Fibrin- } \\
\text { ogen }\end{array}$ & $\begin{array}{l}\text { Baseline } \\
\text { mMRC }\end{array}$ & $\begin{array}{c}\text { Baseline } \\
\text { SGRQ }\end{array}$ & Prior exac \\
\hline Intercept & 0.0526700 & -0.0040100 & 0.0038690 & -0.0000520 & -0.0027300 & -0.0030180 & 0.0003053 & -0.0003780 & 0.0004249 & -0.0000790 & -0.0012830 & -0.0008700 & -0.0005290 & -0.0013160 & -0.0000150 & -0.0000084 & -0.0003970 & -0.0000780 & -0.0088560 \\
\hline Study years & -0.0040100 & 0.0018570 & -0.0018560 & 0.0000015 & 0.0000401 & 0.0000467 & 0.0000196 & 0.0000003 & 0.0000093 & -0.0000032 & -0.0000073 & -0.0000140 & -0.0000029 & 0.0000240 & -0.0000001 & 0.0000000 & 0.0000069 & 0.0000001 & 0.0038940 \\
\hline Time*prior exac. & 0.0038690 & -0.0018560 & 0.0026590 & -0.0000001 & 0.0000112 & -0.0000470 & -0.0000240 & 0.0000004 & -0.0000220 & 0.0000044 & 0.0000136 & 0.0000090 & -0.0000140 & -0.0000370 & 0.0000000 & 0.0000000 & -0.0000003 & -0.0000001 & -0.0055300 \\
\hline $\begin{array}{l}\text { Any } \mathbf{F E V}_{1} \% \\
\text { predicted }\end{array}$ & -0.0000520 & 0.0000015 & -0.0000001 & 0.0000014 & 0.0000121 & 0.0000059 & -0.0000020 & -0.0000004 & -0.0000031 & -0.0000043 & -0.0000042 & -0.0000091 & -0.0000087 & 0.0000071 & 0.0000000 & 0.0000000 & 0.0000062 & 0.0000001 & 0.0000047 \\
\hline $\begin{array}{l}\text { Dyspnea (lagged } 12 \\
\text { months), most days }\end{array}$ & -0.0027300 & 0.0000401 & 0.0000112 & 0.0000121 & 0.0045110 & 0.0037940 & -0.0002440 & -0.0000093 & -0.0000430 & -0.0000560 & -0.0000280 & -0.0001220 & -0.0000540 & 0.0000276 & -0.0000005 & 0.0000002 & -0.0001020 & -0.0000160 & -0.0000680 \\
\hline $\begin{array}{l}\text { Dyspnea (lagged } 12 \\
\text { months), several }\end{array}$ & -0.0030180 & 0.0000467 & -0.0000470 & 0.0000059 & 0.0037940 & 0.0044850 & -0.0000570 & -0.0000044 & -0.0000570 & -0.0000720 & -0.0000820 & -0.0000740 & 0.0000341 & -0.0000100 & -0.0000006 & 0.0000002 & -0.0000400 & -0.0000080 & 0.0000150 \\
\hline Any symptom & 0.0003053 & 0.0000196 & -0.0000240 & -0.0000020 & -0.0002440 & -0.0000570 & 0.0011110 & -0.0000018 & 0.0000188 & -0.0000630 & -0.0002620 & 0.0001260 & 0.0000371 & -0.0000330 & -0.0000002 & -0.0000002 & 0.0000221 & -0.0000055 & -0.0000200 \\
\hline Age & -0.0003780 & 0.0000003 & 0.0000004 & -0.0000004 & -0.0000093 & -0.0000044 & -0.0000018 & 0.0000060 & -0.0000086 & -0.0000063 & 0.0000220 & 0.0000123 & 0.0000099 & 0.0000044 & 0.0000001 & -0.0000001 & -0.0000046 & 0.0000005 & 0.0000010 \\
\hline $\begin{array}{l}\text { CVD comorbidity } \\
\text { (yes) }\end{array}$ & 0.0004249 & 0.0000093 & -0.0000220 & -0.0000031 & -0.0000430 & -0.0000570 & 0.0000188 & -0.0000086 & 0.0012310 & -0.0001450 & 0.0000133 & 0.0001986 & -0.0000840 & -0.0000460 & 0.0000001 & 0.0000001 & -0.0000190 & -0.0000022 & 0.0000230 \\
\hline $\begin{array}{l}\begin{array}{l}\text { ther comorbidity } \\
\text { (yes) }\end{array} \\
\text { a }\end{array}$ & -0.0000790 & -0.0000032 & 0.0000044 & -0.0000043 & -0.0000560 & -0.0000720 & -0.0000630 & -0.0000063 & -0.0001450 & 0.0014140 & 0.0000499 & -0.0001830 & -0.0001230 & 0.0001169 & 0.0000001 & -0.0000001 & -0.0000490 & -0.0000025 & -0.0000210 \\
\hline Current smoker & -0.0012830 & -0.0000073 & 0.0000136 & -0.0000042 & -0.0000280 & -0.0000820 & -0.0002620 & 0.0000220 & 0.0000133 & 0.0000499 & 0.0012970 & 0.0000006 & 0.0001044 & -0.0001800 & -0.0000002 & -0.0000004 & 0.0000651 & -0.0000003 & 0.0000199 \\
\hline Female & -0.0008700 & -0.0000140 & 0.0000090 & -0.0000091 & -0.0001220 & -0.0000740 & 0.0001260 & 0.0000123 & 0.0001986 & -0.0001830 & 0.0000006 & 0.0011330 & 0.0001050 & -0.0001530 & 0.0000008 & -0.0000003 & -0.0000640 & 0.0000027 & -0.0001030 \\
\hline High BMI & -0.0005290 & -0.0000029 & -0.0000140 & -0.0000087 & -0.0000540 & 0.0000341 & 0.0000371 & 0.0000099 & -0.0000840 & -0.0001230 & 0.0001044 & 0.0001050 & 0.0016080 & 0.0002966 & 0.0000010 & -0.0000008 & -0.0000350 & -0.0000004 & 0.0000774 \\
\hline Low BMI & -0.0013160 & 0.0000240 & -0.0000370 & 0.0000071 & 0.0000276 & -0.0000100 & -0.0000330 & 0.0000044 & -0.0000460 & 0.0001169 & -0.0001800 & -0.0001530 & 0.0002966 & 0.0021680 & 0.0000002 & 0.0000007 & 0.0000760 & -0.0000018 & 0.0000486 \\
\hline Baseline 6MWD & -0.0000150 & -0.0000001 & 0.0000000 & 0.0000000 & -0.0000005 & -0.0000006 & -0.0000002 & 0.0000001 & 0.0000001 & 0.0000001 & -0.0000002 & 0.0000008 & 0.0000010 & 0.0000002 & 0.0000000 & 0.0000000 & 0.0000012 & 0.0000000 & 0.0000000 \\
\hline Fibrinogen & -0.0000084 & 0.0000000 & 0.0000000 & 0.0000000 & 0.0000002 & 0.0000002 & -0.0000002 & -0.0000001 & 0.0000001 & -0.0000001 & -0.0000004 & -0.0000003 & -0.0000008 & 0.0000007 & 0.0000000 & 0.0000000 & 0.0000000 & 0.0000000 & -0.0000003 \\
\hline Baseline mMRC & -0.0003970 & 0.0000069 & -0.0000003 & 0.0000062 & -0.0001020 & -0.0000400 & 0.0000221 & -0.0000046 & -0.0000190 & -0.0000490 & 0.0000651 & -0.0000640 & -0.0000350 & 0.0000760 & 0.0000012 & 0.0000000 & 0.0014800 & -0.0000140 & -0.0000420 \\
\hline Baseline SGRQ & -0.0000780 & 0.0000001 & -0.0000001 & 0.0000001 & -0.0000160 & -0.0000080 & -0.0000055 & 0.0000005 & -0.0000022 & -0.0000025 & -0.0000003 & 0.0000027 & -0.0000004 & -0.0000018 & 0.0000000 & 0.0000000 & -0.0000140 & 0.0000012 & -0.0000038 \\
\hline Prior exac. & -0.0088560 & 0.0038940 & -0.0055300 & 0.0000047 & -0.0000680 & 0.0000150 & -0.0000200 & 0.0000010 & 0.0000230 & -0.0000210 & 0.0000199 & -0.0001030 & 0.0000774 & 0.0000486 & 0.0000000 & -0.0000003 & -0.0000420 & -0.0000038 & 0.0127100 \\
\hline
\end{tabular}

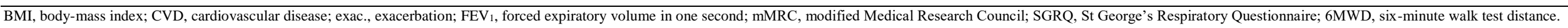


Table A9 Covariance matrix for (severe exacerbations; fixed effects)

\begin{tabular}{|c|c|c|c|c|c|c|c|c|c|c|c|c|c|c|c|c|c|c|c|}
\hline & Intercept & $\begin{array}{l}\text { Study } \\
\text { years }\end{array}$ & $\begin{array}{l}\text { Time*" } \\
\text { prior } \\
\text { exac. }\end{array}$ & $\begin{array}{c}\text { Any } \\
\text { FEV } \\
\text { predicted }^{2}\end{array}$ & $\begin{array}{c}\text { Dyspnea } \\
\text { (lagged } \\
12 \\
\text { months, } \\
\text { most days }\end{array}$ & $\begin{array}{c}\text { Dyspnea } \\
\text { (lagged } \\
12 \\
\text { months), } \\
\text { several } \\
\text { days } \\
\end{array}$ & $\begin{array}{c}\text { Any } \\
\text { symptom }\end{array}$ & Age & $\begin{array}{l}\text { CVD co- } \\
\text { morbidity } \\
\text { (yes) }\end{array}$ & $\begin{array}{l}\text { Other co- } \\
\text { morbidity } \\
\text { (yes) }\end{array}$ & $\begin{array}{l}\text { Current } \\
\text { smoker }\end{array}$ & Female & $\begin{array}{c}\text { High } \\
\text { BMI } \\
\text { category }\end{array}$ & $\begin{array}{l}\text { Low BMI } \\
\text { category }\end{array}$ & 6MWD & $\begin{array}{c}\text { Fibrin- } \\
\text { ogen }\end{array}$ & $\begin{array}{l}\text { Baseline } \\
\text { mMRC }\end{array}$ & $\begin{array}{l}\text { Baseline } \\
\text { SGRQ }\end{array}$ & $\begin{array}{l}\text { Prior } \\
\text { exac. }\end{array}$ \\
\hline Intercept & 0.2247400 & -0.0175600 & 0.0168900 & -0.0001880 & -0.0150100 & -0.0161400 & 0.0010630 & -0.0015930 & 0.0015130 & -0.0003440 & -0.0053640 & -0.0039950 & -0.0016840 & -0.0047160 & -0.0000560 & -0.0000310 & -0.0020840 & -0.0003280 & -0.0411900 \\
\hline Study years & -0.0175600 & 0.0076740 & -0.0076680 & 0.0000068 & 0.0001416 & 0.0002067 & 0.0001327 & 0.0000001 & 0.0000169 & 0.0000033 & -0.0000470 & -0.0000700 & -0.0000620 & 0.0000609 & -0.0000008 & -0.0000001 & 0.0000135 & -0.0000004 & 0.0173800 \\
\hline Time* prior exac. & 0.0168900 & -0.0076680 & 0.0107900 & 0.0000002 & 0.0001356 & -0.0000910 & -0.0002170 & 0.0000031 & -0.0001180 & 0.0000089 & 0.0000976 & 0.0000258 & 0.0000252 & -0.0001210 & 0.0000004 & 0.0000003 & 0.0000092 & 0.0000005 & -0.0239800 \\
\hline $\begin{array}{l}\mathrm{Any} \mathrm{FEV}_{1} \% \\
\text { predicted }\end{array}$ & -0.0001880 & 0.0000068 & 0.0000002 & 0.0000067 & 0.0000526 & 0.0000280 & -0.0000076 & -0.0000020 & -0.0000170 & -0.0000130 & -0.0000160 & -0.0000450 & -0.0000390 & 0.0000258 & -0.0000002 & 0.0000001 & 0.0000257 & 0.0000002 & 0.0000173 \\
\hline $\begin{array}{l}\text { Dyspnea (lagged } \\
12 \text { months), most } \\
\text { days }\end{array}$ & -0.0150100 & 0.0001416 & 0.0001356 & 0.0000526 & 0.0200500 & 0.0176500 & -0.0009830 & -0.0000200 & -0.0001420 & -0.0002930 & -0.0000850 & -0.0003720 & -0.0000150 & -0.0001180 & -0.0000010 & 0.0000004 & -0.0002710 & -0.0000510 & -0.0003410 \\
\hline $\begin{array}{l}\text { Dyspnea (lagged } \\
12 \text { month), } \\
\text { several }\end{array}$ & -0.0161400 & 0.0002067 & -0.0000910 & 0.0000280 & 0.0176500 & 0.0212800 & -0.0001910 & -0.0000059 & -0.0001190 & -0.0004130 & -0.0002590 & -0.0002110 & 0.0002245 & -0.0003160 & -0.0000016 & 0.0000005 & -0.0000840 & -0.0000240 & 0.0000596 \\
\hline Any symptom & 0.0010630 & 0.0001327 & -0.0002170 & -0.0000076 & -0.0009830 & -0.0001910 & 0.0044290 & -0.0000070 & -0.0000004 & -0.0002240 & -0.0010430 & 0.0004606 & 0.0000862 & -0.0001250 & -0.0000007 & -0.0000008 & 0.0000853 & -0.0000210 & 0.0001418 \\
\hline Age & -0.0015930 & 0.0000001 & 0.0000031 & -0.0000020 & -0.0000200 & -0.0000059 & -0.0000070 & 0.0000247 & -0.0000290 & -0.0000250 & 0.0000844 & 0.0000578 & 0.0000327 & 0.0000207 & 0.0000002 & -0.0000002 & -0.0000210 & 0.0000017 & 0.0000041 \\
\hline $\begin{array}{l}\text { CVD co- } \\
\text { morbidity (yes) }\end{array}$ & 0.0015130 & 0.0000169 & -0.0001180 & -0.0000170 & -0.0001420 & -0.0001190 & -0.0000004 & -0.0000290 & 0.0043170 & -0.0005070 & 0.0001206 & 0.0007453 & -0.0002300 & -0.0001660 & 0.0000007 & 0.0000002 & -0.0001610 & -0.0000083 & 0.0001474 \\
\hline $\begin{array}{l}\text { Other co- } \\
\text { morbidity (yes) }\end{array}$ & -0.0003440 & 0.0000033 & 0.0000089 & -0.0000130 & -0.0002930 & -0.0004130 & -0.0002240 & -0.0000250 & -0.0005070 & 0.0052340 & 0.0000754 & -0.0007720 & -0.0005840 & 0.0003829 & 0.0000003 & 0.0000000 & -0.0002360 & -0.0000080 & -0.0000710 \\
\hline Current smoker & -0.0053640 & -0.0000470 & 0.0000976 & -0.0000160 & -0.0000850 & -0.0002590 & -0.0010430 & 0.0000844 & 0.0001206 & 0.0000754 & 0.0048930 & 0.0000833 & 0.0004544 & -0.0005350 & -0.0000004 & -0.0000011 & 0.0003857 & -0.0000025 & 0.0001153 \\
\hline Female & -0.0039950 & -0.0000700 & 0.0000258 & -0.0000450 & -0.0003720 & -0.0002110 & 0.0004606 & 0.0000578 & 0.0007453 & -0.0007720 & 0.0000833 & 0.0049020 & 0.0003239 & -0.0006610 & 0.0000032 & -0.0000008 & -0.0002630 & 0.0000108 & -0.0003600 \\
\hline High BMI & -0.0016840 & -0.0000620 & 0.0000252 & -0.0000390 & -0.0000150 & 0.0002245 & 0.0000862 & 0.0000327 & -0.0002300 & -0.0005840 & 0.0004544 & 0.0003239 & 0.0067820 & 0.0012630 & 0.0000032 & -0.0000024 & -0.0000180 & -0.0000033 & 0.0000671 \\
\hline Low BMI & -0.0047160 & 0.0000609 & -0.0001210 & 0.0000258 & -0.0001180 & -0.0003160 & -0.0001250 & 0.0000207 & -0.0001660 & 0.0003829 & -0.0005350 & -0.0006610 & 0.0012630 & 0.0065920 & 0.0000003 & 0.0000026 & 0.0003608 & -0.0000050 & 0.0000159 \\
\hline 6MWD & -0.0000560 & -0.0000008 & 0.0000004 & -0.0000002 & -0.0000010 & -0.0000016 & -0.0000007 & 0.0000002 & 0.0000007 & 0.0000003 & -0.0000004 & 0.0000032 & 0.0000032 & 0.0000003 & 0.0000001 & 0.0000000 & 0.0000050 & 0.0000002 & -0.0000010 \\
\hline Fibrinogen & -0.0000310 & -0.0000001 & 0.0000003 & 0.0000001 & 0.0000004 & 0.0000005 & -0.0000008 & -0.0000002 & 0.0000002 & 0.0000000 & -0.0000011 & -0.0000008 & -0.0000024 & 0.0000026 & 0.0000000 & 0.0000001 & -0.0000001 & 0.0000000 & -0.0000011 \\
\hline Baseline mMRC & -0.0020840 & 0.0000135 & 0.0000092 & 0.0000257 & -0.0002710 & -0.0000840 & 0.0000853 & -0.0000210 & -0.0001610 & -0.0002360 & 0.0003857 & -0.0002630 & -0.0000180 & 0.0003608 & 0.0000050 & -0.0000001 & 0.0061180 & -0.0000490 & -0.0003410 \\
\hline Baseline SGRQ & -0.0003280 & -0.0000004 & 0.0000005 & 0.0000002 & -0.0000510 & -0.0000240 & -0.0000210 & 0.0000017 & -0.0000083 & -0.0000080 & -0.0000025 & 0.0000108 & -0.0000033 & -0.0000050 & 0.0000002 & 0.0000000 & -0.0000490 & 0.0000046 & -0.0000190 \\
\hline Prior exac. & -0.0411900 & 0.0173800 & -0.0239800 & 0.0000173 & -0.0003410 & 0.0000596 & 0.0001418 & 0.0000041 & 0.0001474 & -0.0000710 & 0.0001153 & -0.0003600 & 0.0000671 & 0.0000159 & -0.0000010 & -0.0000011 & -0.0003410 & -0.0000190 & 0.0581200 \\
\hline
\end{tabular}


Table A10 Covariance matrix ( $\mathrm{FEV}_{1}$; fixed effects)

\begin{tabular}{|c|c|c|c|c|c|c|c|c|c|c|c|c|c|c|c|c|c|c|}
\hline & Intercept & $\begin{array}{l}\text { Study } \\
\text { years }\end{array}$ & $\begin{array}{c}\text { Time* } \\
\text { smoker }\end{array}$ & $\begin{array}{l}\text { Moderate } \\
\text { exac. }\end{array}$ & $\begin{array}{l}\text { Severe } \\
\text { exac. }\end{array}$ & Height & Age & $\begin{array}{c}\text { CVD co- } \\
\text { morbidity } \\
\text { (yes) }\end{array}$ & $\begin{array}{c}\text { Other co- } \\
\text { morbidity } \\
\text { (yes) }\end{array}$ & $\begin{array}{l}\text { Current } \\
\text { smoker }\end{array}$ & Female & High BMI & Low BMI & $\begin{array}{l}\text { Baseline } \\
\text { 6MWD }\end{array}$ & $\begin{array}{c}\text { Fibrin- } \\
\text { ogen }\end{array}$ & $\begin{array}{l}\text { Baseline } \\
\text { mMRC }\end{array}$ & $\begin{array}{l}\text { Baseline } \\
\text { SGRQ }\end{array}$ & Prior exac. \\
\hline Intercept & 82182. & -21.1117000 & 19.304400 & -8.4971000 & -3.1467000 & $\begin{array}{r}364.530000 \\
0\end{array}$ & $\begin{array}{r}195.130000 \\
0\end{array}$ & -7.3696000 & -233.19000 & -612.36000 & -4811.2100 & -272.74000 & -370.5400 & -5.4084000 & -3.6456000 & -158.77000 & -36.9438000 & -302.14000 \\
\hline Study years & -21.11170 & 9.9497000 & -9.9098000 & 0.0059500 & -0.5218000 & -0.0005000 & 0.0082350 & 0.0934100 & -0.0206700 & 20.6269000 & -0.1832000 & -0.0461500 & 0.0117100 & -0.0013900 & 0.0010710 & 0.0684000 & 0.0021770 & 0.1704000 \\
\hline Time*smoker & 19.3044000 & -9.9098000 & 28.663300 & 0.6580000 & -0.6502000 & 0.0050310 & 0.0166200 & -0.0766400 & 0.0107400 & -59.3219000 & 0.2148000 & 0.1170000 & 0.3585000 & -0.0011700 & -0.0021300 & -0.2924000 & 0.0110700 & -0.5572000 \\
\hline Moderate exac. & -8.497100 & 0.0059500 & 0.6580000 & 5.3156000 & 1.1220000 & 0.0576000 & 0.0013130 & 0.7139000 & -0.3620000 & -0.5647000 & -0.0679800 & 0.8369000 & 0.4332000 & -0.0007600 & -0.0058400 & -0.3035000 & -0.0404600 & -4.2729000 \\
\hline Severe exac. & -3.146700 & -0.5218000 & -0.6502000 & 1.1220000 & 18.069300 & 0.0582900 & -0.0354300 & -0.3745000 & 0.3194000 & 1.8311000 & 0.8986000 & 1.4770000 & -1.5820000 & 0.0037330 & -0.0097800 & -0.1642000 & -0.0959100 & -3.4465000 \\
\hline Height & -364.5300 & -0.0005000 & 0.0050310 & 0.0576000 & 0.0582900 & 1.9854000 & 0.2632000 & 0.6210000 & 0.7577000 & 0.2111000 & 23.9684000 & -0.3289000 & 0.7476000 & -0.0067900 & 0.0029780 & 0.5416000 & 0.0531900 & 1.0033000 \\
\hline Age & -195.1300 & 0.0082350 & 0.0166200 & 0.0013130 & -0.0354300 & 0.2632000 & 2.2090000 & -3.3801000 & -2.6328000 & 6.9994000 & 6.6143000 & 3.3859000 & 0.6074000 & 0.0232600 & -0.0181000 & -1.1492000 & 0.1564000 & 1.1859000 \\
\hline $\begin{array}{l}\text { CVD co- } \\
\text { morbidity (yes) }\end{array}$ & -7.369600 & 0.0934100 & -0.0766400 & 0.7139000 & -0.3745000 & 0.6210000 & -3.3801000 & 451.48000 & -71.3392000 & -6.4798000 & 69.2478000 & -34.9238000 & -0.9924000 & 0.0606500 & 0.0219200 & -1.0110000 & -0.4525000 & -2.4974000 \\
\hline $\begin{array}{l}\text { Other co- } \\
\text { morbidity (yes) }\end{array}$ & -233.1900 & -0.0206700 & 0.0107400 & -0.3620000 & 0.3194000 & 0.7577000 & -2.6328000 & -71.3392000 & 515.08000 & 4.9674000 & -70.9318000 & -68.9301000 & 56.6916000 & -0.0112400 & 0.0092280 & 0.4984000 & -1.0762000 & -0.4765000 \\
\hline Current smoker & $\begin{array}{r}612.360000 \\
0\end{array}$ & 20.6269000 & -59.321900 & -0.5647000 & 1.8311000 & 0.2111000 & 6.9994000 & -6.4798000 & 4.9674000 & 565.32000 & 2.6388000 & 38.6468000 & -79.5795000 & -0.0653600 & -0.1023000 & 35.4339000 & -0.4423000 & 19.9507000 \\
\hline Female & -4811.2100 & -0.1832000 & 0.2148000 & -0.0679800 & 0.8986000 & 23.9684000 & 6.6143000 & 69.2478000 & -70.9318000 & 2.6388000 & $\begin{array}{r}726.280000 \\
0\end{array}$ & 22.7179000 & -40.2152000 & 0.1387000 & -0.0626500 & 8.8505000 & 1.7299000 & -9.9670000 \\
\hline High BMI & -272.7400 & -0.0461500 & 0.1170000 & 0.8369000 & 1.4770000 & -0.3289000 & 3.3859000 & -34.9238000 & -68.9301000 & 38.646800 & 22.7179000 & 590.97000 & 126.34000 & 0.3013000 & -0.2722000 & -8.1444000 & 0.0691300 & 28.2300000 \\
\hline Low BMI & -370.5400 & 0.0117100 & 0.3585000 & 0.4332000 & -1.5820000 & 0.7476000 & 0.6074000 & -0.9924000 & 56.6916000 & -79.5795000 & -40.2152000 & 126.340000 & 835.390000 & 0.0511600 & 0.2225000 & 18.8557000 & -1.2035000 & -25.0944000 \\
\hline Baseline 6MWD & -5.408400 & -0.0013900 & -0.0011700 & -0.0007600 & 0.0037330 & -0.0067900 & 0.0232600 & 0.0606500 & -0.0112400 & -0.0653600 & 0.1387000 & 0.3013000 & 0.0511600 & 0.0093660 & 0.0007610 & 0.4658000 & 0.0167300 & 0.0614200 \\
\hline Fibrinogen & -3.645600 & 0.0010710 & -0.0021300 & -0.0058400 & -0.0097800 & 0.0029780 & -0.0181000 & 0.0219200 & 0.0092280 & -0.1023000 & -0.0626500 & -0.2722000 & 0.2225000 & 0.0007610 & 0.0096210 & -0.0578000 & -0.0049400 & -0.1207000 \\
\hline Baseline mMRC & -158.7700 & 0.0684000 & -0.2924000 & -0.3035000 & -0.1642000 & 0.5416000 & -1.1492000 & -1.0110000 & 0.4984000 & 35.4339000 & 8.8505000 & -8.1444000 & 18.8557000 & 0.4658000 & -0.0578000 & $\begin{array}{r}558.810000 \\
0\end{array}$ & -6.1569000 & -23.2089000 \\
\hline Baseline SGRQ & -36.94380 & 0.0021770 & 0.0110700 & -0.0404600 & -0.0959100 & 0.0531900 & 0.1564000 & -0.4525000 & -1.0762000 & -0.4423000 & 1.7299000 & 0.0691300 & -1.2035000 & 0.0167300 & -0.0049400 & -6.1569000 & 0.3796000 & -1.7660000 \\
\hline Prior exac. & -302.1400 & 0.1704000 & -0.5572000 & -4.2729000 & -3.4465000 & 1.0033000 & 1.1859000 & -2.4974000 & -0.4765000 & 19.9507000 & -9.9670000 & 28.2300000 & -25.0944000 & 0.0614200 & -0.1207000 & -23.2089000 & -1.7660000 & 403.290000 \\
\hline
\end{tabular}


Table A11 Covariance matrix (dyspnea, most versus none or several days; fixed effects)

\begin{tabular}{|c|c|c|c|c|c|c|c|c|c|c|c|c|c|c|c|}
\hline & Intercept & Study years & $\begin{array}{l}\text { FEV }_{1} \% \\
\text { predicted }\end{array}$ & Age & $\begin{array}{c}\text { CVD co- } \\
\text { morbidity } \\
\text { (yes) }\end{array}$ & $\begin{array}{l}\text { Other co- } \\
\text { morbidity } \\
\text { (yes) }\end{array}$ & $\begin{array}{l}\text { Current } \\
\text { smoker }\end{array}$ & Female & $\begin{array}{l}\text { High BMI } \\
\text { category }\end{array}$ & $\begin{array}{l}\text { Low BMI } \\
\text { category }\end{array}$ & $\begin{array}{l}\text { Baseline } \\
\text { 6MWD }\end{array}$ & Fibrinogen & $\begin{array}{l}\text { MMRC } \\
\text { GOLD }\end{array}$ & $\begin{array}{c}\text { Baseline } \\
\text { SGRQ }\end{array}$ & Prior exac. \\
\hline Intercept & 0.7879000 & -0.0114100 & -0.0008700 & -0.0068600 & 0.0074680 & 0.0003550 & -0.0225400 & -0.0149200 & -0.0119300 & -0.0169600 & -0.0002800 & -0.0001700 & -0.0058500 & -0.0015500 & -0.0098300 \\
\hline Study years & -0.0114100 & 0.0043470 & 0.0000093 & 0.0000110 & -0.0000600 & 0.0000100 & 0.0000037 & 0.0000074 & 0.0000180 & 0.0001120 & -0.0000012 & 0.0000009 & 0.0000490 & 0.0000140 & 0.0001260 \\
\hline $\begin{array}{l}\mathrm{FEV}_{1} \% \\
\text { predicted }\end{array}$ & -0.0008700 & 0.0000093 & 0.0000230 & -0.0000034 & -0.0000500 & -0.0001000 & -0.0000400 & -0.0001200 & -0.0001200 & 0.0001110 & -0.0000003 & 0.0000004 & 0.0000700 & -0.0000005 & 0.0000610 \\
\hline Age & -0.0068600 & 0.0000110 & -0.0000034 & 0.0001040 & -0.0001500 & -0.0001200 & 0.0003240 & 0.0001930 & 0.0001840 & 0.0000030 & 0.0000012 & -0.0000010 & -0.0000700 & 0.0000081 & 0.0000500 \\
\hline $\begin{array}{l}\text { CVD co- } \\
\text { morbidity (yes) }\end{array}$ & 0.0074680 & -0.0000600 & -0.0000500 & -0.0001500 & 0.0214000 & -0.0033300 & -0.0002500 & 0.0033350 & -0.0012900 & -0.0001100 & 0.0000036 & 0.0000000 & -0.0002100 & -0.0000400 & -0.0003500 \\
\hline $\begin{array}{l}\text { Other co- } \\
\text { morbidity (yes) }\end{array}$ & 0.0003550 & 0.0000100 & -0.0001000 & -0.0001200 & -0.0033300 & 0.0246400 & 0.0002980 & -0.0034500 & -0.0027800 & 0.0020490 & -0.0000005 & -0.0000015 & -0.0000700 & -0.0000400 & -0.0002300 \\
\hline Current smoker & -0.0225400 & 0.0000037 & -0.0000400 & 0.0003240 & -0.0002500 & 0.0002980 & 0.0209000 & 0.0001470 & 0.0023790 & -0.0040900 & -0.0000014 & -0.0000062 & 0.0013720 & -0.0000300 & 0.0008190 \\
\hline Female & -0.0149200 & 0.0000074 & -0.0001200 & 0.0001930 & 0.0033350 & -0.0034500 & 0.0001470 & 0.0212500 & 0.0022060 & -0.0029400 & 0.0000110 & -0.0000076 & -0.0003900 & 0.0000530 & -0.0013100 \\
\hline High BMI & -0.0119300 & 0.0000180 & -0.0001200 & 0.0001840 & -0.0012900 & -0.0027800 & 0.0023790 & 0.0022060 & 0.0282700 & 0.0052260 & 0.0000160 & -0.0000100 & -0.0007400 & -0.0000005 & 0.0012650 \\
\hline Low BMI & -0.0169600 & 0.0001120 & 0.0001110 & 0.0000030 & -0.0001100 & 0.0020490 & -0.0040900 & -0.0029400 & 0.0052260 & 0.0400600 & 0.0000003 & 0.0000150 & 0.0011020 & -0.0000400 & -0.0008000 \\
\hline Baseline 6MWD & -0.0002800 & -0.0000012 & -0.0000003 & 0.0000012 & 0.0000036 & -0.0000005 & -0.0000014 & 0.0000110 & 0.0000160 & 0.0000003 & 0.0000004 & 0.0000000 & 0.0000190 & 0.0000006 & 0.0000024 \\
\hline Fibrinogen & -0.0001700 & 0.0000009 & 0.0000004 & -0.0000010 & 0.0000000 & -0.0000015 & -0.0000062 & -0.0000076 & -0.0000100 & 0.0000150 & 0.0000000 & 0.0000005 & 0.0000001 & -0.0000001 & -0.0000050 \\
\hline mMRC & -0.0058500 & 0.0000490 & 0.0000700 & -0.0000700 & -0.0002100 & -0.0000700 & 0.0013720 & -0.0003900 & -0.0007400 & 0.0011020 & 0.0000190 & 0.0000001 & 0.0252500 & -0.0002600 & -0.0006300 \\
\hline Baseline SGRQ & -0.0015500 & 0.0000140 & -0.0000005 & 0.0000081 & -0.0000400 & -0.0000400 & -0.0000300 & 0.0000530 & -0.0000005 & -0.0000400 & 0.0000006 & -0.0000001 & -0.0002600 & 0.0000230 & -0.0000700 \\
\hline Prior exac. & -0.0098300 & 0.0001260 & 0.0000610 & 0.0000500 & -0.0003500 & -0.0002300 & 0.0008190 & -0.0013100 & 0.0012650 & -0.0008000 & 0.0000024 & -0.0000050 & -0.0006300 & -0.0000700 & 0.0189000 \\
\hline
\end{tabular}


Table A12 Covariance matrix (dyspnea,(none vs most or several days; fixed effects)

\begin{tabular}{|c|c|c|c|c|c|c|c|c|c|c|c|c|c|c|c|}
\hline & Intercept & Study years & $\begin{array}{l}\mathrm{FEV}_{1} \% \\
\text { predicted }\end{array}$ & Age & $\begin{array}{c}\text { CVD co- } \\
\text { morbidity } \\
\text { (yes) }\end{array}$ & $\begin{array}{l}\text { Other co- } \\
\text { morbidity } \\
\text { (yes) }\end{array}$ & $\begin{array}{l}\text { Current } \\
\text { smoker }\end{array}$ & Female & High BMI & $\begin{array}{l}\text { Low BMI } \\
\text { category }\end{array}$ & $\begin{array}{l}\text { Baseline } \\
\text { 6MWD }\end{array}$ & Fibrinogen & $\begin{array}{l}\text { MMRC } \\
\text { GOLD }\end{array}$ & $\begin{array}{l}\text { Baseline } \\
\text { SGRQ }\end{array}$ & Prior exac. \\
\hline Intercept & 1.5051000 & -0.0223400 & -0.0021300 & -0.0128800 & 0.0180800 & 0.0093590 & -0.0483000 & -0.0240300 & -0.0239300 & -0.0315700 & -0.0005600 & -0.0003500 & -0.0075100 & -0.0027600 & -0.0160400 \\
\hline Study years & -0.0223400 & 0.0089490 & 0.0000190 & 0.0000150 & 0.0000870 & -0.0001700 & -0.0000300 & 0.0001750 & 0.0001920 & 0.0003180 & -0.0000014 & 0.0000017 & 0.0001300 & 0.0000150 & 0.0002620 \\
\hline $\begin{array}{l}\mathrm{FEV}_{1} \% \\
\text { predicted }\end{array}$ & -0.0021300 & 0.0000190 & 0.0000400 & -0.0000028 & -0.0000700 & -0.0001700 & -0.0000500 & -0.0001700 & -0.0001700 & 0.0001690 & -0.0000007 & 0.0000008 & 0.0001640 & 0.0000025 & 0.0001300 \\
\hline Age & -0.0128800 & 0.0000150 & -0.0000028 & 0.0001940 & -0.0003700 & -0.0003000 & 0.0006460 & 0.0003000 & 0.0003570 & 0.0000170 & 0.0000026 & -0.0000019 & -0.0001800 & 0.0000180 & 0.0000430 \\
\hline $\begin{array}{l}\text { CVD co- } \\
\text { morbidity (yes) }\end{array}$ & 0.0180800 & 0.0000870 & -0.0000700 & -0.0003700 & 0.0425400 & -0.0074500 & -0.0018700 & 0.0069940 & -0.0020000 & 0.0005560 & 0.0000050 & 0.0000003 & -0.0008300 & -0.0000600 & -0.0002900 \\
\hline $\begin{array}{l}\text { Other co- } \\
\text { morbidity (yes) }\end{array}$ & 0.0093590 & -0.0001700 & -0.0001700 & -0.0003000 & -0.0074500 & 0.0447300 & 0.0003590 & -0.0069100 & -0.0062700 & 0.0049250 & 0.0000001 & -0.0000029 & 0.0009360 & -0.0001400 & -0.0006500 \\
\hline Current smoker & -0.0483000 & -0.0000300 & -0.0000500 & 0.0006460 & -0.0018700 & 0.0003590 & 0.0397800 & -0.0004200 & 0.0039290 & -0.0083700 & -0.0000007 & -0.0000089 & 0.0016810 & 0.0000110 & 0.0009710 \\
\hline Female & -0.0240300 & 0.0001750 & -0.0001700 & 0.0003000 & 0.0069940 & -0.0069100 & -0.0004200 & 0.0398900 & 0.0043140 & -0.0056600 & 0.0000220 & -0.0000200 & -0.0004300 & 0.0000580 & -0.0028600 \\
\hline High BMI & -0.0239300 & 0.0001920 & -0.0001700 & 0.0003570 & -0.0020000 & -0.0062700 & 0.0039290 & 0.0043140 & 0.0550300 & 0.0090430 & 0.0000350 & -0.0000300 & -0.0012700 & -0.0000200 & 0.0023230 \\
\hline Low BMI & -0.0315700 & 0.0003180 & 0.0001690 & 0.0000170 & 0.0005560 & 0.0049250 & -0.0083700 & -0.0056600 & 0.0090430 & 0.0906800 & -0.0000032 & 0.0000290 & -0.0003200 & -0.0000300 & -0.0008300 \\
\hline Baseline 6MWD & -0.0005600 & -0.0000014 & -0.0000007 & 0.0000026 & 0.0000050 & 0.0000001 & -0.0000007 & 0.0000220 & 0.0000350 & -0.0000032 & 0.0000009 & 0.0000000 & 0.0000390 & 0.0000012 & 0.0000065 \\
\hline Fibrinogen & -0.0003500 & 0.0000017 & 0.0000008 & -0.0000019 & 0.0000003 & -0.0000029 & -0.0000089 & -0.0000200 & -0.0000300 & 0.0000290 & 0.0000000 & 0.0000010 & -0.0000035 & -0.0000001 & -0.0000100 \\
\hline mMRC & -0.0075100 & 0.0001300 & 0.0001640 & -0.0001800 & -0.0008300 & 0.0009360 & 0.0016810 & -0.0004300 & -0.0012700 & -0.0003200 & 0.0000390 & -0.0000035 & 0.0525700 & -0.0006000 & -0.0006600 \\
\hline Baseline SGRQ & -0.0027600 & 0.0000150 & 0.0000025 & 0.0000180 & -0.0000600 & -0.0001400 & 0.0000110 & 0.0000580 & -0.0000200 & -0.0000300 & 0.0000012 & -0.0000001 & -0.0006000 & 0.0000430 & -0.0001400 \\
\hline Prior exac. & -0.0160400 & 0.0002620 & 0.0001300 & 0.0000430 & -0.0002900 & -0.0006500 & 0.0009710 & -0.0028600 & 0.0023230 & -0.0008300 & 0.0000065 & -0.0000100 & -0.0006600 & -0.0001400 & 0.0371300 \\
\hline
\end{tabular}


Table A13 Covariance matrix (cough and sputum; fixed effects)

\begin{tabular}{|c|c|c|c|c|c|c|c|c|c|c|c|c|c|c|c|}
\hline & Intercept & Study years & $\begin{array}{l}\text { FEV }_{1} \% \\
\text { predicted }\end{array}$ & Age & $\begin{array}{c}\text { CVD co- } \\
\text { morbidity } \\
\text { (yes) }\end{array}$ & $\begin{array}{l}\text { Other co- } \\
\text { morbidity } \\
\text { (yes) }\end{array}$ & $\begin{array}{l}\text { Current } \\
\text { smoker }\end{array}$ & Female & High BMI & Low BMI & $\begin{array}{l}\text { Baseline } \\
\text { 6MWD }\end{array}$ & Fibrinogen & $\begin{array}{l}\text { MMRC } \\
\text { GOLD }\end{array}$ & $\begin{array}{c}\text { Baseline } \\
\text { SGRQ }\end{array}$ & Prior exac. \\
\hline Intercept & 0.8995000 & -0.0106800 & -0.0010000 & -0.0078700 & 0.0120800 & -0.0027000 & -0.0344400 & -0.0125300 & -0.0115500 & -0.0198100 & -0.0003200 & -0.0001900 & -0.0074200 & -0.0017500 & -0.0113300 \\
\hline Study years & -0.0106800 & 0.0042290 & 0.0000180 & 0.0000051 & -0.0000300 & -0.0000500 & -0.0000600 & -0.0000300 & -0.0000200 & 0.0001050 & -0.0000013 & 0.0000009 & 0.0000310 & 0.0000038 & 0.0000800 \\
\hline $\begin{array}{l}\mathrm{FEV}_{1} \% \\
\text { predicted }\end{array}$ & -0.0010000 & 0.0000180 & 0.0000230 & -0.0000035 & -0.0000400 & -0.0000900 & -0.0000800 & -0.0001100 & -0.0001200 & 0.0001210 & -0.0000005 & 0.0000005 & 0.0000900 & 0.0000027 & 0.0000660 \\
\hline Age & -0.0078700 & 0.0000051 & -0.0000035 & 0.0001190 & -0.0002000 & -0.0001300 & 0.0004230 & 0.0001840 & 0.0002120 & 0.0000230 & 0.0000013 & -0.0000011 & -0.0000800 & 0.0000083 & 0.0000480 \\
\hline $\begin{array}{l}\text { CVD co- } \\
\text { morbidity (yes) }\end{array}$ & 0.0120800 & -0.0000300 & -0.0000400 & -0.0002000 & 0.0250500 & -0.0042300 & -0.0022400 & 0.0044650 & -0.0012700 & -0.0005600 & 0.0000046 & -0.0000006 & -0.0002800 & -0.0000700 & -0.0009500 \\
\hline $\begin{array}{l}\text { Other co- } \\
\text { morbidity (yes) }\end{array}$ & -0.0027000 & -0.0000500 & -0.0000900 & -0.0001300 & -0.0042300 & 0.0285300 & 0.0020180 & -0.0047100 & -0.0039100 & 0.0027170 & -0.0000006 & -0.0000001 & -0.0003400 & -0.0000500 & 0.0001740 \\
\hline Current smoker & -0.0344400 & -0.0000600 & -0.0000800 & 0.0004230 & -0.0022400 & 0.0020180 & 0.0284200 & -0.0020500 & 0.0012160 & -0.0039000 & -0.0000045 & -0.0000052 & 0.0019890 & 0.0000660 & 0.0021570 \\
\hline Female & -0.0125300 & -0.0000300 & -0.0001100 & 0.0001840 & 0.0044650 & -0.0047100 & -0.0020500 & 0.0248200 & 0.0028160 & -0.0035300 & 0.0000150 & -0.0000088 & -0.0007000 & 0.0000072 & -0.0021400 \\
\hline High BMI & -0.0115500 & -0.0000200 & -0.0001200 & 0.0002120 & -0.0012700 & -0.0039100 & 0.0012160 & 0.0028160 & 0.0328700 & 0.0059290 & 0.0000200 & -0.0000200 & -0.0009800 & -0.0000300 & 0.0007520 \\
\hline Low BMI & -0.0198100 & 0.0001050 & 0.0001210 & 0.0000230 & -0.0005600 & 0.0027170 & -0.0039000 & -0.0035300 & 0.0059290 & 0.0453900 & -0.0000011 & 0.0000160 & 0.0010590 & -0.0000400 & -0.0007000 \\
\hline Baseline 6MWD & -0.0003200 & -0.0000013 & -0.0000005 & 0.0000013 & 0.0000046 & -0.0000006 & -0.0000045 & 0.0000150 & 0.0000200 & -0.0000011 & 0.0000005 & 0.0000000 & 0.0000230 & 0.0000008 & 0.0000014 \\
\hline Fibrinogen & -0.0001900 & 0.0000009 & 0.0000005 & -0.0000011 & -0.0000006 & -0.0000001 & -0.0000052 & -0.0000088 & -0.0000200 & 0.0000160 & 0.0000000 & 0.0000005 & -0.0000014 & -0.0000002 & -0.0000045 \\
\hline mMRC & -0.0074200 & 0.0000310 & 0.0000900 & -0.0000800 & -0.0002800 & -0.0003400 & 0.0019890 & -0.0007000 & -0.0009800 & 0.0010590 & 0.0000230 & -0.0000014 & 0.0299100 & -0.0003100 & -0.0006000 \\
\hline Baseline SGRQ & -0.0017500 & 0.0000038 & 0.0000027 & 0.0000083 & -0.0000700 & -0.0000500 & 0.0000660 & 0.0000072 & -0.0000300 & -0.0000400 & 0.0000008 & -0.0000002 & -0.0003100 & 0.0000220 & -0.0000700 \\
\hline Prior exac. & -0.0113300 & 0.0000800 & 0.0000660 & 0.0000480 & -0.0009500 & 0.0001740 & 0.0021570 & -0.0021400 & 0.0007520 & -0.0007000 & 0.0000014 & -0.0000045 & -0.0006000 & -0.0000700 & 0.0217700 \\
\hline
\end{tabular}


Table A14 Covariance matrix (6MWD; fixed effects)

\begin{tabular}{|c|c|c|c|c|c|c|c|c|c|c|c|c|c|c|c|c|c|c|c|}
\hline & Intercept & $\begin{array}{l}\text { Study } \\
\text { years }\end{array}$ & $\begin{array}{l}\text { Moderate } \\
\text { exac. }\end{array}$ & $\begin{array}{l}\text { Severe } \\
\text { exac. }\end{array}$ & $\begin{array}{l}\mathrm{FEV}_{1} \% \\
\text { predicted }\end{array}$ & $\begin{array}{c}\text { Dyspnea } \\
\text { (lagged } \\
12 \\
\text { months), } \\
\text { most days }\end{array}$ & $\begin{array}{c}\text { Dyspnea } \\
\text { (lagged } \\
12 \\
12 \\
\text { months), } \\
\text { several } \\
\text { days } \\
\end{array}$ & $\underset{\text { symptom }}{\text { Any }}$ & Age & $\begin{array}{l}\text { CVD co- } \\
\text { morbidity } \\
\text { (yes) }\end{array}$ & $\begin{array}{c}\text { Other co- } \\
\text { morbidity } \\
\text { (yes) }\end{array}$ & $\begin{array}{l}\text { Current } \\
\text { smoker }\end{array}$ & Female & $\begin{array}{c}\text { High } \\
\text { BMI } \\
\text { category }\end{array}$ & $\begin{array}{c}\text { Low BMI } \\
\text { category }\end{array}$ & $\begin{array}{c}\text { Fibrinoge } \\
\mathbf{n}\end{array}$ & $\begin{array}{l}\text { Baseline } \\
\text { mMRC }\end{array}$ & $\begin{array}{l}\text { Baseline } \\
\text { SGRQ }\end{array}$ & $\begin{array}{l}\text { Prior } \\
\text { exac. }\end{array}$ \\
\hline Intercept & 775.57000 & -8.1279000 & -0.8200000 & 0.3437000 & -1.4927000 & -14.07190 & -15.073700 & 1.6746000 & -8.0683000 & 13.1251000 & 1.8159000 & -32.627900 & 10.1131000 & -0.8853000 & -24.723600 & -0.1920000 & 8.7820000 & -1.2086000 & -7.5818000 \\
\hline Study years & -8.1279000 & 2.5871000 & 0.1544000 & -0.1592000 & 0.0211600 & 0.3901000 & 0.2552000 & 0.2683000 & 0.0050860 & 0.0218600 & -0.1065000 & -0.0052400 & -0.0765400 & 0.0233600 & 0.1484000 & 0.0007810 & 0.1915000 & 0.0035600 & 0.0071740 \\
\hline Moderate exac. & -0.8200000 & 0.1544000 & 1.7882000 & 0.2656000 & 0.0142200 & -0.1438000 & -0.0326500 & -0.1156000 & -0.0023300 & 0.1664000 & -0.1218000 & 0.2943000 & -0.4142000 & 0.2204000 & 0.1569000 & -0.0014000 & -0.0301800 & -0.0071600 & -1.3698000 \\
\hline Severe exac. & 0.3437000 & -0.1592000 & 0.2656000 & 7.1219000 & 0.0450000 & 0.2924000 & 0.1839000 & -0.1609000 & -0.0242500 & -0.1106000 & -0.0251800 & 0.0031960 & -0.1446000 & 0.2738000 & -0.3601000 & -0.0016500 & 0.1315000 & -0.0270900 & -1.0229000 \\
\hline $\begin{array}{l}\mathrm{FEV}_{1} \% \\
\text { predicted }\end{array}$ & -1.4927000 & 0.0211600 & 0.0142200 & 0.0450000 & 0.0237500 & 0.1165000 & 0.0609200 & -0.0047300 & -0.0021000 & -0.0533500 & -0.0918200 & -0.0432500 & -0.1223000 & -0.1121000 & 0.1122000 & 0.0005130 & 0.1192000 & 0.0032210 & 0.0581000 \\
\hline $\begin{array}{l}\text { Dyspnea (lagged } \\
12 \text { months), most } \\
\text { days } \\
\text { Dyspnea (lagged }\end{array}$ & -14.071900 & 0.3901000 & -0.1438000 & 0.2924000 & 0.1165000 & 30.709500 & 21.9915000 & -2.1325000 & -0.1107000 & -0.0093100 & -0.3952000 & -0.1609000 & -0.9340000 & -0.7825000 & 0.0805900 & 0.0023850 & -0.6171000 & -0.1662000 & -0.5665000 \\
\hline $\begin{array}{l}\text { Dyspnea (lagged } \\
12 \text { months), } \\
\text { several }\end{array}$ & -15.073700 & 0.2552000 & -0.0326500 & 0.1839000 & 0.0609200 & 21.99150 & 26.5686000 & -0.5228000 & -0.0741700 & -0.2457000 & -0.1420000 & -0.8161000 & -0.7779000 & -0.1035000 & -0.1144000 & 0.0026770 & -0.0218100 & -0.0895000 & -0.5971000 \\
\hline Any symptom & 1.6746000 & 0.2683000 & -0.1156000 & -0.1609000 & -0.0047300 & -2.1325000 & -0.5228000 & 12.0476000 & -0.0356200 & 0.1295000 & -0.3038000 & -3.0651000 & 1.0620000 & 0.4315000 & -0.0724200 & -0.0013800 & 0.4068000 & -0.0535000 & -0.4823000 \\
\hline Age & -8.0683000 & 0.0050860 & -0.0023300 & -0.0242500 & -0.0021000 & -0.1107000 & -0.0741700 & -0.0356200 & 0.1347000 & -0.2215000 & -0.2008000 & 0.4602000 & 0.2160000 & 0.1751000 & 0.0747600 & -0.0013800 & -0.1400000 & 0.0079430 & 0.0537600 \\
\hline $\begin{array}{l}\text { CVD co- } \\
\text { morbidity (yes) }\end{array}$ & 13.125100 & 0.0218600 & 0.1664000 & -0.1106000 & -0.0533500 & -0.0093100 & -0.2457000 & 0.1295000 & -0.2215000 & 29.0187000 & -4.4768000 & -0.3791000 & 4.3891000 & -1.7880000 & -0.5230000 & -0.0010500 & -0.3387000 & -0.0506000 & -0.8083000 \\
\hline $\begin{array}{l}\text { Other co- } \\
\text { morbidity (yes) }\end{array}$ & 1.8159000 & -0.1065000 & -0.1218000 & -0.0251800 & -0.0918200 & -0.3952000 & -0.1420000 & -0.3038000 & -0.2008000 & -4.4768000 & 32.751400 & 0.5922000 & -4.6456000 & -3.9144000 & 3.1410000 & -0.0004900 & -0.5080000 & -0.0826500 & -0.2466000 \\
\hline Current smoker & -32.627900 & -0.0052400 & 0.2943000 & 0.0031960 & -0.0432500 & -0.1609000 & -0.8161000 & -3.0651000 & 0.4602000 & -0.3791000 & 0.5922000 & 28.8038000 & -0.2382000 & 2.8981000 & -5.0347000 & -0.0086900 & 2.0561000 & -0.0119600 & 0.8870000 \\
\hline Female & -10.113100 & -0.0765400 & -0.4142000 & -0.1446000 & -0.1223000 & -0.9340000 & -0.7779000 & 1.0620000 & 0.2160000 & 4.3891000 & -4.6456000 & -0.2382000 & 28.0869000 & 1.9795000 & -3.3595000 & -0.0098600 & -1.2498000 & 0.0303500 & -1.5562000 \\
\hline High BMI & -0.8853000 & 0.0233600 & 0.2204000 & 0.2738000 & -0.1121000 & -0.7825000 & -0.1035000 & 0.4315000 & 0.1751000 & -1.7880000 & -3.9144000 & 2.8981000 & 1.9795000 & 36.850400 & 7.1900000 & -0.0215600 & -1.9559000 & -0.0433500 & 0.9466000 \\
\hline Low BMI & -24.72360 & 0.1484000 & 0.1569000 & -0.3601000 & 0.1122000 & 0.0805900 & -0.1144000 & -0.0724200 & 0.0747600 & -0.5230000 & 3.1410000 & -5.0347000 & -3.3595000 & 7.1900000 & 54.9424000 & 0.0185300 & 1.1167000 & -0.0506500 & -1.2836000 \\
\hline Fibrinogen & -0.1920000 & 0.0007810 & -0.0014000 & -0.0016500 & 0.0005130 & 0.0023850 & 0.0026770 & -0.0013800 & -0.0013800 & -0.0010500 & -0.0004900 & -0.0086900 & -0.0098600 & -0.0215600 & 0.0185300 & 0.0006140 & -0.0041800 & -0.0002900 & -0.0052300 \\
\hline Baseline mMRC & 8.7820000 & 0.1915000 & -0.0301800 & 0.1315000 & 0.1192000 & -0.6171000 & -0.0218100 & 0.4068000 & -0.1400000 & -0.3387000 & -0.5080000 & 2.0561000 & -1.2498000 & -1.9559000 & 1.1167000 & -0.0041800 & 34.123300 & -0.4139000 & -1.0117000 \\
\hline Baseline SGRQ & -1.2086000 & 0.0035600 & -0.0071600 & -0.0270900 & 0.0032210 & -0.1662000 & -0.0895000 & -0.0535000 & 0.0079430 & -0.0506000 & -0.0826500 & -0.0119600 & 0.0303500 & -0.0433500 & -0.0506500 & -0.0002900 & -0.4139000 & 0.0242600 & -0.1063000 \\
\hline Prior exac. & -7.5818000 & 0.0071740 & -1.3698000 & -1.0229000 & 0.0581000 & -0.5665000 & -0.5971000 & -0.4823000 & 0.0537600 & -0.8083000 & -0.2466000 & 0.8870000 & -1.5562000 & 0.9466000 & -1.2836000 & -0.0052300 & -1.0117000 & -0.1063000 & 26.6820000 \\
\hline
\end{tabular}


Table A15 Covariance matrix (SGRQ; fixed effects)

\begin{tabular}{|c|c|c|c|c|c|c|c|c|c|c|c|c|c|c|c|c|c|c|c|c|}
\hline & Intercept & $\begin{array}{l}\text { Study } \\
\text { years }\end{array}$ & $\begin{array}{l}\text { Moderat } \\
\text { e exac. }\end{array}$ & $\begin{array}{l}\text { Severe } \\
\text { exac. }\end{array}$ & 6MWD & $\begin{array}{l}\mathrm{FEV}_{1} \% \\
\text { predicted }\end{array}$ & $\begin{array}{c}\text { Dyspnea } \\
\text { (lagged } \\
12 \\
\text { months), } \\
\text { most } \\
\text { days } \\
\end{array}$ & $\begin{array}{c}\text { Dyspnea } \\
\text { (lagged } \\
12 \\
\text { months), } \\
\text { several } \\
\text { days } \\
\end{array}$ & $\begin{array}{c}\text { Any } \\
\text { symptom }\end{array}$ & Age & $\begin{array}{l}\text { CVD co- } \\
\text { morbidit } \\
\text { y (yes) }\end{array}$ & $\begin{array}{c}\text { Other } \\
\text { co- } \\
\text { morbidit } \\
\text { y (yes) }\end{array}$ & $\begin{array}{l}\text { Current } \\
\text { smoker }\end{array}$ & Female & $\begin{array}{c}\text { High } \\
\text { BMI } \\
\text { category }\end{array}$ & $\begin{array}{c}\text { Low } \\
\text { BMI } \\
\text { category }\end{array}$ & $\begin{array}{l}\text { Baseline } \\
\text { 6MWD }\end{array}$ & $\begin{array}{l}\text { Fibrin- } \\
\text { ogen }\end{array}$ & $\begin{array}{l}\text { Baseline } \\
\text { mMRC }\end{array}$ & $\begin{array}{l}\text { Prior } \\
\text { exac. }\end{array}$ \\
\hline Intercept & 15.3825000 & -0.1509000 & -0.0308200 & -0.0523300 & -0.0018500 & -0.0008800 & -0.5054000 & -0.3500000 & 0.0164300 & -0.1392000 & 0.1036000 & -0.0577600 & -0.5141000 & -0.5780000 & -0.1809000 & -0.4960000 & -0.0030800 & -0.0038600 & -0.6183000 & -0.3254000 \\
\hline Study years & -0.1509000 & 0.0470200 & 0.0030410 & -0.0023300 & 0.0000580 & 0.0000100 & -0.0016200 & -0.0010000 & 0.0003620 & 0.0003100 & 0.0003530 & -0.0007200 & 0.0017910 & 0.0030710 & 0.0000340 & 0.0038210 & -0.0000600 & 0.0000190 & 0.0047310 & 0.0015730 \\
\hline Moderate exac. & -0.0308200 & 0.0030410 & 0.0295400 & 0.0038390 & 0.0000076 & 0.0000095 & -0.0059200 & -0.0034000 & -0.0036900 & 0.0001800 & 0.0023250 & -0.0030100 & 0.0053290 & -0.0023200 & 0.0034100 & 0.0032640 & -0.0000100 & -0.0000200 & -0.0017700 & -0.0228200 \\
\hline Severe exac. & -0.0523300 & -0.0023300 & 0.0038390 & 0.1036000 & 0.0000930 & 0.0000160 & -0.0041100 & 0.0015590 & 0.0034550 & 0.0002100 & -0.0023900 & -0.0022900 & -0.0007200 & 0.0089510 & 0.0074520 & -0.0073100 & -0.0000300 & -0.0000400 & -0.0011700 & -0.0165800 \\
\hline 6MWD & -0.0018500 & 0.0000580 & 0.0000076 & 0.0000930 & 0.0000071 & -0.0000003 & 0.0001360 & 0.0000320 & 0.0000033 & 0.0000100 & 0.0000690 & 0.0000090 & 0.0001010 & -0.0000500 & 0.0001470 & 0.0000840 & -0.0000044 & 0.0000003 & 0.0001740 & 0.0000680 \\
\hline $\begin{array}{l}\mathrm{FEV}_{1} \% \\
\text { predicted }\end{array}$ & -0.0008800 & 0.0000100 & 0.0000095 & 0.0000160 & -0.0000003 & 0.0000004 & 0.0000730 & 0.0000300 & -0.0000011 & 0.0000039 & -0.0000200 & -0.0000300 & -0.0000200 & 0.0001240 & -0.0000700 & 0.0000670 & -0.0000002 & 0.0000003 & 0.0000560 & 0.0000360 \\
\hline $\begin{array}{l}12 \text { months), most } \\
\text { days }\end{array}$ & -0.5054000 & -0.0016200 & -0.0059200 & -0.0041100 & 0.0001360 & 0.0000730 & 0.5222000 & 0.3738000 & -0.0444500 & 0.0008210 & 0.0021880 & -0.0236700 & 0.0013510 & 0.0255100 & -0.0169700 & -0.0134600 & -0.0000500 & -0.0000300 & -0.0476200 & -0.0074400 \\
\hline $\begin{array}{l}\text { Dyspnea (lagged } \\
\text { 12 months), } \\
\text { several }\end{array}$ & -0.3500000 & -0.0010000 & -0.0034000 & 0.0015590 & 0.0000320 & 0.0000300 & 0.3738000 & 0.4590000 & -0.0110000 & 0.0000640 & -0.0003300 & -0.0142700 & -0.0086700 & 0.0102300 & -0.0084200 & -0.0173700 & -0.0000300 & -0.0000098 & -0.0270000 & -0.0082000 \\
\hline Any symptom & 0.0164300 & 0.0003620 & -0.0036900 & 0.0034550 & 0.0000033 & -0.0000011 & -0.0444500 & -0.0110000 & 0.1988000 & -0.0005800 & 0.0074560 & -0.0083700 & -0.0476100 & 0.0184900 & 0.0096160 & -0.0059300 & 0.0000073 & -0.0000500 & -0.0089200 & -0.0108000 \\
\hline Age & -0.1392000 & 0.0003100 & 0.0001800 & 0.0002100 & 0.0000100 & 0.0000039 & 0.0008210 & 0.0000640 & -0.0005800 & 0.0020440 & -0.0033600 & -0.0026600 & 0.0068350 & 0.0040260 & 0.0025420 & 0.0021350 & 0.0000055 & -0.0000100 & 0.0020590 & 0.0021180 \\
\hline $\begin{array}{l}\text { CVD co- } \\
\text { morbidity (yes) }\end{array}$ & 0.1036000 & 0.0003530 & 0.0023250 & -0.0023900 & 0.0000690 & -0.0000200 & 0.0021880 & -0.0003300 & 0.0074560 & -0.0033600 & 0.4237000 & -0.0671500 & -0.0078800 & 0.0578000 & -0.0259500 & -0.0075600 & 0.0000360 & -0.0000047 & -0.0098200 & -0.0094000 \\
\hline $\begin{array}{l}\text { Other co- } \\
\text { morbidity (yes) }\end{array}$ & -0.0577600 & -0.0007200 & -0.0030100 & -0.0022900 & 0.0000090 & -0.0000300 & -0.0236700 & -0.0142700 & -0.0083700 & -0.0026600 & -0.0671500 & 0.4802000 & 0.0074010 & -0.0846500 & -0.0603000 & 0.0414000 & 0.0000470 & -0.0000100 & -0.0198200 & -0.0053300 \\
\hline Current smoker & -0.5141000 & 0.0017910 & 0.0053290 & -0.0007200 & 0.0001010 & -0.0000200 & 0.0013510 & -0.0086700 & -0.0476100 & 0.0068350 & -0.0078800 & 0.0074010 & 0.4268000 & -0.0098900 & 0.0412400 & -0.0731900 & -0.0000900 & -0.0001100 & 0.0287200 & 0.0142000 \\
\hline Female & -0.5780000 & 0.0030710 & -0.0023200 & 0.0089510 & -0.0000500 & 0.0001240 & 0.0255100 & 0.0102300 & 0.0184900 & 0.0040260 & 0.0578000 & -0.0846500 & -0.0098900 & 0.4454000 & 0.0031690 & -0.0268300 & 0.0000790 & -0.0000089 & 0.0309700 & -0.0018100 \\
\hline High BMI & -0.1809000 & 0.0000340 & 0.0034100 & 0.0074520 & 0.0001470 & -0.0000700 & -0.0169700 & -0.0084200 & 0.0096160 & 0.0025420 & -0.0259500 & -0.0603000 & 0.0412400 & 0.0031690 & 0.5633000 & 0.1077000 & 0.0002430 & -0.0003000 & -0.0127800 & 0.0171200 \\
\hline Low BMI & -0.4960000 & 0.0038210 & 0.0032640 & -0.0073100 & 0.0000840 & 0.0000670 & -0.0134600 & -0.0173700 & -0.0059300 & 0.0021350 & -0.0075600 & 0.0414000 & -0.0731900 & -0.0268300 & 0.1077000 & 0.7943000 & -0.0000200 & 0.0002810 & 0.0139900 & -0.0196400 \\
\hline Baseline 6MWD & -0.0030800 & -0.0000600 & -0.0000100 & -0.0000300 & -0.0000044 & -0.0000002 & -0.0000500 & -0.0000300 & 0.0000073 & 0.0000055 & 0.0000360 & 0.0000470 & -0.0000900 & 0.0000790 & 0.0002430 & -0.0000200 & 0.0000110 & 0.0000004 & 0.0005000 & 0.0000630 \\
\hline Fibrinogen & -0.0038600 & 0.0000190 & -0.0000200 & -0.0000400 & 0.0000003 & 0.0000003 & -0.0000300 & -0.0000098 & -0.0000500 & -0.0000100 & -0.0000047 & -0.0000100 & -0.0001100 & -0.0000089 & -0.0003000 & 0.0002810 & 0.0000004 & 0.0000092 & -0.0000500 & -0.0000700 \\
\hline Baseline mMRC & -0.6183000 & 0.0047310 & -0.0017700 & -0.0011700 & 0.0001740 & 0.0000560 & -0.0476200 & -0.0270000 & -0.0089200 & 0.0020590 & -0.0098200 & -0.0198200 & 0.0287200 & 0.0309700 & -0.0127800 & 0.0139900 & 0.0005000 & -0.0000500 & 0.4499000 & -0.0329000 \\
\hline Prior exac. & -0.3254000 & 0.0015730 & -0.0228200 & -0.0165800 & 0.0000680 & 0.0000360 & -0.0074400 & -0.0082000 & -0.0108000 & 0.0021180 & -0.0094000 & -0.0053300 & 0.0142000 & -0.0018100 & 0.0171200 & -0.0196400 & 0.0000630 & -0.0000700 & -0.0329000 & 0.3934000 \\
\hline
\end{tabular}


Table A16 Covariance matrix (mortality; fixed effects)

\begin{tabular}{|c|c|c|c|c|c|c|c|c|c|c|c|c|c|c|c|c|c|}
\hline & $\begin{array}{c}\text { Moderate } \\
\text { exac. }\end{array}$ & $\begin{array}{l}\text { Severe } \\
\text { exac. }\end{array}$ & $\begin{array}{c}\text { GMWD } \\
\text { augmented }\end{array}$ & $\begin{array}{l}\mathrm{FEV}_{1} \% \\
\text { predicted }\end{array}$ & $\begin{array}{c}\text { Dyspnea } \\
\text { (lagged 12 } \\
\text { months), } \\
\text { most days }\end{array}$ & $\begin{array}{c}\text { Dyspnea } \\
\text { (lagged 12 } \\
\text { months), } \\
\text { several days }\end{array}$ & $\begin{array}{l}\text { Symptom } \\
\text { most days }\end{array}$ & Age & $\begin{array}{c}\text { CVD co- } \\
\text { morbidity } \\
\text { (yes) }\end{array}$ & $\begin{array}{c}\text { Other co- } \\
\text { morbidity } \\
\text { (yes) }\end{array}$ & $\begin{array}{l}\text { Current } \\
\text { smoker }\end{array}$ & Female & $\begin{array}{l}\text { High BMI } \\
\text { category }\end{array}$ & $\begin{array}{l}\text { Low BMI } \\
\text { category }\end{array}$ & Fibrinogen & $\begin{array}{l}\text { Baseline } \\
\text { mMRC }\end{array}$ & $\begin{array}{l}\text { Baseline } \\
\text { SGRQ }\end{array}$ \\
\hline Moderate exac. & 0.0037852 & -0.0009536 & -0.0000004 & 0.0000214 & -0.0004498 & -0.0005837 & -0.0003527 & 0.0000310 & 0.0002794 & -0.0003335 & 0.0005495 & -0.0006595 & 0.0004177 & 0.0001522 & -0.0000025 & -0.0004116 & -0.0000333 \\
\hline $\begin{array}{l}\text { 6MWD } \\
\text { augmented }\end{array}$ & -0.0000004 & 0.0000064 & 0.0000006 & -0.0000013 & -0.0000227 & -0.0000196 & 0.0000040 & 0.0000016 & 0.0000096 & 0.0000044 & -0.0000004 & 0.0000186 & 0.0000240 & -0.0000014 & 0.0000000 & 0.0000267 & 0.0000011 \\
\hline $\begin{array}{l}\text { FEV }_{1} \% \\
\text { predicted } \\
\text { Dyspnea }\end{array}$ & 0.0000214 & 0.0000494 & -0.0000013 & 0.0000364 & 0.0002654 & 0.0001542 & -0.0000687 & -0.0000113 & -0.0000885 & -0.0000744 & -0.0000956 & -0.0002339 & -0.0002044 & 0.0001396 & 0.0000005 & 0.0001059 & 0.0000023 \\
\hline $\begin{array}{l}\text { (lagged 12 } \\
\text { months), most } \\
\text { days } \\
\text { Dyspnea }\end{array}$ & -0.0004498 & 0.0000921 & -0.0000227 & 0.0002654 & 0.0993131 & 0.0825069 & -0.0052756 & -0.0000633 & -0.0004006 & -0.0005589 & 0.0001624 & -0.0019112 & -0.0024726 & -0.0023400 & -0.0000013 & -0.0029721 & -0.0004801 \\
\hline $\begin{array}{l}\text { (lagged 12 } \\
\text { months), } \\
\text { several }\end{array}$ & -0.0005837 & 0.0005358 & -0.0000196 & 0.0001542 & 0.0825069 & 0.1007333 & -0.0028780 & -0.0001023 & -0.0003788 & -0.0013102 & -0.0004381 & -0.0004569 & -0.0022597 & -0.0045485 & -0.0000013 & -0.0020880 & -0.0002572 \\
\hline $\begin{array}{l}\text { Symptom } \\
\text { most days }\end{array}$ & -0.0003527 & 0.0001759 & 0.0000040 & -0.0000687 & -0.0052756 & -0.0028780 & 0.0254577 & -0.0000664 & 0.0013234 & -0.0013912 & -0.0052671 & 0.0022173 & 0.0025811 & -0.0001866 & -0.0000048 & 0.0009641 & -0.0001546 \\
\hline Age & 0.0000310 & -0.0000507 & 0.0000016 & -0.0000113 & -0.0000633 & -0.0001023 & -0.0000664 & 0.0001572 & -0.0001580 & -0.0001465 & 0.0004001 & 0.0002588 & 0.0002198 & 0.0000321 & -0.0000006 & -0.0001185 & 0.0000093 \\
\hline $\begin{array}{l}\text { CVD co- } \\
\text { morbidity (yes) }\end{array}$ & 0.0002794 & 0.0008694 & 0.0000096 & -0.0000885 & -0.0004006 & -0.0003788 & 0.0013234 & -0.0001580 & 0.0232562 & -0.0029193 & -0.0000084 & 0.0036315 & -0.0009330 & 0.0000515 & -0.0000017 & -0.0001970 & -0.0000555 \\
\hline $\begin{array}{l}\text { Other co- } \\
\text { morbidity (yes) }\end{array}$ & -0.0003335 & -0.0006272 & 0.0000044 & -0.0000744 & -0.0005589 & -0.0013102 & -0.0013912 & -0.0001465 & -0.0029193 & 0.0333684 & 0.0009624 & -0.0043212 & -0.0036254 & 0.0018382 & -0.0000008 & -0.0003483 & -0.0000359 \\
\hline $\begin{array}{l}\text { Current } \\
\text { smoker }\end{array}$ & 0.0005495 & 0.0002450 & -0.0000004 & -0.0000956 & 0.0001624 & -0.0004381 & -0.0052671 & 0.0004001 & -0.0000084 & 0.0009624 & 0.0270082 & 0.0009413 & 0.0022005 & -0.0034808 & -0.0000024 & 0.0024520 & -0.0000037 \\
\hline Female & -0.0006595 & 0.0001818 & 0.0000186 & -0.0002339 & -0.0019112 & -0.0004569 & 0.0022173 & 0.0002588 & 0.0036315 & -0.0043212 & 0.0009413 & 0.0294044 & 0.0015925 & -0.0043518 & -0.0000067 & 0.0000986 & 0.0000113 \\
\hline $\begin{array}{l}\text { High BMI } \\
\text { category }\end{array}$ & 0.0004177 & 0.0011632 & 0.0000240 & -0.0002044 & -0.0024726 & -0.0022597 & 0.0025811 & 0.0002198 & -0.0009330 & -0.0036254 & 0.0022005 & 0.0015925 & 0.0348637 & 0.0080983 & -0.0000099 & 0.0000580 & -0.0000084 \\
\hline $\begin{array}{l}\text { Low BMI } \\
\text { category }\end{array}$ & 0.0001522 & -0.0001085 & -0.0000014 & 0.0001396 & -0.0023400 & -0.0045485 & -0.0001866 & 0.0000321 & 0.0000515 & 0.0018382 & -0.0034808 & -0.0043518 & 0.0080983 & 0.0397087 & 0.0000151 & 0.0017781 & -0.0000455 \\
\hline Fibrinogen & -0.0000025 & -0.0000010 & 0.0000000 & 0.0000005 & -0.0000013 & -0.0000013 & -0.0000048 & -0.0000006 & -0.0000017 & -0.0000008 & -0.0000024 & -0.0000067 & -0.0000099 & 0.0000151 & 0.0000004 & -0.0000027 & -0.0000002 \\
\hline $\begin{array}{l}\text { Baseline } \\
\text { mMRC }\end{array}$ & -0.0004116 & 0.0017171 & 0.0000267 & 0.0001059 & -0.0029721 & -0.0020880 & 0.0009641 & -0.0001185 & -0.0001970 & -0.0003483 & 0.0024520 & 0.0000986 & 0.0000580 & 0.0017781 & -0.0000027 & 0.0360665 & -0.0003247 \\
\hline $\begin{array}{l}\text { Baseline } \\
\text { SGRQ }\end{array}$ & -0.0000333 & -0.0000537 & 0.0000011 & 0.0000023 & -0.0004801 & -0.0002572 & -0.0001546 & 0.0000093 & -0.0000555 & -0.0000359 & -0.0000037 & 0.0000113 & -0.0000084 & -0.0000455 & -0.0000002 & -0.0003247 & 0.0000286 \\
\hline
\end{tabular}


Table A17 Covariance matrix for survival (fixed effects)

\begin{tabular}{|c|c|c|c|c|c|c|c|c|c|c|c|c|c|c|c|c|c|c|c|}
\hline & Intercept & $\begin{array}{c}\text { Moderate } \\
\text { exac. }\end{array}$ & $\begin{array}{l}\text { Severe } \\
\text { exac. }\end{array}$ & $\begin{array}{c}\text { 6MWD } \\
\text { augmente } \\
d\end{array}$ & $\begin{array}{l}\mathrm{FEV}_{1} \% \\
\text { predicted }\end{array}$ & $\begin{array}{c}\text { Dyspnea } \\
\text { most days }\end{array}$ & $\begin{array}{c}\text { Dyspnea } \\
\text { (lagged 12 } \\
\text { months), } \\
\text { several } \\
\text { days } \\
\end{array}$ & $\begin{array}{c}\text { Symptoms } \\
\text { most days }\end{array}$ & Age & $\begin{array}{c}\text { CVD co- } \\
\text { morbidity } \\
\text { (yes) }\end{array}$ & $\begin{array}{c}\text { Other co- } \\
\text { morbidity } \\
\text { (yes) }\end{array}$ & $\begin{array}{l}\text { Current } \\
\text { smoker }\end{array}$ & Female & $\begin{array}{l}\text { High BMI } \\
\text { category }\end{array}$ & $\begin{array}{l}\text { Low BMI } \\
\text { category }\end{array}$ & $\begin{array}{c}\text { Fibrinoge } \\
n\end{array}$ & $\begin{array}{l}\text { Baseline } \\
\text { mMRC }\end{array}$ & $\begin{array}{l}\text { Baseline } \\
\text { SGRQ }\end{array}$ & Scale \\
\hline Intercept & 0.7445900 & -0.0014810 & -0.0008500 & -0.0001700 & -0.0003460 & -0.0330840 & -0.0302390 & 0.0066220 & -0.0070450 & -0.0008520 & -0.0075890 & -0.0209270 & -0.0064020 & -0.0105250 & -0.0171820 & -0.0001370 & -0.0048730 & -0.0010570 & 0.0148100 \\
\hline $\begin{array}{l}\text { Moderate } \\
\text { exac. }\end{array}$ & -0.0014810 & 0.0022350 & -0.0011570 & -0.0000003 & 0.0000127 & -0.0002340 & -0.0003090 & -0.0002830 & 0.0000188 & 0.0001320 & -0.0002220 & 0.0003450 & -0.0003680 & 0.0002110 & 0.0001830 & -0.0000012 & -0.0002190 & -0.0000185 & -0.0000354 \\
\hline Severe exac. & -0.0008500 & -0.0011570 & 0.0112520 & 0.0000042 & 0.0000289 & 0.0001590 & 0.0003350 & 0.0006050 & -0.0000193 & 0.0004530 & -0.0003470 & 0.0000299 & 0.0001660 & 0.0006880 & -0.0005650 & -0.0000017 & 0.0003520 & -0.0000380 & 0.0000219 \\
\hline $\begin{array}{l}\text { 6MWD } \\
\text { augmented }\end{array}$ & -0.0001700 & -0.0000003 & 0.0000042 & 0.0000004 & -0.0000006 & -0.0000131 & -0.0000102 & 0.0000026 & 0.0000005 & 0.0000025 & 0.0000027 & -0.0000025 & 0.0000134 & 0.0000131 & -0.0000033 & 0.0000000 & 0.0000151 & 0.0000007 & 0.0000088 \\
\hline $\begin{array}{l}\text { FEV }_{1} \% \\
\text { predicted } \\
\text { Dyspnea }\end{array}$ & -0.0003460 & 0.0000127 & 0.0000289 & -0.0000006 & 0.0000218 & 0.0001680 & 0.0001090 & -0.0000321 & -0.0000082 & -0.0000563 & -0.0000459 & -0.0000618 & -0.0001240 & -0.0001160 & 0.0000624 & 0.0000002 & 0.0000622 & 0.0000013 & 0.0000333 \\
\hline $\begin{array}{l}\text { (lagged 12 } 12 \\
\text { months), } \\
\text { most days } \\
\text { Dyspnea }\end{array}$ & -0.0330840 & -0.0002340 & 0.0001590 & -0.0000131 & 0.0001680 & 0.0582200 & 0.0482970 & -0.0031610 & -0.0000166 & 0.0000073 & -0.0002790 & -0.0000737 & -0.0011880 & -0.0016420 & -0.0015430 & -0.0000007 & -0.0016600 & -0.0002810 & -0.0000582 \\
\hline $\begin{array}{l}\text { (lagged 12 } \\
\text { months), } \\
\text { several }\end{array}$ & -0.0302390 & -0.0003090 & 0.0003350 & -0.0000102 & 0.0001090 & 0.0482970 & 0.0588700 & -0.0018030 & -0.0000691 & -0.0002550 & -0.0008880 & -0.0003900 & -0.0003880 & -0.0015950 & -0.0030520 & -0.0000017 & -0.0012800 & -0.0001460 & 0.0003330 \\
\hline $\begin{array}{l}\text { Symptom } \\
\text { most days }\end{array}$ & 0.0066220 & -0.0002830 & 0.0006050 & 0.0000026 & -0.0000321 & -0.0031610 & -0.0018030 & 0.0149720 & -0.0000611 & 0.0008050 & -0.0010320 & -0.0029990 & 0.0013300 & 0.0015030 & -0.0002630 & -0.0000031 & 0.0007990 & -0.0000946 & 0.0002410 \\
\hline Age & -0.0070450 & 0.0000188 & -0.0000193 & 0.0000005 & -0.0000082 & -0.0000166 & -0.0000691 & -0.0000611 & 0.0001000 & -0.0000582 & -0.0000547 & 0.0002680 & 0.0001090 & 0.0001400 & 0.0000684 & 0.0000000 & -0.0000763 & 0.0000056 & -0.0001460 \\
\hline $\begin{array}{l}\text { CVD co- } \\
\text { morbidity } \\
\text { (yes) }\end{array}$ & -0.0008520 & 0.0001320 & 0.0004530 & 0.0000025 & -0.0000563 & 0.0000073 & -0.0002550 & 0.0008050 & -0.0000582 & 0.0140810 & -0.0018170 & -0.0000540 & 0.0018910 & -0.0004820 & 0.0006670 & 0.0000006 & 0.0000501 & -0.0000384 & -0.0009500 \\
\hline $\begin{array}{l}\text { Other co- } \\
\text { morbidity } \\
\text { (yes) }\end{array}$ & -0.0075890 & -0.0002220 & -0.0003470 & 0.0000027 & -0.0000459 & -0.0002790 & -0.0008880 & -0.0010320 & -0.0000547 & -0.0018170 & 0.0199030 & 0.0007110 & -0.0026670 & -0.0020980 & 0.0011480 & 0.0000004 & -0.0001750 & -0.0000154 & -0.0002970 \\
\hline $\begin{array}{l}\text { Current } \\
\text { smoker }\end{array}$ & -0.0209270 & 0.0003450 & 0.0000299 & -0.0000025 & -0.0000618 & -0.0000737 & -0.0003900 & -0.0029990 & 0.0002680 & -0.0000540 & 0.0007110 & 0.0158000 & 0.0006350 & 0.0011010 & -0.0017360 & -0.0000008 & 0.0011000 & 0.0000050 & -0.0005780 \\
\hline Female & -0.0064020 & -0.0003680 & 0.0001660 & 0.0000134 & -0.0001240 & -0.0011880 & -0.0003880 & 0.0013300 & 0.0001090 & 0.0018910 & -0.0026670 & 0.0006350 & 0.0172750 & 0.0008540 & -0.0027290 & -0.0000037 & 0.0002310 & 0.0000013 & 0.0006020 \\
\hline $\begin{array}{l}\text { High BMI } \\
\text { category }\end{array}$ & -0.0105250 & 0.0002110 & 0.0006880 & 0.0000131 & -0.0001160 & -0.0016420 & -0.0015950 & 0.0015030 & 0.0001400 & -0.0004820 & -0.0020980 & 0.0011010 & 0.0008540 & 0.0202720 & 0.0050270 & -0.0000049 & -0.0002190 & 0.0000018 & -0.0001300 \\
\hline $\begin{array}{l}\text { Low BMI } \\
\text { category }\end{array}$ & -0.0171820 & 0.0001830 & -0.0005650 & -0.0000033 & 0.0000624 & -0.0015430 & -0.0030520 & -0.0002630 & 0.0000684 & 0.0006670 & 0.0011480 & -0.0017360 & -0.0027290 & 0.0050270 & 0.0237090 & 0.0000106 & 0.0009540 & -0.0000204 & -0.0012160 \\
\hline Fibrinogen & -0.0001370 & -0.0000012 & -0.0000017 & 0.0000000 & 0.0000002 & -0.0000007 & -0.0000017 & -0.0000031 & 0.0000000 & 0.0000006 & 0.0000004 & -0.0000008 & -0.0000037 & -0.0000049 & 0.0000106 & 0.0000002 & -0.0000014 & -0.0000001 & -0.0000049 \\
\hline $\begin{array}{l}\text { Baseline } \\
\text { mMRC }\end{array}$ & -0.0048730 & -0.0002190 & 0.0003520 & 0.0000151 & 0.0000622 & -0.0016600 & -0.0012800 & 0.0007990 & -0.0000763 & 0.0000501 & -0.0001750 & 0.0011000 & 0.0002310 & -0.0002190 & 0.0009540 & -0.0000014 & 0.0214270 & -0.0001930 & -0.0001810 \\
\hline $\begin{array}{l}\text { Baseline } \\
\text { SGRQ }\end{array}$ & -0.0010570 & -0.0000185 & -0.0000380 & 0.0000007 & 0.0000013 & -0.0002810 & -0.0001460 & -0.0000946 & 0.0000056 & -0.0000384 & -0.0000154 & 0.0000050 & 0.0000013 & 0.0000018 & -0.0000204 & -0.0000001 & -0.0001930 & 0.0000169 & -0.0000011 \\
\hline Scale & 0.0148100 & -0.0000354 & 0.0000219 & 0.0000088 & 0.0000333 & -0.0000582 & 0.0003330 & 0.0002410 & -0.0001460 & -0.0009500 & -0.0002970 & -0.0005780 & 0.0006020 & -0.0001300 & -0.0012160 & -0.0000049 & -0.0001810 & -0.0000011 & 0.0028390 \\
\hline
\end{tabular}


Table A18 Covariance matrix (probability of hospitalization)

\begin{tabular}{|c|c|c|c|c|c|c|c|c|c|c|c|c|c|c|c|c|c|c|}
\hline & Intercept & $\begin{array}{l}\text { Time (log } \\
\text { years) }\end{array}$ & $\begin{array}{l}\text { Moderate } \\
\text { exac. count }\end{array}$ & $\begin{array}{c}\text { Severe } \\
\text { exac. count }\end{array}$ & $\begin{array}{l}\mathrm{FEV}_{1} \% \\
\text { predicted }\end{array}$ & $\begin{array}{c}\text { Dyspnea } \\
\text { (most days } \\
\text { vs none) }\end{array}$ & $\begin{array}{l}\text { Dyspnea } \\
\text { (several } \\
\text { days vs } \\
\text { none) }\end{array}$ & $\begin{array}{l}\text { Cough and } \\
\text { sputum }\end{array}$ & Age & $\begin{array}{l}\text { CVD co- } \\
\text { morbidity }\end{array}$ & $\begin{array}{l}\text { Other co- } \\
\text { morbidity }\end{array}$ & Sex & $\begin{array}{l}\text { Current } \\
\text { smoker }\end{array}$ & $\begin{array}{l}\text { BMI (high } \\
\text { vs normal) }\end{array}$ & $\begin{array}{l}\text { BMI (low } \\
\text { vs normal) }\end{array}$ & mMRC & SGRQ & Prior exac. \\
\hline Intercept & 0.2039000 & 0.0002030 & -0.00053300 & 0.0023080 & -0.0004400 & -0.0159600 & -0.0186700 & 0.0009240 & -0.0017600 & 0.0033040 & -0.0025900 & -0.0109800 & -0.0075100 & -0.0015200 & -0.0029600 & -0.0245100 & -0.0003600 & -0.0036800 \\
\hline $\begin{array}{l}\text { Time (log } \\
\text { years) }\end{array}$ & 0.0002030 & 0.0002990 & 0.0000290 & -0.0000600 & -0.0000028 & 0.0004000 & 0.0002300 & 0.0000300 & 0.0000046 & 0.0000028 & -0.0000400 & -0.0000100 & -0.0000600 & 0.0000200 & -0.0000200 & -0.0000800 & -0.0000010 & -0.0000500 \\
\hline $\begin{array}{l}\text { Moderate } \\
\text { exac. count }\end{array}$ & -0.00053300 & 0.0000290 & 0.0002550 & -0.0001100 & 0.0000041 & -0.0002700 & -0.0000900 & -0.0001000 & 0.0000001 & 0.0000400 & -0.0002500 & 0.0000940 & -0.0002100 & -0.0000300 & -0.0001200 & 0.0001930 & 0.0000026 & -0.0000800 \\
\hline $\begin{array}{l}\text { Severe exac. } \\
\text { count }\end{array}$ & 0.0023080 & -0.0000600 & -0.0001100 & 0.0060100 & -0.0000082 & 0.0001470 & -0.0000400 & 0.0000360 & -0.0000500 & -0.0009400 & -0.0002800 & -0.0001900 & -0.0002100 & -0.0010800 & -0.0001100 & -0.0002200 & -0.0000100 & -0.0000500 \\
\hline $\begin{array}{l}\text { FEV }_{1} \% \\
\text { predicted } \\
\text { Dyspnea }\end{array}$ & -0.0004400 & -0.0000028 & 0.0000041 & -0.0000082 & 0.0000093 & 0.0000730 & 0.0000290 & -0.0000200 & -0.0000014 & -0.0000300 & -0.0000200 & -0.0000100 & 0.0000750 & -0.0000200 & 0.0000590 & 0.0000280 & 0.0000010 & 0.0000044 \\
\hline $\begin{array}{l}\text { Dyspnea } \\
\text { (most days } \\
\text { vs none) } \\
\text { Dyspnea }\end{array}$ & -0.0159600 & 0.0004000 & -0.0002700 & 0.0001470 & 0.0000730 & 0.0263900 & 0.0199600 & -0.0020000 & 0.0000019 & -0.0002800 & -0.0009300 & -0.0000900 & -0.0002400 & 0.0004440 & 0.0004650 & -0.0009300 & -0.0000900 & 0.0000150 \\
\hline $\begin{array}{l}\text { (several } \\
\text { days vs } \\
\text { none) }\end{array}$ & -0.0186700 & 0.0002300 & -0.0000900 & -0.0000400 & 0.0000290 & 0.0199600 & 0.0227900 & 0.0001990 & 0.0000170 & 0.0000960 & -0.0002500 & -0.0007000 & -0.0001100 & 0.0001270 & 0.0001760 & -0.0008100 & -0.0000300 & -0.0002000 \\
\hline $\begin{array}{l}\text { Cough and } \\
\text { sputum }\end{array}$ & 0.0009240 & 0.0000300 & -0.0001000 & 0.0000360 & -0.0000200 & -0.0020000 & 0.0001990 & 0.0080500 & 0.0000040 & 0.0005250 & -0.0005300 & -0.0015800 & 0.0004540 & 0.0004970 & -0.0001600 & 0.0000380 & -0.0000400 & -0.0001800 \\
\hline Age & -0.0017600 & 0.0000046 & 0.0000001 & -0.0000500 & -0.0000014 & 0.0000019 & 0.0000170 & 0.0000040 & 0.0000280 & -0.0000500 & -0.0000400 & 0.0001310 & 0.0000430 & 0.0000300 & -0.0000100 & -0.0000700 & 0.0000015 & -0.0000029 \\
\hline $\begin{array}{l}\text { CVD co- } \\
\text { morbidity }\end{array}$ & 0.0033040 & 0.0000028 & 0.0000400 & -0.0009400 & -0.0000300 & -0.0002800 & 0.0000960 & 0.0005250 & -0.0000500 & 0.0106800 & 0.0003050 & -0.0004100 & 0.0003910 & -0.0001000 & -0.0004300 & 0.0002370 & -0.0000200 & 0.0000180 \\
\hline $\begin{array}{l}\text { Other co- } \\
\text { morbidity }\end{array}$ & -0.0025900 & -0.0000400 & -0.0002500 & -0.0002800 & -0.0000200 & -0.0009300 & -0.0002500 & -0.0005300 & -0.0000400 & 0.0003050 & 0.0092630 & 0.0004850 & -0.0011000 & -0.0006600 & 0.0010010 & 0.0000300 & 0.0000076 & 0.0002170 \\
\hline $\begin{array}{l}\text { Current } \\
\text { smoker }\end{array}$ & -0.0109800 & -0.0000100 & 0.0000940 & -0.0001900 & -0.0000100 & -0.0000900 & -0.0007000 & -0.0015800 & 0.0001310 & -0.0004100 & 0.0004850 & 0.0078500 & -0.0004000 & 0.0002180 & -0.0010600 & -0.0000700 & 0.0000130 & -0.0001500 \\
\hline Sex & -0.0075100 & -0.0000600 & -0.0002100 & -0.0002100 & 0.0000750 & -0.0002400 & -0.0001100 & 0.0004540 & 0.0000430 & 0.0003910 & -0.0011000 & -0.0004000 & 0.0106100 & -0.0010200 & 0.0000130 & -0.0000700 & 0.0000076 & 0.0004060 \\
\hline $\begin{array}{l}\text { BMI (high } \\
\text { vs normal) }\end{array}$ & -0.0015200 & 0.0000200 & -0.0000300 & -0.0010800 & -0.0000200 & 0.0004440 & 0.0001270 & 0.0004970 & 0.0000300 & -0.0001000 & -0.0006600 & 0.0002180 & -0.0010200 & 0.0175900 & 0.0024620 & -0.0007200 & -0.0000200 & 0.0002700 \\
\hline $\begin{array}{l}\text { BMI (low vs } \\
\text { normal) }\end{array}$ & -0.0029600 & -0.0000200 & -0.0001200 & -0.0001100 & 0.0000590 & 0.0004650 & 0.0001760 & -0.0001600 & -0.0000100 & -0.0004300 & 0.0010010 & -0.0010600 & 0.0000130 & 0.0024620 & 0.0098460 & -0.0004100 & -0.0000200 & -0.0006300 \\
\hline mMRC & -0.0245100 & -0.0000800 & 0.0001930 & -0.0002200 & 0.0000280 & -0.0009300 & -0.0008100 & 0.0000380 & -0.0000700 & 0.0002370 & 0.0000300 & -0.0000700 & -0.0000700 & -0.0007200 & -0.0004100 & 0.0351500 & -0.0001000 & 0.0004680 \\
\hline SGRQ & -0.0003600 & -0.0000010 & 0.0000026 & -0.0000100 & 0.0000010 & -0.0000900 & -0.0000300 & -0.0000400 & 0.0000015 & -0.0000200 & 0.0000076 & 0.0000130 & 0.0000076 & -0.0000200 & -0.0000200 & -0.0001000 & 0.0000076 & -0.0000400 \\
\hline Prior exac. & -0.0036800 & -0.0000500 & -0.0000800 & -0.0000500 & 0.0000044 & 0.0000150 & -0.0002000 & -0.0001800 & -0.0000029 & 0.0000180 & 0.0002170 & -0.0001500 & 0.0004060 & 0.0002700 & -0.0006300 & 0.0004680 & -0.0000400 & 0.0080900 \\
\hline
\end{tabular}

BMI, body-mass index; CVD, cardiovascular disease; FEV ${ }_{1}$, forced expiratory volume in one second; mMRC, modified Medical Research Council; SGRQ, St George's Respiratory Questionnaire; 6MWD, six-minute walk test distance. 
Table A19 Covariance matrix (ward days)

\begin{tabular}{|c|c|c|c|c|c|c|c|c|c|c|c|c|c|c|c|c|c|c|}
\hline & Intercept & $\begin{array}{c}\text { Time (log } \\
\text { years) }\end{array}$ & $\begin{array}{c}\text { Moderate } \\
\text { exac. count }\end{array}$ & $\begin{array}{c}\text { Severe } \\
\text { exac. count }\end{array}$ & $\begin{array}{l}\text { FEV } 1 \% \\
\text { predicted }\end{array}$ & $\begin{array}{c}\text { Dyspnea } \\
\text { (most days } \\
\text { vs none) }\end{array}$ & $\begin{array}{c}\text { Dyspnea } \\
\text { (several } \\
\text { days vs } \\
\text { none) }\end{array}$ & $\begin{array}{l}\text { Cough and } \\
\text { sputum }\end{array}$ & Age & $\begin{array}{c}\text { CVD co- } \\
\text { morbidity }\end{array}$ & $\begin{array}{l}\text { Other co- } \\
\text { morbidity }\end{array}$ & Sex & $\begin{array}{l}\text { Current } \\
\text { smoker }\end{array}$ & $\begin{array}{l}\text { BMI (high } \\
\text { vs normal) }\end{array}$ & $\begin{array}{c}\text { BMI (low } \\
\text { vs normal) }\end{array}$ & mMRC & SGRQ & Prior exac. \\
\hline Intercept & 0.1091600 & -0.0000820 & 0.0000461 & 0.0002187 & -0.0002020 & -0.0130400 & -0.0145400 & -0.0001030 & -0.0009190 & 0.0012200 & -0.0025030 & -0.0041960 & -0.0035270 & -0.0013090 & -0.0024520 & -0.0109700 & -0.0001730 & -0.0022330 \\
\hline $\begin{array}{l}\text { Time (log } \\
\text { years) }\end{array}$ & -0.0000820 & 0.0001450 & 0.0000150 & 0.0000032 & 0.0000011 & 0.0001536 & 0.0001225 & 0.0000205 & 0.0000010 & -0.0000360 & -0.0000120 & -0.0000310 & 0.0000206 & -0.0000110 & 0.0000445 & 0.0000436 & -0.0000001 & -0.0000110 \\
\hline $\begin{array}{l}\text { Moderate } \\
\text { exac. count }\end{array}$ & 0.0000461 & 0.0000150 & 0.0000646 & -0.0000500 & -0.00000007 & -0.00001290 & -0.0001240 & 0.0000168 & 0.0000005 & 0.0000798 & -0.0000420 & 0.0000386 & -0.0000640 & -0.0000810 & -0.0000140 & -0.0000220 & 0.0000006 & 0.0000250 \\
\hline $\begin{array}{l}\text { Severe exac. } \\
\text { count }\end{array}$ & 0.0002187 & 0.0000032 & -0.0000500 & 0.0001072 & 0.0000045 & 0.0001159 & 0.0001277 & 0.0000593 & -0.0000079 & -0.0000610 & -0.0000430 & -0.0000260 & 0.0001778 & -0.0001140 & -0.0000590 & 0.0000752 & -0.0000023 & -0.0000036 \\
\hline $\begin{array}{l}\mathrm{FEV}_{1} \% \\
\text { predicted }\end{array}$ & -0.0002020 & 0.0000011 & -0.0000007 & 0.0000045 & 0.0000044 & 0.0000304 & 0.0000093 & -0.0000093 & -0.00000007 & -0.0000055 & -0.0000061 & -0.0000100 & 0.0000374 & -0.00000170 & 0.0000283 & 0.0000088 & 0.0000005 & 0.0000068 \\
\hline $\begin{array}{l}\text { Dyspnea } \\
\text { (most days } \\
\text { vs none) }\end{array}$ & -0.0130400 & 0.0001536 & -0.0001290 & 0.0001159 & 0.0000304 & 0.0145100 & 0.0120700 & -0.0013250 & 0.0000259 & 0.0003144 & 0.0000296 & -0.0003540 & -0.0000720 & -0.0001480 & 0.0010140 & -0.0001980 & -0.0000280 & 0.0001809 \\
\hline $\begin{array}{l}\text { Dyspnea } \\
\text { (several } \\
\text { days vs } \\
\text { none) }\end{array}$ & -0.0145400 & 0.0001225 & -0.0001240 & 0.0001277 & 0.0000093 & 0.0120700 & 0.0136900 & -0.0002080 & 0.0000368 & 0.0003118 & 0.0001811 & -0.0003560 & 0.0000320 & 0.0003139 & 0.0007298 & -0.0000550 & -0.0000019 & -0.0001144 \\
\hline $\begin{array}{l}\text { Cough and } \\
\text { sputum }\end{array}$ & -0.0001030 & 0.0000205 & 0.0000168 & 0.0000593 & -0.0000093 & -0.0013250 & -0.0002080 & 0.0037650 & 0.0000025 & -0.0000470 & -0.0001200 & -0.0005950 & 0.0001126 & 0.0005259 & -0.0001040 & 0.0004401 & -0.0000180 & -0.0001050 \\
\hline Age & -0.0009190 & 0.0000010 & 0.0000005 & -0.0000079 & -0.0000000 & 0.0000259 & 0.0000368 & 0.0000025 & 0.0000140 & -0.0000300 & -0.0000130 & 0.0000539 & 0.0000165 & 0.0000246 & -0.0000005 & -0.0000340 & 0.0000005 & 0.0000018 \\
\hline $\begin{array}{l}\text { CVD co- } \\
\text { morbidity }\end{array}$ & 0.0012200 & -0.0000360 & 0.0000798 & -0.0000610 & -0.0000055 & 0.0003144 & 0.0003118 & -0.0000470 & -0.0000300 & 0.0041730 & 0.0002184 & -0.0000410 & -0.0000030 & 0.0000479 & 0.0000171 & -0.0001600 & -0.0000099 & 0.0000765 \\
\hline $\begin{array}{l}\text { Other co- } \\
\text { morbidity }\end{array}$ & -0.0025030 & -0.0000120 & -0.0000420 & -0.0000430 & -0.0000061 & 0.0000296 & 0.0001811 & -0.0001200 & -0.0000130 & 0.0002184 & 0.0047760 & 0.0004087 & -0.0006140 & -0.0001980 & 0.0002566 & 0.0001125 & -0.0000048 & 0.0000929 \\
\hline $\begin{array}{l}\text { Current } \\
\text { smoker }\end{array}$ & -0.0041960 & -0.0000310 & 0.0000386 & -0.0000260 & -0.0000100 & -0.0003540 & -0.0003560 & -0.0005950 & 0.0000539 & -0.0000410 & 0.0004087 & 0.0036990 & -0.0004520 & 0.0000613 & -0.0003120 & -0.0000590 & -0.0000009 & 0.0000977 \\
\hline Sex & -0.0035270 & 0.0000206 & -0.0000640 & 0.0001778 & 0.0000374 & -0.0000720 & 0.0000320 & 0.0001126 & 0.0000165 & -0.0000030 & -0.0006140 & -0.0004520 & 0.0055080 & -0.0003620 & -0.0000440 & 0.0000696 & 0.0000041 & -0.0000350 \\
\hline $\begin{array}{l}\text { BMI (high } \\
\text { vs normal) }\end{array}$ & -0.0013090 & -0.0000110 & -0.0000810 & -0.0001140 & -0.0000170 & -0.0001480 & 0.0003139 & 0.0005259 & 0.0000246 & 0.0000479 & -0.0001980 & 0.0000613 & -0.0003620 & 0.0081920 & 0.0014770 & -0.0006580 & -0.0000120 & 0.0007783 \\
\hline $\begin{array}{l}\text { BMI (low vs } \\
\text { normal) }\end{array}$ & -0.0024520 & 0.0000445 & -0.0000140 & -0.0000590 & 0.0000283 & 0.0010140 & 0.0007298 & -0.0001040 & -0.00000005 & 0.0000171 & 0.0002566 & -0.0003120 & -0.0000440 & 0.0014770 & 0.0042600 & -0.0003420 & -0.0000051 & -0.0002560 \\
\hline mMRC & -0.0109700 & 0.0000436 & -0.0000220 & 0.0000752 & 0.0000088 & -0.0001980 & -0.0000550 & 0.0004401 & -0.0000340 & -0.0001600 & 0.0001125 & -0.0000590 & 0.0000696 & -0.0006580 & -0.0003420 & 0.0163000 & -0.0000440 & -0.0001750 \\
\hline SGRQ & -0.0001730 & -0.0000001 & 0.0000006 & -0.0000023 & 0.0000005 & -0.0000280 & -0.0000019 & -0.0000180 & 0.0000005 & -0.0000099 & -0.0000048 & -0.0000009 & 0.0000041 & -0.0000120 & -0.0000051 & -0.0000440 & 0.0000037 & -0.0000180 \\
\hline Prior exac. & -0.0022330 & -0.0000110 & 0.0000250 & -0.0000036 & 0.0000068 & 0.0001809 & -0.0001140 & -0.0001050 & 0.0000018 & 0.0000765 & 0.0000929 & 0.0000977 & -0.0000350 & 0.0007783 & -0.0002560 & -0.0001750 & -0.0000180 & 0.0039300 \\
\hline
\end{tabular}


Table A20 Covariance matrix (intensive care unit)

\begin{tabular}{|c|c|c|c|c|c|c|c|c|c|c|c|c|c|c|c|c|c|c|}
\hline & Intercept & $\begin{array}{c}\text { Time (log } \\
\text { years) }\end{array}$ & $\begin{array}{c}\text { Moderate } \\
\text { exac. count }\end{array}$ & $\begin{array}{c}\text { Severe } \\
\text { exac. count }\end{array}$ & $\begin{array}{l}\mathrm{FEV}_{1} \% \\
\text { predicted }\end{array}$ & $\begin{array}{c}\text { Dyspnea } \\
\text { (most days } \\
\text { vs none) }\end{array}$ & $\begin{array}{l}\text { Dyspnea } \\
\text { (several } \\
\text { days vs } \\
\text { none) }\end{array}$ & $\begin{array}{c}\text { Cough and } \\
\text { sputum }\end{array}$ & Age & $\begin{array}{c}\text { CVD co- } \\
\text { morbidity }\end{array}$ & $\begin{array}{l}\text { Other co- } \\
\text { morbidity }\end{array}$ & Sex & $\begin{array}{l}\text { Current } \\
\text { smoker }\end{array}$ & $\begin{array}{l}\text { BMI (high } \\
\text { vs normal) }\end{array}$ & $\begin{array}{l}\text { BMI (low } \\
\text { vs normal) }\end{array}$ & mMRC & SGRQ & Prior exac. \\
\hline Intercept & 1.1393100 & -0.0016900 & 0.0012370 & 0.0034540 & -0.0023400 & -0.1097300 & -0.1224800 & -0.0000670 & -0.0106000 & 0.0081730 & -0.0461400 & -0.0618700 & -0.0387200 & -0.0248300 & -0.0275800 & -0.0460100 & -0.0020340 & -0.0157500 \\
\hline $\begin{array}{l}\text { Time (log } \\
\text { years) }\end{array}$ & -0.0016900 & 0.0020300 & 0.0001408 & 0.0001762 & 0.0000177 & 0.0015040 & 0.0013500 & 0.0001955 & 0.0000196 & -0.0005020 & -0.0003470 & -0.0004870 & 0.0000077 & 0.0001811 & 0.0004686 & 0.0001856 & 0.0000001 & -0.0001770 \\
\hline $\begin{array}{l}\text { Moderate } \\
\text { exac. count }\end{array}$ & 0.0012370 & 0.0001408 & 0.0004546 & -0.0004010 & -0.0000120 & -0.0011830 & -0.00008580 & -0.0001970 & -0.0000110 & 0.0008472 & -0.0004340 & 0.0004293 & -0.0010230 & -0.0003530 & -0.0001970 & -0.0001440 & 0.0000075 & 0.0000856 \\
\hline $\begin{array}{l}\text { Severe exac. } \\
\text { count }\end{array}$ & 0.0034540 & 0.0001762 & -0.0004010 & 0.0010000 & 0.0000509 & 0.0002784 & 0.0008151 & 0.0012930 & -0.0000870 & -0.0002570 & -0.0002940 & -0.0001340 & 0.0020560 & -0.0017940 & -0.0005790 & 0.0003371 & -0.0000290 & 0.0002909 \\
\hline $\begin{array}{l}\mathrm{FEV}_{1} \% \\
\text { predicted }\end{array}$ & -0.0023400 & 0.0000177 & -0.0000120 & 0.0000509 & 0.0000514 & 0.0004166 & 0.0001538 & -0.0001040 & -0.0000087 & -0.0000410 & -0.0000660 & -0.0000880 & 0.0003959 & -0.0001080 & 0.0003339 & 0.0001027 & 0.0000061 & 0.0000707 \\
\hline $\begin{array}{l}\text { Dyspnea } \\
\text { (most days } \\
\text { vs none) } \\
\text { Dyspnea }\end{array}$ & -0.1097300 & 0.0015040 & -0.0011830 & 0.0002784 & 0.0004166 & 0.1292900 & 0.0936600 & -0.0145100 & 0.0003501 & 0.0008511 & -0.0007060 & -0.0034860 & -0.0000880 & -0.0005230 & 0.0133400 & -0.0030620 & -0.0003430 & 0.0040200 \\
\hline $\begin{array}{l}\text { (several } \\
\text { days vs } \\
\text { none) }\end{array}$ & -0.1224800 & 0.0013500 & -0.0008580 & 0.0008151 & 0.0001538 & 0.0936600 & 0.1092300 & -0.0009320 & 0.0003995 & 0.0017940 & 0.0014470 & -0.0028600 & 0.0000523 & 0.0052300 & 0.0076350 & -0.0009980 & -0.0000260 & -0.0005070 \\
\hline $\begin{array}{l}\text { Cough and } \\
\text { sputum }\end{array}$ & -0.0000670 & 0.0001955 & -0.0001970 & 0.0012930 & -0.0001040 & -0.0145100 & -0.0009320 & 0.0453800 & 0.0000243 & -0.0004220 & -0.0020870 & -0.0067460 & 0.0010150 & 0.0076630 & -0.0007750 & 0.0045430 & -0.0002170 & -0.0013030 \\
\hline Age & -0.0106000 & 0.0000196 & -0.0000110 & -0.0000870 & -0.0000087 & 0.0003501 & 0.0003995 & 0.0000243 & 0.0001619 & -0.0002940 & -0.0001880 & 0.0007178 & 0.0002506 & 0.0003265 & -0.0000086 & -0.0002500 & 0.0000058 & -0.0000277 \\
\hline $\begin{array}{l}\text { CVD co- } \\
\text { morbidity }\end{array}$ & 0.0081730 & -0.0005020 & 0.0008472 & -0.0002570 & -0.0000410 & 0.0008511 & 0.0017940 & -0.0004220 & -0.0002940 & 0.0404600 & -0.0007180 & -0.0009620 & -0.0004050 & -0.0005300 & -0.0009670 & -0.0008500 & -0.0000850 & 0.0014820 \\
\hline $\begin{array}{l}\text { Other co- } \\
\text { morbidity }\end{array}$ & -0.0461400 & -0.0003470 & -0.0004340 & -0.0002940 & -0.0000660 & -0.0007060 & 0.0014470 & -0.0020870 & -0.0001880 & -0.0007180 & 0.0743400 & 0.0033810 & -0.0063760 & -0.0034010 & 0.0018260 & 0.0012870 & -0.0000220 & 0.0007403 \\
\hline $\begin{array}{l}\text { Current } \\
\text { smoker }\end{array}$ & -0.0618700 & -0.0004870 & 0.0004293 & -0.0001340 & -0.0000880 & -0.0034860 & -0.0028600 & -0.0067460 & 0.0007178 & -0.0009620 & 0.0033810 & 0.0421300 & -0.0038860 & -0.0015410 & -0.0038170 & -0.0004660 & 0.0000250 & 0.0005934 \\
\hline Sex & -0.0387200 & 0.0000077 & -0.0010230 & 0.0020560 & 0.0003959 & -0.0000880 & 0.0000523 & 0.0010150 & 0.0002506 & -0.0004050 & -0.0063760 & -0.0038860 & 0.0922400 & -0.0024060 & -0.0008090 & -0.0006120 & 0.0000463 & -0.0014900 \\
\hline $\begin{array}{l}\text { BMI (high } \\
\text { vs normal) }\end{array}$ & -0.0248300 & 0.0001811 & -0.0003530 & -0.0017940 & -0.0001080 & -0.0005230 & 0.0052300 & 0.0076630 & 0.0003265 & -0.0005300 & -0.0034010 & -0.0015410 & -0.0024060 & 0.0726300 & 0.0202100 & -0.0055540 & -0.0001460 & 0.0092990 \\
\hline $\begin{array}{l}\text { BMI (low vs } \\
\text { normal) }\end{array}$ & -0.0275800 & 0.0004686 & -0.0001970 & -0.0005790 & 0.0003339 & 0.0133400 & 0.0076350 & -0.0007750 & -0.0000086 & -0.0009670 & 0.0018260 & -0.0038170 & -0.0008090 & 0.0202100 & 0.0549000 & -0.0022130 & -0.0001060 & -0.0040450 \\
\hline mMRC & -0.0460100 & 0.0001856 & -0.0001440 & 0.0003371 & 0.0001027 & -0.0030620 & -0.0009980 & 0.0045430 & -0.0002500 & -0.0008500 & 0.0012870 & -0.0004660 & -0.0006120 & -0.0055540 & -0.0022130 & 0.0994600 & -0.0005240 & -0.0015840 \\
\hline SGRQ & -0.0020340 & 0.0000001 & 0.0000075 & -0.0000290 & 0.0000061 & -0.0003430 & -0.0000260 & -0.0002170 & 0.0000058 & -0.0000850 & -0.0000220 & 0.0000250 & 0.0000463 & -0.0001460 & -0.0001060 & -0.0005240 & 0.0000434 & -0.0002010 \\
\hline Prior exac. & -0.0157500 & -0.0001770 & 0.0000856 & 0.0002909 & 0.0000707 & 0.0040200 & -0.0005070 & -0.0013030 & -0.0000277 & 0.0014820 & 0.0007403 & 0.0005934 & -0.0014900 & 0.0092990 & -0.0040450 & -0.0015840 & -0.0002010 & 0.0394000 \\
\hline
\end{tabular}

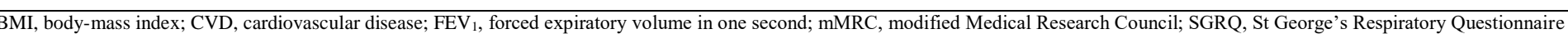


Table A21 Covariance matrix (emergency room visits)

\begin{tabular}{|c|c|c|c|c|c|c|c|c|c|c|c|c|c|c|c|c|c|c|}
\hline & Intercept & $\begin{array}{c}\text { Time (log } \\
\text { years) }\end{array}$ & $\begin{array}{c}\text { Moderate } \\
\text { exac. count }\end{array}$ & $\begin{array}{c}\text { Severe } \\
\text { exac. count }\end{array}$ & $\begin{array}{l}\mathbf{F E V}_{1} \% \\
\text { predicted }\end{array}$ & $\begin{array}{c}\text { Dyspnea } \\
\text { (most days } \\
\text { vs none) }\end{array}$ & $\begin{array}{c}\text { Dyspnea } \\
\text { (several } \\
\text { days vs } \\
\text { none) }\end{array}$ & $\begin{array}{l}\text { Cough and } \\
\text { sputum }\end{array}$ & Age & $\begin{array}{c}\text { CVD co- } \\
\text { morbidity }\end{array}$ & $\begin{array}{l}\text { Other co- } \\
\text { morbidity }\end{array}$ & Sex & $\begin{array}{l}\text { Current } \\
\text { smoker }\end{array}$ & $\begin{array}{l}\text { BMI (high } \\
\text { vs normal) }\end{array}$ & $\begin{array}{l}\text { BMI (low } \\
\text { vs normal) }\end{array}$ & mMRC & SGRQ & Prior exac. \\
\hline Intercept & 0.8437400 & 0.0005724 & 0.0000682 & 0.0032500 & -0.0017690 & -0.0885400 & -0.1026900 & 0.0031460 & -0.0077180 & 0.0086530 & -0.0149000 & -0.0375100 & -0.0301700 & -0.0083870 & -0.0188900 & -0.0500700 & -0.0013680 & -0.0165400 \\
\hline $\begin{array}{l}\text { Time (log } \\
\text { years) }\end{array}$ & 0.0005724 & 0.0010800 & 0.0000125 & 0.0000641 & 0.0000018 & 0.0010690 & 0.0006949 & 0.0000284 & 0.0000087 & -0.0000710 & -0.0000640 & -0.0001980 & -0.0000140 & 0.0000760 & 0.0001486 & -0.0000460 & 0.0000024 & -0.0000390 \\
\hline $\begin{array}{l}\text { Moderate } \\
\text { exac. count }\end{array}$ & 0.0000682 & 0.0000125 & 0.0002859 & -0.0003060 & -0.0000032 & -0.0005830 & -0.0003290 & -0.0001410 & -0.0000003 & 0.0001140 & -0.0005290 & 0.0001047 & -0.0006240 & -0.0002270 & -0.0000920 & 0.0001730 & 0.0000022 & -0.0000590 \\
\hline $\begin{array}{l}\text { Severe exac. } \\
\text { count }\end{array}$ & 0.0032500 & 0.0000641 & -0.0003060 & 0.0010920 & 0.0000253 & 0.0002766 & 0.0000574 & 0.0006597 & -0.0000570 & 0.0002649 & -0.0000190 & 0.0000576 & 0.0013730 & -0.0015890 & -0.0005120 & -0.0002840 & -0.0000150 & 0.0001079 \\
\hline $\begin{array}{l}\mathrm{FEV}_{\mathbf{1}} \% \\
\text { predicted }\end{array}$ & -0.0017690 & 0.0000018 & -0.0000032 & 0.0000253 & 0.0000364 & 0.0002578 & 0.0001145 & -0.0000740 & -0.0000053 & -0.0000530 & -0.0000320 & -0.0000870 & 0.0002893 & -0.0001200 & 0.0002316 & 0.0000600 & 0.0000052 & 0.0000507 \\
\hline $\begin{array}{l}\text { Dyspot } \\
\text { (most days } \\
\text { vs none) } \\
\text { Dryspre) }\end{array}$ & -0.0885400 & 0.0010690 & -0.0005830 & 0.0002766 & 0.0002578 & 0.1255400 & 0.1028400 & -0.0090920 & 0.0000150 & -0.0004160 & -0.0024440 & -0.0008110 & -0.0013060 & -0.0000480 & 0.0047950 & -0.0025640 & -0.0003520 & -0.0003250 \\
\hline $\begin{array}{l}\text { (several } \\
\text { days vs } \\
\text { none) }\end{array}$ & -0.1026900 & 0.0006949 & -0.0003290 & 0.0000574 & 0.0001145 & 0.1028400 & 0.1134000 & -0.0010350 & 0.0001238 & -0.0005190 & -0.0005920 & -0.0018950 & -0.0006020 & 0.0020650 & 0.0029690 & -0.0009330 & -0.0001120 & -0.0019200 \\
\hline $\begin{array}{l}\text { Cough and } \\
\text { sputum }\end{array}$ & 0.0031460 & 0.0000284 & -0.0001410 & 0.0006597 & -0.0000740 & -0.0090920 & -0.0010350 & 0.0302700 & -0.0000046 & -0.0003810 & -0.0012290 & -0.0055440 & 0.0010100 & 0.0022750 & -0.0010140 & 0.0017620 & -0.0001320 & -0.0004810 \\
\hline Age & -0.0077180 & 0.0000087 & -0.0000003 & -0.0000570 & -0.0000053 & 0.0000150 & 0.0001238 & -0.0000046 & 0.0001171 & -0.0002100 & -0.0001830 & 0.0004841 & 0.0001939 & 0.0001421 & -0.0000130 & -0.0001410 & 0.0000047 & 0.0000409 \\
\hline $\begin{array}{l}\text { CVD co- } \\
\text { morbidity }\end{array}$ & 0.0086530 & -0.0000710 & 0.0001140 & 0.0002649 & -0.0000530 & -0.0004160 & -0.0005190 & -0.0003810 & -0.0002100 & 0.0380100 & 0.0012460 & 0.0008099 & -0.0004420 & 0.0011930 & -0.0000640 & -0.0000470 & -0.0000510 & 0.0006794 \\
\hline $\begin{array}{l}\text { Other co- } \\
\text { morbidity }\end{array}$ & -0.0149000 & -0.0000640 & -0.0005290 & -0.0000190 & -0.0000320 & -0.0024440 & -0.0005920 & -0.0012290 & -0.0001830 & 0.0012460 & 0.0404100 & 0.0022980 & -0.0043620 & -0.0014670 & 0.0027620 & 0.0008016 & -0.0000230 & 0.0010860 \\
\hline $\begin{array}{l}\text { Current } \\
\text { smoker }\end{array}$ & -0.0375100 & -0.0001980 & 0.0001047 & 0.0000576 & -0.0000870 & -0.0008110 & -0.0018950 & -0.0055440 & 0.0004841 & 0.0008099 & 0.0022980 & 0.0310200 & -0.0024680 & 0.0014060 & -0.0034280 & 0.0000900 & -0.0000160 & 0.0001707 \\
\hline Sex & -0.0301700 & -0.0000140 & -0.0006240 & 0.0013730 & 0.0002893 & -0.0013060 & -0.0006020 & 0.0010100 & 0.0001939 & -0.0004420 & -0.0043620 & -0.0024680 & 0.0430100 & -0.0025490 & -0.0005650 & -0.0009060 & 0.0000561 & 0.0000519 \\
\hline $\begin{array}{l}\text { BMI (high } \\
\text { vs normal) }\end{array}$ & -0.0083870 & 0.0000760 & -0.0002270 & -0.0015890 & -0.0001200 & -0.0000480 & 0.0020650 & 0.0022750 & 0.0001421 & 0.0011930 & -0.0014670 & 0.0014060 & -0.0025490 & 0.0776600 & 0.0111700 & -0.0032020 & -0.0000940 & 0.0045200 \\
\hline $\begin{array}{l}\text { BMI (low vs } \\
\text { normal) }\end{array}$ & -0.0188900 & 0.0001486 & -0.0000920 & -0.0005120 & 0.0002316 & 0.0047950 & 0.0029690 & -0.0010140 & -0.0000130 & -0.0000640 & 0.0027620 & -0.0034280 & -0.0005650 & 0.0111700 & 0.0346400 & -0.0004850 & -0.0000450 & -0.0019800 \\
\hline mMRC & -0.0500700 & -0.0000460 & 0.0001730 & -0.0002840 & 0.0000600 & -0.0025640 & -0.0009330 & 0.0017620 & -0.0001410 & -0.0000470 & 0.0008016 & 0.0000900 & -0.0009060 & -0.0032020 & -0.0004850 & 0.0805600 & -0.0003020 & -0.0004560 \\
\hline SGRQ & -0.0013680 & 0.0000024 & 0.0000022 & -0.0000150 & 0.0000052 & -0.0003520 & -0.0001120 & -0.0001320 & 0.0000047 & -0.0000510 & -0.0000230 & -0.0000160 & 0.0000561 & -0.0000940 & -0.0000450 & -0.0003020 & 0.0000294 & -0.0001150 \\
\hline Prior exac. & -0.0165400 & -0.0000390 & -0.0000590 & 0.0001079 & 0.0000507 & -0.0003250 & -0.0019200 & -0.0004810 & 0.0000409 & 0.0006794 & 0.0010860 & 0.0001707 & 0.0000519 & 0.0045200 & -0.0019800 & -0.0004560 & -0.0001150 & 0.0285600 \\
\hline
\end{tabular}


Table A22 Covariance matrix (days and nights at home)

\begin{tabular}{|c|c|c|c|c|c|c|c|c|c|c|c|c|c|c|c|c|c|c|}
\hline & Intercept & $\begin{array}{c}\text { Time (log } \\
\text { years) }\end{array}$ & $\begin{array}{c}\text { Moderate } \\
\text { exac. count }\end{array}$ & $\begin{array}{c}\text { Severe } \\
\text { exac. count }\end{array}$ & $\begin{array}{l}\text { FEV } 1 \% \\
\text { predicted }\end{array}$ & $\begin{array}{c}\text { Dyspnea } \\
\text { (most days } \\
\text { vs none) }\end{array}$ & $\begin{array}{c}\text { Dyspnea } \\
\text { (several } \\
\text { days vs } \\
\text { none) }\end{array}$ & $\begin{array}{l}\text { Cough and } \\
\text { sputum }\end{array}$ & Age & $\begin{array}{c}\text { CVD co- } \\
\text { morbidity }\end{array}$ & $\begin{array}{l}\text { Other co- } \\
\text { morbidity }\end{array}$ & Sex & $\begin{array}{l}\text { Current } \\
\text { smoker }\end{array}$ & $\begin{array}{l}\text { BMI (high } \\
\text { vs normal) }\end{array}$ & $\begin{array}{c}\text { BMI (low } \\
\text { vs normal) }\end{array}$ & mMRC & SGRQ & Prior exac. \\
\hline Intercept & 2.8069600 & 0.0042910 & -0.0028370 & 0.0104500 & -0.0045420 & -0.1197100 & -0.1612000 & 0.0111200 & -0.0213600 & 0.0202900 & -0.0708200 & -0.1012200 & -0.0938700 & -0.0231900 & -0.0413300 & -0.6375400 & -0.0034670 & -0.0985200 \\
\hline $\begin{array}{l}\text { Time (log } \\
\text { years) }\end{array}$ & 0.0042910 & 0.0029070 & 0.0001068 & -0.00008880 & -0.0000040 & 0.0034790 & 0.0021130 & 0.0006597 & 0.0000178 & -0.0000870 & -0.0001640 & -0.0005230 & -0.0000770 & -0.0004100 & 0.0001206 & 0.0000132 & -0.0000160 & 0.0001079 \\
\hline $\begin{array}{l}\text { Moderate } \\
\text { exac. count }\end{array}$ & -0.0028370 & 0.0001068 & 0.0012540 & -0.0009310 & -0.0000020 & -0.0018440 & -0.0009680 & -0.0004530 & 0.0000265 & 0.0003873 & -0.0014790 & 0.0004079 & -0.0018130 & -0.0007520 & -0.0005650 & 0.0009193 & 0.0000093 & -0.0001980 \\
\hline $\begin{array}{l}\text { Severe exac. } \\
\text { count }\end{array}$ & 0.0104500 & -0.0000880 & -0.0009310 & 0.0062780 & 0.0000874 & 0.0021000 & 0.0009182 & 0.0010620 & -0.0001910 & -0.0005110 & -0.0006910 & 0.0001064 & 0.0031080 & -0.0006130 & -0.0019520 & -0.0006310 & -0.0000700 & -0.0006130 \\
\hline $\begin{array}{l}\mathrm{FEV}_{1} \% \\
\text { predicted }\end{array}$ & -0.0045420 & -0.0000040 & -0.0000020 & 0.0000874 & 0.0000883 & 0.0005654 & 0.0002347 & -0.0001470 & -0.0000120 & -0.0001244 & -0.0000810 & -0.0002000 & 0.0008312 & -0.0003740 & 0.0005766 & 0.0002149 & 0.0000112 & 0.0001635 \\
\hline $\begin{array}{l}\text { Dyspnea } \\
\text { (most days } \\
\text { vs none) }\end{array}$ & -0.1197100 & 0.0034790 & -0.0018440 & 0.0021000 & 0.0005654 & 0.2275900 & 0.1607700 & -0.0198800 & 0.0000956 & -0.0033810 & -0.0050760 & -0.0042420 & -0.0041450 & 0.0004823 & 0.0101700 & -0.0061720 & -0.0008820 & -0.0002390 \\
\hline $\begin{array}{l}\text { Dyspnea } \\
\text { (several } \\
\text { days vs } \\
\text { none) }\end{array}$ & -0.1612000 & 0.0021130 & -0.0009680 & 0.0009182 & 0.0002347 & 0.1607700 & 0.1853400 & -0.0019470 & 0.0003588 & -0.0023290 & -0.0008440 & -0.0051190 & -0.0017550 & 0.0040100 & 0.0081660 & -0.0022910 & -0.0002770 & -0.0034500 \\
\hline $\begin{array}{l}\text { Cough and } \\
\text { sputum }\end{array}$ & 0.0111200 & 0.0006597 & -0.0004530 & 0.0010620 & -0.0001470 & -0.0198800 & -0.0019470 & 0.0780700 & -0.0000500 & -0.0001810 & -0.0022340 & -0.0141300 & 0.0016400 & 0.0029740 & -0.0012240 & 0.0031790 & -0.0003850 & -0.0001800 \\
\hline Age & -0.0213600 & 0.0000178 & 0.0000265 & -0.0001910 & -0.0000120 & 0.0000956 & 0.0003588 & -0.0000500 & 0.0003168 & -0.0005410 & -0.0004640 & 0.0012480 & 0.0006211 & 0.0004760 & -0.0001470 & -0.0003010 & 0.0000127 & 0.0000845 \\
\hline $\begin{array}{l}\text { CVD co- } \\
\text { morbidity }\end{array}$ & 0.0202900 & -0.0000870 & 0.0003873 & -0.0005110 & -0.0001240 & -0.0033810 & -0.0023290 & -0.0001810 & -0.0005410 & 0.0876800 & 0.0044070 & 0.0009843 & -0.0009580 & 0.0017830 & 0.0000637 & 0.0021200 & -0.0001340 & 0.0019790 \\
\hline $\begin{array}{l}\text { Other co- } \\
\text { morbidity }\end{array}$ & -0.0708200 & -0.0001640 & -0.0014790 & -0.0006910 & -0.0000810 & -0.0050760 & -0.00008440 & -0.0022340 & -0.0004640 & 0.0044070 & 0.1315000 & 0.0049580 & -0.0108300 & -0.0052270 & 0.0097800 & 0.0030250 & -0.0001040 & 0.0029260 \\
\hline $\begin{array}{l}\text { Current } \\
\text { smoker }\end{array}$ & -0.1012200 & -0.0005230 & 0.0004079 & 0.0001064 & -0.0002000 & -0.0042420 & -0.0051190 & -0.0141300 & 0.0012480 & 0.0009843 & 0.0049580 & 0.0805600 & -0.0070460 & 0.0044830 & -0.0106000 & 0.0028260 & 0.0000111 & 0.0002523 \\
\hline Sex & -0.0938700 & -0.0000770 & -0.0018130 & 0.0031080 & 0.0008312 & -0.0041450 & -0.0017550 & 0.0016400 & 0.0006211 & -0.0009580 & -0.0108300 & -0.0070460 & 0.1096000 & -0.0084730 & -0.0001850 & -0.0012200 & 0.0001424 & 0.0014420 \\
\hline $\begin{array}{l}\text { BMI (high } \\
\text { vs normal) }\end{array}$ & -0.0231900 & -0.0004100 & -0.0007520 & -0.0006130 & -0.0003740 & 0.0004823 & 0.0040100 & 0.0029740 & 0.0004760 & 0.0017830 & -0.0052270 & 0.0044830 & -0.0084730 & 0.2353300 & 0.0237900 & -0.0106200 & -0.0001640 & 0.0049220 \\
\hline $\begin{array}{l}\text { BMI (low vs } \\
\text { normal) }\end{array}$ & -0.0413300 & 0.0001206 & -0.0005650 & -0.0019520 & 0.0005766 & 0.0101700 & 0.0081660 & -0.0012240 & -0.0001470 & 0.0000637 & 0.0097800 & -0.0106000 & -0.0001850 & 0.0237900 & 0.0933600 & -0.0013580 & -0.0001330 & -0.0025490 \\
\hline mMRC & -0.6375400 & 0.0000132 & 0.0009193 & -0.0006310 & 0.0002149 & -0.0061720 & -0.0022910 & 0.0031790 & -0.0003010 & 0.0021200 & 0.0030250 & 0.0028260 & -0.0012200 & -0.0106200 & -0.0013580 & 0.7060700 & -0.0008210 & 0.0001734 \\
\hline SGRQ & -0.0034670 & -0.0000160 & 0.0000093 & -0.0000700 & 0.0000112 & -0.0008820 & -0.0002770 & -0.0003850 & 0.0000127 & -0.0001340 & -0.0001040 & 0.0000111 & 0.0001424 & -0.0001640 & -0.0001330 & -0.0008210 & 0.0000739 & -0.0003290 \\
\hline Prior exac. & -0.0985200 & 0.0001079 & -0.0001980 & -0.0006130 & 0.0001635 & -0.0002390 & -0.0034500 & -0.0001800 & 0.0000845 & 0.0019790 & 0.0029260 & 0.0002523 & 0.0014420 & 0.0049220 & -0.0025490 & 0.0001734 & -0.0003290 & 0.1233000 \\
\hline
\end{tabular}


Table A23 Covariance matrix (office visits)

\begin{tabular}{|c|c|c|c|c|c|c|c|c|c|c|c|c|c|c|c|c|c|c|}
\hline & Intercept & $\begin{array}{c}\text { Time (log } \\
\text { years) }\end{array}$ & $\begin{array}{c}\text { Moderate } \\
\text { exac. count }\end{array}$ & $\begin{array}{c}\text { Severe } \\
\text { exac. count }\end{array}$ & $\begin{array}{l}\mathrm{FEV}_{1} \% \\
\text { predicted }\end{array}$ & $\begin{array}{c}\text { Dyspnea } \\
\text { (most days } \\
\text { vs none) }\end{array}$ & $\begin{array}{l}\text { Dyspnea } \\
\text { (several } \\
\text { days vs } \\
\text { none) }\end{array}$ & $\begin{array}{c}\text { Cough and } \\
\text { sputum }\end{array}$ & Age & $\begin{array}{c}\text { CVD co- } \\
\text { morbidity }\end{array}$ & $\begin{array}{l}\text { Other co- } \\
\text { morbidity }\end{array}$ & Sex & $\begin{array}{l}\text { Current } \\
\text { smoker }\end{array}$ & $\begin{array}{l}\text { BMI (high } \\
\text { vs normal) }\end{array}$ & $\begin{array}{l}\text { BMI (low } \\
\text { vs normal) }\end{array}$ & mMRC & SGRQ & Prior exac. \\
\hline Intercept & 0.1587500 & 0.0001214 & 0.0000996 & 0.0006582 & -0.0003490 & -0.0258700 & -0.0284300 & 0.0008488 & -0.0012750 & 0.0008523 & -0.0049280 & -0.0068270 & -0.0058710 & -0.0008890 & -0.0035210 & -0.0122200 & -0.0002420 & -0.0031080 \\
\hline $\begin{array}{l}\text { Time (log } \\
\text { years) }\end{array}$ & 0.0001214 & 0.0002103 & 0.0000052 & -0.0000360 & 0.0000000 & 0.0002090 & 0.0001219 & 0.0000162 & 0.0000011 & 0.0000050 & -0.0000030 & -0.0000240 & -0.0000110 & -0.0000200 & 0.0000070 & 0.0000035 & 0.0000002 & 0.0000018 \\
\hline $\begin{array}{l}\text { Moderate } \\
\text { exac. count }\end{array}$ & 0.0000996 & 0.0000052 & 0.0000403 & -0.00005990 & -0.0000000 & -0.0000600 & -0.0000330 & -0.0000140 & -0.0000010 & 0.0000020 & -0.0000960 & 0.0000161 & -0.0001100 & -0.0000480 & -0.0000110 & 0.0000269 & -0.0000004 & -0.0000220 \\
\hline $\begin{array}{l}\text { Severe exac. } \\
\text { count }\end{array}$ & 0.0006582 & -0.0000360 & -0.0000590 & 0.0007045 & 0.0000043 & -0.0000680 & -0.0000300 & -0.0000140 & -0.0000130 & 0.0000004 & -0.0000210 & -0.0000250 & 0.0002469 & -0.0000260 & -0.0001040 & -0.0000450 & -0.0000024 & -0.0000410 \\
\hline $\begin{array}{l}\mathrm{FEV}_{1} \% \\
\text { predicted }\end{array}$ & -0.0003490 & 0.0000000 & -0.0000003 & 0.0000043 & 0.0000065 & 0.0000535 & 0.0000272 & -0.0000120 & -0.0000006 & -0.0000070 & -0.0000048 & -0.0000160 & 0.0000608 & -0.0000250 & 0.0000395 & 0.0000130 & 0.0000006 & 0.0000079 \\
\hline $\begin{array}{l}\text { Dyspnea } \\
\text { (most days } \\
\text { vs none) } \\
\text { Dyspnea }\end{array}$ & -0.0258700 & 0.0002090 & -0.0000600 & -0.0000680 & 0.0000535 & 0.0334900 & 0.0293000 & -0.0018130 & -0.0000079 & -0.0001500 & -0.0006360 & -0.0000410 & -0.0002130 & 0.0002069 & 0.0005667 & -0.0006370 & -0.0000690 & -0.0000410 \\
\hline $\begin{array}{l}\text { (several } \\
\text { days vs } \\
\text { none) }\end{array}$ & -0.0284300 & 0.0001219 & -0.0000330 & -0.0000300 & 0.0000272 & 0.0293000 & 0.0315300 & -0.0002450 & 0.0000066 & -0.0001020 & -0.0002450 & -0.0004120 & -0.0001360 & 0.0002080 & 0.0004469 & -0.0002120 & -0.0000190 & -0.0002860 \\
\hline $\begin{array}{l}\text { Cough and } \\
\text { sputum }\end{array}$ & 0.0008488 & 0.0000162 & -0.0000140 & -0.0000140 & -0.0000120 & -0.0018130 & -0.0002450 & 0.0057060 & -0.0000078 & 0.0000029 & -0.0002490 & -0.0011720 & 0.0001823 & 0.0000673 & -0.0001740 & 0.0003901 & -0.0000250 & -0.0000420 \\
\hline Age & -0.0012750 & 0.0000011 & -0.0000010 & -0.0000130 & -0.0000006 & -0.0000079 & 0.0000066 & -0.0000078 & 0.0000195 & -0.0000280 & -0.0000300 & 0.0000911 & 0.0000331 & 0.0000290 & 0.0000002 & -0.0000190 & 0.0000010 & 0.0000095 \\
\hline $\begin{array}{l}\text { CVD co- } \\
\text { morbidity }\end{array}$ & 0.0008523 & 0.0000050 & 0.0000020 & 0.0000004 & -0.0000070 & -0.0001500 & -0.0001020 & 0.0000029 & -0.0000280 & 0.0071900 & 0.0001539 & 0.0001192 & 0.0000166 & 0.0002332 & 0.0000239 & -0.0001690 & -0.0000053 & 0.0001453 \\
\hline $\begin{array}{l}\text { Other co- } \\
\text { morbidity }\end{array}$ & -0.0049280 & -0.0000030 & -0.0000960 & -0.0000210 & -0.0000048 & -0.0006360 & -0.0002450 & -0.0002490 & -0.0000300 & 0.0001539 & 0.0098630 & 0.0004256 & -0.0007850 & -0.0004940 & 0.0005407 & 0.0001252 & -0.0000074 & 0.0002297 \\
\hline $\begin{array}{l}\text { Current } \\
\text { smoker }\end{array}$ & -0.0068270 & -0.0000240 & 0.0000161 & -0.0000250 & -0.0000160 & -0.0000410 & -0.0004120 & -0.0011720 & 0.0000911 & 0.0001192 & 0.0004256 & 0.0057430 & -0.0004020 & 0.0003934 & -0.0006680 & -0.0000170 & -0.0000038 & 0.0000165 \\
\hline Sex & -0.0058710 & -0.0000110 & -0.0001100 & 0.0002469 & 0.0000608 & -0.0002130 & -0.0001360 & 0.0001823 & 0.0000331 & 0.0000166 & -0.0007850 & -0.0004020 & 0.0062980 & -0.0005500 & -0.0000880 & -0.0000950 & 0.0000070 & -0.0001050 \\
\hline $\begin{array}{l}\text { BMI (high } \\
\text { vs normal) }\end{array}$ & -0.0008890 & -0.0000200 & -0.0000480 & -0.0000260 & -0.0000250 & 0.0002069 & 0.0002080 & 0.0000673 & 0.0000290 & 0.0002332 & -0.0004940 & 0.0003934 & -0.0005500 & 0.0092850 & 0.0016900 & -0.0008750 & -0.0000120 & 0.0001457 \\
\hline $\begin{array}{l}\text { BMI (low vs } \\
\text { normal) }\end{array}$ & -0.0035210 & 0.0000070 & -0.0000110 & -0.0001040 & 0.0000395 & 0.0005667 & 0.0004469 & -0.0001740 & 0.0000002 & 0.0000239 & 0.0005407 & -0.0006680 & -0.0000880 & 0.0016900 & 0.0091780 & 0.0000211 & -0.0000071 & -0.0002710 \\
\hline mMRC & -0.0122200 & 0.0000035 & 0.0000269 & -0.0000450 & 0.0000130 & -0.0006370 & -0.0002120 & 0.0003901 & -0.0000190 & -0.0001690 & 0.0001252 & -0.0000170 & -0.0000950 & -0.0008750 & 0.0000211 & 0.0174500 & -0.0000570 & -0.0000620 \\
\hline SGRQ & -0.0002420 & 0.0000002 & -0.0000004 & -0.0000024 & 0.0000006 & -0.0000690 & -0.0000190 & -0.0000250 & 0.0000010 & -0.0000053 & -0.0000074 & -0.0000038 & 0.0000070 & -0.0000120 & -0.0000071 & -0.0000570 & 0.0000056 & -0.0000250 \\
\hline Prior exac. & -0.0031080 & 0.0000018 & -0.0000220 & -0.0000410 & 0.0000079 & -0.0000410 & -0.0002860 & -0.0000420 & 0.0000095 & 0.0001453 & 0.0002297 & 0.0000165 & -0.0001050 & 0.0001457 & -0.0002710 & -0.0000620 & -0.0000250 & 0.0054700 \\
\hline
\end{tabular}

BMI, body-mass index; CVD, cardiovascular disease; FEV ${ }_{1}$, forced expiratory volume in one second; mMRC, modified Medical Research Council; SGRQ, St George's Respiratory Questionnaire 
Table A24 Covariance matrix (outpatient visits)

\begin{tabular}{|c|c|c|c|c|c|c|c|c|c|c|c|c|c|c|c|c|c|c|}
\hline & Intercept & $\begin{array}{c}\text { Time (log } \\
\text { years) }\end{array}$ & $\begin{array}{c}\text { Moderate } \\
\text { exac. count }\end{array}$ & $\begin{array}{c}\text { Severe } \\
\text { exac. count }\end{array}$ & $\begin{array}{l}\mathrm{FEV}_{1} \% \\
\text { predicted }\end{array}$ & $\begin{array}{l}\text { Dyspnea } \\
\text { (most days } \\
\text { vs none) }\end{array}$ & $\begin{array}{c}\text { Dyspnea } \\
\text { (several } \\
\text { days vs } \\
\text { none) }\end{array}$ & $\begin{array}{c}\text { Cough and } \\
\text { sputum }\end{array}$ & Age & $\begin{array}{c}\text { CVD co- } \\
\text { morbidity }\end{array}$ & $\begin{array}{l}\text { Other co- } \\
\text { morbidity }\end{array}$ & Sex & $\begin{array}{l}\text { Current } \\
\text { smoker }\end{array}$ & $\begin{array}{l}\text { BMI (high } \\
\text { vs normal) }\end{array}$ & $\begin{array}{c}\text { BMI (low } \\
\text { vs normal) }\end{array}$ & mMRC & SGRQ & Prior exac. \\
\hline Intercept & 0.3999300 & 0.0005560 & -0.0001510 & 0.0021260 & -0.0008500 & -0.0286500 & -0.0352200 & 0.0020600 & -0.0035210 & 0.0037140 & -0.0065340 & -0.0185300 & -0.0157100 & -0.0041190 & -0.0084200 & -0.0442000 & -0.0006750 & -0.0084160 \\
\hline $\begin{array}{l}\text { Time (log } \\
\text { years) }\end{array}$ & 0.0005560 & 0.0005441 & 0.0000167 & -0.0000520 & -0.0000005 & 0.0006241 & 0.0003688 & 0.0001159 & 0.0000041 & 0.0000054 & -0.0000270 & -0.0000710 & -0.0000250 & -0.0000580 & 0.0000107 & -0.0000350 & -0.0000017 & 0.0000249 \\
\hline $\begin{array}{l}\text { Moderate } \\
\text { exac. count }\end{array}$ & -0.0001510 & 0.0000167 & 0.0001667 & -0.0001630 & -0.0000001 & -0.0002600 & -0.0001170 & -0.0000370 & 0.0000008 & 0.0000396 & -0.0002950 & 0.0000301 & -0.0003440 & -0.0001130 & -0.0000610 & 0.0001228 & -0.0000003 & -0.0000300 \\
\hline $\begin{array}{l}\text { Severe exac. } \\
\text { count }\end{array}$ & 0.0021260 & -0.0000520 & -0.0001630 & 0.0020130 & 0.0000153 & 0.0000843 & -0.0000090 & 0.0000641 & -0.0000390 & -0.0001190 & -0.0000630 & -0.0000470 & 0.0006148 & -0.0001960 & -0.0003260 & -0.0001050 & -0.0000120 & -0.0001990 \\
\hline $\begin{array}{l}\text { FEV }_{1} \% \\
\text { predicted }\end{array}$ & -0.0008500 & -0.0000005 & -0.0000001 & 0.0000153 & 0.0000160 & 0.0001184 & 0.0000544 & -0.0000270 & -0.0000018 & -0.0000250 & -0.0000130 & -0.0000400 & 0.0001419 & -0.0000650 & 0.0001086 & 0.0000320 & 0.0000021 & 0.0000231 \\
\hline $\begin{array}{l}\text { Dyspnea } \\
\text { (most days } \\
\text { vs none) } \\
\text { DDspnea }\end{array}$ & -0.0286500 & 0.0006241 & -0.0002600 & 0.0000843 & 0.0001184 & 0.0489900 & 0.0365600 & -0.0041980 & 0.0000145 & -0.0006840 & -0.0013690 & -0.0004630 & -0.0003190 & 0.0002253 & 0.0016450 & -0.0013330 & -0.0001700 & -0.0003390 \\
\hline $\begin{array}{l}\text { (several } \\
\text { days vs } \\
\text { none) }\end{array}$ & -0.0352200 & 0.0003688 & -0.0001170 & -0.0000090 & 0.0000544 & 0.0365600 & 0.0412100 & -0.0005600 & 0.0000485 & -0.0006020 & -0.0004250 & -0.0007950 & 0.0000053 & 0.0005747 & 0.0011650 & -0.0005580 & -0.0000560 & -0.0007840 \\
\hline $\begin{array}{l}\text { Cough and } \\
\text { sputum }\end{array}$ & 0.0020600 & 0.0001159 & -0.0000370 & 0.0000641 & -0.0000270 & -0.0041980 & -0.0005600 & 0.0147200 & -0.0000200 & -0.0000390 & -0.0005080 & -0.0027150 & 0.0005160 & 0.0003459 & -0.0004750 & 0.0008070 & -0.0000680 & -0.0000700 \\
\hline Age & -0.0035210 & 0.0000041 & 0.0000008 & -0.0000390 & -0.0000018 & 0.0000145 & 0.0000485 & -0.0000200 & 0.0000536 & -0.0000960 & -0.0000850 & 0.0002434 & 0.0001034 & 0.0000884 & -0.0000140 & -0.0000620 & 0.0000026 & 0.0000128 \\
\hline $\begin{array}{l}\text { CVD co- } \\
\text { morbidity }\end{array}$ & 0.0037140 & 0.0000054 & 0.0000396 & -0.0001190 & -0.0000250 & -0.0006840 & -0.0006020 & -0.0000390 & -0.0000960 & 0.0174800 & 0.0007264 & 0.0005326 & -0.0001570 & 0.0004505 & 0.0001809 & 0.0001613 & -0.0000250 & 0.0005167 \\
\hline $\begin{array}{l}\text { Other co- } \\
\text { morbidity }\end{array}$ & -0.0065340 & -0.0000270 & -0.0002950 & -0.0000630 & -0.0000130 & -0.0013690 & -0.0004250 & -0.0005080 & -0.0000850 & 0.0007264 & 0.0185600 & 0.0010220 & -0.0019050 & -0.0014010 & 0.0015620 & 0.0003520 & -0.0000096 & 0.0005798 \\
\hline $\begin{array}{l}\text { Current } \\
\text { smoker }\end{array}$ & -0.0185300 & -0.0000710 & 0.0000301 & -0.0000470 & -0.0000400 & -0.0004630 & -0.0007950 & -0.0027150 & 0.0002434 & 0.0005326 & 0.0010220 & 0.0157300 & -0.0010710 & 0.0010070 & -0.0017430 & 0.0002734 & -0.0000035 & 0.0000644 \\
\hline Sex & -0.0157100 & -0.0000250 & -0.0003440 & 0.0006148 & 0.0001419 & -0.0003190 & 0.0000053 & 0.0005160 & 0.0001034 & -0.0001570 & -0.0019050 & -0.0010710 & 0.0245900 & -0.0015380 & -0.0000900 & -0.0004240 & 0.0000273 & -0.0000270 \\
\hline $\begin{array}{l}\text { BMI (high } \\
\text { vs normal) }\end{array}$ & -0.0041190 & -0.0000580 & -0.0001130 & -0.0001960 & -0.0000650 & 0.0002253 & 0.0005747 & 0.0003459 & 0.0000884 & 0.0004505 & -0.0014010 & 0.0010070 & -0.0015380 & 0.0388200 & 0.0044430 & -0.0019120 & -0.0000280 & 0.0006994 \\
\hline $\begin{array}{l}\text { BMI (low vs } \\
\text { normal) }\end{array}$ & -0.0084200 & 0.0000107 & -0.0000610 & -0.0003260 & 0.0001086 & 0.0016450 & 0.0011650 & -0.0004750 & -0.0000140 & 0.0001809 & 0.0015620 & -0.0017430 & -0.0000900 & 0.0044430 & 0.0184500 & 0.0000636 & -0.0000290 & -0.0007420 \\
\hline mMRC & -0.0442000 & -0.0000350 & 0.0001228 & -0.0001050 & 0.0000320 & -0.0013330 & -0.0005580 & 0.0008070 & -0.0000620 & 0.0001613 & 0.0003520 & 0.0002734 & -0.0004240 & -0.0019120 & 0.0000636 & 0.0587500 & -0.0001590 & -0.0003410 \\
\hline SGRQ & -0.0006750 & -0.0000017 & -0.0000003 & -0.0000120 & 0.0000021 & -0.0001700 & -0.0000560 & -0.0000680 & 0.0000026 & -0.0000250 & -0.0000096 & -0.0000035 & 0.0000273 & -0.0000280 & -0.0000290 & -0.0001590 & 0.0000142 & -0.0000600 \\
\hline Prior exac. & -0.0084160 & 0.0000249 & -0.0000300 & -0.0001999 & 0.0000231 & -0.0003390 & -0.0007840 & -0.0000700 & 0.0000128 & 0.0005167 & 0.0005798 & 0.0000644 & -0.0000270 & 0.0006994 & -0.0007420 & -0.0003410 & -0.0000600 & 0.0151700 \\
\hline
\end{tabular}

BMI, body-mass index; CVD, cardiovascular disease; FEV ${ }_{1}$, forced expiratory volume in one second; mMRC, modified Medical Research Council; SGRQ, St George's Respiratory Questionnaire 